\title{
INDISCRIMINATE COVERS OF INFINITE TRANSLATION SURFACES ARE INNOCENT, NOT DEVIOUS
}

\author{
W. PATRICK HOOPER AND RODRIGO TREVIÑO
}

The private lives of surfaces
are innocent, not devious.
- Kay Ryan

\begin{abstract}
We consider the interaction between passing to finite covers and ergodic properties of the straight-line flow on finite area translation surfaces with infinite topological type. Infinite type provides for a rich family of degree $d$ covers for any integer $d>1$. We give examples which demonstrate that passing to a finite cover can destroy ergodicity, but we also provide evidence that this phenomenon is rare. We define a natural notion of a random degree $d$ cover and show that, in many cases, ergodicity and unique ergodicity are preserved under passing to random covers. This work provides a new context for exploring the relationship between recurrence of the Teichmüller flow and ergodic properties of the straight-line flow.
\end{abstract}

\section{INTRODUCTION}

A translation surface is a pair $(S, \alpha)$, where $S$ is a Riemann surface and $\alpha$ is a holomorphic 1 -form on $S$. Let $Z \subset S$ denote the zeros of $\alpha$. The 1 -form $\alpha$ endows $S \backslash Z$ with local coordinates to the plane: for any $p$ we have the locally defined coordinate chart to $\mathbb{C}$ given by the local homeomorphism $q \mapsto \int_{p}^{q} \alpha$. These coordinate charts differ locally only by translation. A translation surface inherits a metric by pulling back the Euclidean metric on the plane along the coordinate charts. Points in $S \backslash Z$ are locally isometric to the plane, while points in $Z$ are cone singularities with cone angle $2(k+1) \pi$ where $k \geq 1$ is the degree of the zero of $\alpha$.

We'll say a translation surface is classical if $S$ is a closed surface. Here, there is a well known interplay between two types of dynamical systems: (1) the dynamics of the (horizontal) translation flow on a translation surface $(S, \alpha)$ given in local coordinates by

$$
F^{t}: S \rightarrow S ; \quad(x, y) \mapsto(x+t, y),
$$

and (2) the Teichmüller deformation $g^{t}$ on the moduli space of translation surfaces, where $g^{t}(S, \alpha)$ is obtained from $(S, \alpha)$ by postcomposing the coordinate charts with the affine coordinate change $g^{t}(x, y)=\left(e^{-t} x, e^{t} y\right)$. Namely, the Teichmüller deformation renormalizes the translation flow. A famous consequence of this relationship is given by Masur's Criterion: if the forward orbit of the Teichmüller deformation, $\left\{g^{t}(S, \alpha)\right\}_{t \geq 0}$, has a convergent subsequence $g^{t_{n}}(S, \alpha)$ with $t_{n} \rightarrow \infty$ (i.e., the orbit is non-divergent), then the translation flow is uniquely ergodic [Mas92].

A surface has infinite topological type if its fundamental group is not finitely generated. This includes infinite genus surfaces. Recently, many results similar in spirit to Masur's 
criterion have been proven in special cases for translation surfaces of infinite topological type [Hoo15, HW13, Tre14, Tre16].

Reasoning about infinite type translation surfaces introduces several difficulties:

(1) Translation flow trajectories on a infinite type surface may not be defined for all time. In fact, there are interesting examples where trajectories are defined nowhere for some fixed time, for example the Icicled surface of Randecker [Ran14, Example 5.5]. In order to consider ergodicity, we require the translation flow to be defined for all time almost everywhere (i.e., for all $t$, the time $t$ map $F^{t}$ is defined a.e.).

(2) While the translation flow is continuous, the domain is not compact.

(3) There is no well established moduli space of infinite-type translation surfaces. (In this direction, the preprints [Hoo16] and [Hoo13] propose a topology for the space of translation surfaces with basepoint.)

In the classical case (1) and (2) can be easily resolved (via standard coding arguments) and moduli space is a finite dimension orbifold. The translation flow can often be easily shown to be defined for all time almost everywhere, and we will in fact be assuming stronger hypotheses. Difficulty (2) simply must be worked around. A standard approach to work around (3) involves defining some topological space of surfaces where $S L(2, \mathbb{R})$ acts and prove that non-divergence of an orbit $t \mapsto g^{t}(S, \alpha)$ within this space entails consequences for the translation flow on $(S, \alpha)$. To date, the primary mechanism for building such a topological space of surfaces uses affine symmetries of the translation surface $(S, \alpha)$, as we will now explain.

Two translation surfaces $(S, \alpha)$ and $\left(S^{\prime}, \alpha^{\prime}\right)$ are translation equivalent if there is a homeomorphism $h: S \rightarrow S^{\prime}$ which is a translation in local coordinates. Let $S L_{ \pm}(2, \mathbb{R})$ denote the group of $2 \times 2$ real matrices with determinant \pm 1 . The group $S L_{ \pm}(2, \mathbb{R})$ acts on the collection of all translation surfaces by simultaneous postcomposition with all local charts; see 3 . We define $\mathcal{O}(S, \alpha)$ to be the $S L_{ \pm}(2, \mathbb{R})$ orbit:

$$
\mathcal{O}(S, \alpha)=\left\{A(S, \alpha): A \in S L_{ \pm}(2, \mathbb{R})\right\} / \text { translation equivalence. }
$$

The orbit $\mathcal{O}(S, \alpha)$ is parameterized by a choice of $A \in S L_{ \pm}(2, \mathbb{R})$ and thus inherits a topology as a topological quotient space. This space can be described concretely as the quotient of $S L_{ \pm}(2, \mathbb{R})$ by a subgroup, namely the surface's Veech group,

$$
V(S, \alpha)=\left\{A \in S L_{ \pm}(2, \mathbb{R}): A(S, \alpha) \text { is translation equivalent to }(S, \alpha)\right\} .
$$

Observe that two surfaces $A_{1}(S, \alpha)$ and $A_{2}(S, \alpha)$ with $A_{1}, A_{2} \in S L_{ \pm}(2, \mathbb{R})$ are translation equivalent if and only if $A_{1}$ and $A_{2}$ determine the same left coset (or equivalently, if $\left.A_{2}^{-1} A_{1} \in V(S, \alpha)\right)$. Thus, there is a natural identification between $\mathcal{O}(S, \alpha)$ and the left coset space $S L_{ \pm}(2, \mathbb{R}) / V(S, \alpha)$. In particular, this structure allows us to say that the Teichmüller trajectory $g^{t}(S, \alpha)$ is non-divergent in $\mathcal{O}(S, \alpha)$ if there is a sequence $t_{n} \rightarrow+\infty$ so that $g^{t_{n}}(S, \alpha)$ converges in $\mathcal{O}(S, \alpha)$.

Work of the second author [Tre14, Theorem 2] (in this paper as Corollary 20) shows that if a finite area translation surface of possibly infinite genus $(S, \alpha)$ has a non-divergent Teichmüller trajectory in $\mathcal{O}(S, \alpha)$, then the translation flow is ergodic on $(S, \alpha)$. It seems reasonable to conjecture that in fact unique ergodicity holds under these hypotheses: Separate works of the two co-authors each prove results to this effect in special cases; see Example F.2 and Appendix H of [Hoo15] and the main result of [Tre16]. 
Suppose $(S, \alpha)$ is a finite area translation surface with infinite topological type which has a translation flow which is defined for all time almost everywhere and is ergodic. Any cover of $S$ inherits a translation structure by pulling back the 1-form $\alpha$ under the covering map. When endowed with this structure, the cover's translation flow will also be defined for all time almost everywhere. In this paper we study the ergodicity of the translation flow on finite unbranched covers $(\tilde{S}, \tilde{\alpha})$ of $(S, \alpha)$. We introduce some spaces of covers and following the above paradigm study how the behavior of Teichmüller trajectories through this space influences the ergodicity of the translation flow on a cover.

In order to say something constructive, we pick some integer $d \geq 2$, and restrict attention to covers of degree $d$. Choose an arbitrary non-singular basepoint on the surface $S$. Let $(\tilde{S}, \tilde{\alpha})$ be a cover of $(S, \alpha)$, where the flat structure on $(\tilde{S}, \tilde{\alpha})$ is lifted from the one on $(S, \alpha)$. The fiber of the basepoint can be identified in an arbitrary way with the set $\{1,2, \ldots, d\}$. The monodromy action is the natural right action of the fundamental group of $S$ on the fiber of the basepoint. Our identification of the fiber determines a monodromy representation $\pi_{1}(S) \rightarrow \Pi_{d}$, where $\Pi_{d}$ is the permutation group of $\{1, \ldots, d\}$. We note that a cover can be reconstructed from its monodromy representation, and two covers are isomorphic (in the sense of covering theory) if and only if these monodromy representations differ by conjugation by an element of $\Pi_{d}$. These ideas are reviewed in $\$ 4.1$. Now fix a subgroup $G \subset \Pi_{d}$. We say a cover $(\tilde{S}, \tilde{\alpha})$ has monodromy in $G$ if it can be realized by a monodromy representation to $G$. From the above remarks, we note that the space of covers of $(S, \alpha)$ with monodromy in $G$ up to cover isomorphism is identified with

$$
\Pi_{d} \backslash \operatorname{Hom}\left(\pi_{1}(S), G\right),
$$

where $\Pi_{d}$ acts on $\operatorname{Hom}\left(\pi_{1}(S), G\right)$ by conjugation. We endow $\operatorname{Hom}\left(\pi_{1}(S), G\right)$ with the product topology by viewing it as a subset of $G^{\pi_{1}(S)}$, where the finite set $G$ is given the discrete topology. In particular, $\operatorname{Hom}\left(\pi_{1}(S), G\right)$ is homeomorphic to a Cantor set, since $\pi_{1}(S)$ is a free group with countably many generators; see $\$ 4.1$.

We define $\operatorname{Cov}_{G}(S, \alpha)$ to be the collection of all covers of $(S, \alpha)$ with monodromy in $G$ up to translation equivalence. Translation equivalence is a coarser notion of equivalence than covering isomorphism, and we give $\operatorname{Cov}_{G}(S, \alpha)$ the quotient topology by viewing this space of covers as a topological quotient of $\Pi_{d} \backslash \operatorname{Hom}\left(\pi_{1}(S), G\right)$. The choice of $h \in \operatorname{Hom}\left(\pi_{1}(S), G\right)$ determines a cover $\left(\tilde{S}_{h}, \tilde{\alpha}_{h}\right)$ with $h$ describing the monodromy of the cover. We note that the cover need not be connected. In fact $\left(\tilde{S}_{h}, \tilde{\alpha}_{h}\right)$ is connected if and only if the image of the monodromy representation, $h\left(\pi_{1}(S)\right)$ acts transitively on $\{1, \ldots, d\}$.

The main object of interest to this paper is the union of $S L_{ \pm}(2, \mathbb{R})$ orbits of covers of $(S, \alpha)$ with monodromy in $G$ :

$$
\tilde{\mathcal{O}}_{G}(S, \alpha)=\left\{A(\tilde{S}, \tilde{\alpha}): A \in S L_{ \pm}(2, \mathbb{R}) \text { and }(\tilde{S}, \tilde{\alpha}) \in \operatorname{Cov}_{G}(S, \alpha)\right\} / \sim,
$$

where $\sim$ denotes translation equivalence. We call the action of the diagonal subgroup $g^{t}$ of $S L_{ \pm}(2, \mathbb{R})$ on $\tilde{\mathcal{O}}_{G}(S, \alpha)$ the cover cocycle. Again, $\tilde{\mathcal{O}}_{G}(S, \alpha)$ inherits a topology because it can be considered a topological quotient of $S L_{ \pm}(2, \mathbb{R}) \times \operatorname{Cov}_{G}(S, \alpha)$. This is a natural space in which to study Teichmüller deformations of covers of $(S, \alpha)$. Like $\operatorname{Cov}_{G}(S, \alpha)$, this space contains both connected and disconnected surfaces provided $G$ acts transitively on $\{1, \ldots, d\}$. We prove the following:

Theorem 1 (Connected accumulation point implies ergodicity). Let $d \geq 2$ be an integer and let $G \subset \Pi_{d}$ be a subgroup which acts transitively on $\{1, \ldots, d\}$. Let $(S, \alpha)$ be a finite area 
translation surface with infinite topological type, and let $(\tilde{S}, \tilde{\alpha})$ be a cover with monodromy in $G$. Then, the translation flow on $(\tilde{S}, \tilde{\alpha})$ is defined for all time almost everywhere and is ergodic if the Teichmüller trajectory $g^{t}(\tilde{S}, \tilde{\alpha})$ has an $\omega$-limit point in $\tilde{\mathcal{O}}_{G}(S, \alpha)$ representing a connected surface.

Theorem 1 is proved in $\$ 5$. We note that ergodicity of the translation flow on a finite cover has consequences for classifying the invariant measures.

Proposition 2 (Ergodicity and unique lifts of measures). Suppose that $(\tilde{S}, \tilde{\alpha})$ is a finite cover of the finite area translation surface $(S, \alpha)$ with infinite topological type. Also suppose that both surfaces have translation flows which are defined for all time almost everywhere and are ergodic. Then, Lebesgue measure on $(\tilde{S}, \tilde{\alpha})$ is the unique translation flow-invariant measure which projects to Lebesgue measure on $(S, \alpha)$ under the covering map.

See $\$ 6$ for the brief proof.

Corollary 3 (Lifting unique ergodicity). Suppose the conditions of Proposition 2 are satisfied and additionally the translation flow on $(S, \alpha)$ is uniquely ergodic. Then, the translation flow on the cover $(\tilde{S}, \tilde{\alpha})$ is uniquely ergodic.

Proof. Suppose $\mu$ is a translation flow-invariant probability measure on $(\tilde{S}, \tilde{\alpha})$. Then its push forward under the covering map, $p_{*} \mu$ is a translation-flow invariant measure on $(S, \alpha)$, and by uniqueness is necessarily Lebesgue measure. The proposition guarantees that $\mu$ is also Lebesgue measure.

The existence of a Veech group for the surface $(S, \alpha)$ gives a mechanism for generating the type of accumulation we need for applying Theorem 1 .

Proposition 4. Suppose $(S, \alpha)$ is a finite area translation surface with infinite topological type, and let $(\tilde{S}, \tilde{\alpha})$ be a cover with monodromy in $G$. If $(S, \alpha)$ has a non-divergent Teichmüller trajectory in $\mathcal{O}(S, \alpha)$, then the Teichmüller trajectory of the cover $g^{t}(\tilde{S}, \tilde{\alpha})$ has an $\omega$-limit point in $\tilde{\mathcal{O}}_{G}(S, \alpha)$.

Again, see $\$ 6$ for the proof. In order to use the conclusions of Theorem 1, we would need to know that there is a connected $\omega$-limit point. We expect that it is difficult in general to determine precisely when this is true. However, we will introduce a notion under which most covers have connected accumulation points.

Let $G \subset \Pi_{d}$ be as above, and let $(S, \alpha)$ be a finite area translation surface with infinite topological type. We explain in $\$ 4.2$ that there is a natural Borel probability measure $m_{G}$ on the space $\operatorname{Cov}_{G}(S, \alpha)$ of covers with monodromy in $G$ which is invariant under automorphisms of the base surface $S$. This gives us a notion of a "random cover." Informally, the measure $m_{G}$ corresponds to the notion of random cover obtained by flipping a fair coin to determine the images of the generators of the fundamental group under the monodromy representation.

Theorem 5 (Random covers accumulate on connected covers). Let $(S, \alpha)$ be a finite area translation surface with infinite topological type, and suppose that it has a non-divergent Teichmüller trajectory in $\mathcal{O}(S, \alpha)$. Suppose $G$ is a transitive subgroup of the permutation group $\Pi_{d}$. Then $m_{G}$-almost every cover $(\tilde{S}, \tilde{\alpha}) \in \operatorname{Cov}_{G}(S, \alpha)$ has a Teichmüller trajectory with a connected accumulation point.

The proof lies in $\$ 6$. As a consequence of Theorem 1, we see: 
Corollary 6 (Ergodicity of random covers). With the hypotheses of Theorem 5, $m_{G}$-almost every cover of $(S, \alpha)$ with monodromy in $G$ has ergodic translation flow.

Corollary 6 has applications to certain types of finite skew product extensions of $n$-adic odometers as follows. For any integer $n \geq 2$, let $X_{n}=\{0, \ldots, n-1\}^{\mathbb{N}}$ be given the product topology. The $n$-adic odometer is the map $\Omega_{n}: X_{n} \rightarrow X_{n}$ defined as addition by 1 with infinite carry to the right:

$$
\Omega_{n}: x=\left(x_{1}, x_{2}, \ldots\right) \mapsto\left(0,0, \ldots, x_{\kappa(x)}+1, x_{\kappa(x)+1}, x_{\kappa(x)+2}, \ldots\right),
$$

where $\kappa(x) \in \mathbb{N} \cup\{+\infty\}$ is the smallest index $k \in \mathbb{N}$ so that $x_{k} \neq n-1$ or $+\infty$ if no such index exists.

Denote by $\Gamma^{+}$the free group on the countably infinite set of generators $\left\{\gamma_{i}: i \in \mathbb{N}\right\}$. Let $n \geq 2$ be an integer and $G$ a subgroup of $\Pi_{d}$. For any $\psi_{+} \in \operatorname{Hom}\left(\Gamma^{+}, G\right)$, we define the skew product over the $n$-adic odometer $E_{\psi_{+}}: X_{n} \times\{1, \ldots, d\} \rightarrow X_{n} \times\{1, \ldots, d\}$ as

$$
E_{\psi_{+}}(x, m)=\left(\Omega_{n}(x), \psi_{+}\left(\gamma_{\kappa(x)}\right)(m)\right),
$$

where $\kappa(x)$ is as above. The image $E_{\psi_{+}}(x, m)$ is well defined unless $\kappa(x)=+\infty$, but we ignore this issue because points have well defined orbits off a countable set.

The space $\operatorname{Hom}\left(\Gamma^{+}, G\right)$ parameterizes these skew products and comes with a natural product measure as in Definition 12 in $\$ 4.2$. We will denote this measure by $\mu_{+}$.

Theorem 7. Let $n \geq 2$ be an integer and $G$ be a subgroup of $\Pi_{d}$ that acts transitively on $\{1, \ldots, d\}$. Then for $\mu_{+}$-almost every $\psi_{+} \in \operatorname{Hom}\left(\Gamma^{+}, G\right)$, the skew product $E_{\psi_{+}}$is uniquely ergodic.

The connection between translation flows and $n$-adic odometers comes from the fact that the suspension flow over an $n$-adic odometer is measurably isomorphic to the translation flow on the infinite genus surface first studied by Chamanara. The skew-products described here have a suspension given by translation flow on a cover of Chamanara's surface. As such, the theorem follows as a consequence of Corollaries 3 and 6 . We prove this result at the beginning of $\$ 7.1$.

Devious covers. The above discussion has left open a natural question: What happens if $g^{t}(S, \alpha)$ is non-divergent in $\mathcal{O}(S, \alpha)$, but the Teichmüller orbit of the connected cover $(\tilde{S}, \tilde{\alpha})$ only accumulates on disconnected covers in $\tilde{\mathcal{O}}_{G}(S, \alpha)$. We call such covers devious.

Apparently there is no a priori reason why the translation flow on a devious cover should be ergodic or non-ergodic. We illustrate this by example in $\$ 7$.

In $\$ 7.1$ after proving Theorem 7 , we concentrate on devious covers of the surface $\left(S_{2}, \alpha_{2}\right)$ of Chamanara which is related to the 2 -adic odometer. Devious $G$-covers with non-ergodic translation flow occur for all $G$ for fairly trivial reasons in this case. (The surface $\left(S_{2}, \alpha_{2}\right)$ has horizontal saddle connections which can connect the surface but are not seen by the translation flow.) However we also exhibit devious covers of $\left(S_{2}, \alpha_{2}\right)$ whose translation flow is uniquely ergodic. Our construction of such covers works as long as the subgroup $G \subset \Pi_{d}$ is large enough to contain two subgroups $H_{1}$ and $H_{2}$ which fail to act transitively on $\{1, \ldots, d\}$ but so that $H_{1} \cup H_{2}$ generates a subgroup of $G$ which does act transitively.

The translation flow on Chamanara's surface is special for many reasons, including that the Teichmüller trajectory of this surface is periodic in $\mathcal{O}\left(S_{2}, \alpha_{2}\right)$, and so we consider a different surface in $\$ 7.2$. We consider double covers of affine images of a translation surface we call the ladder surface, $\left(S_{L}, \alpha_{L}\right)$. (See Figure 4 on page 32.) The ladder surface has a 
non-elementary Veech group containing a parabolic and a reflection. We produce several possible behaviors of the translation flow on double covers of $A\left(S_{L}, \alpha_{L}\right)$ :

(L1) For some $A \in S L_{ \pm}(2, \mathbb{R})$, there are no devious double covers of $A\left(S_{L}, \alpha_{L}\right)$, i.e. for every connected double cover $(\tilde{S}, \tilde{\alpha})$, the forward orbit $g^{t} A(\tilde{S}, \tilde{\alpha})$ accumulates on a connected cover in $\tilde{\mathcal{O}}_{\mathbb{Z}_{2}}\left(S_{L}, \alpha_{L}\right)$ so that $A(\tilde{S}, \tilde{\alpha})$ always has ergodic translation flow.

(L2) For some $A \in S L_{ \pm}(2, \mathbb{R})$, there are devious double covers of $A\left(S_{L}, \alpha_{L}\right)$ but nonetheless every connected double cover has ergodic translation flow.

(L3) For some $A \in S L_{ \pm}(2, \mathbb{R})$, there are devious double covers of $A\left(S_{L}, \alpha_{L}\right)$ and every devious double cover has non-ergodic translation flow.

Let us briefly summarize how to distinguish the cases above. We use $V^{\prime} \subset S L_{ \pm}(2, \mathbb{R})$ to denote the known Veech group of $\left(S_{L}, \alpha_{L}\right)$. In all the cases we assume that $g^{t} A V^{\prime}$ is nondivergent in $S L_{ \pm}(2, \mathbb{R}) / V^{\prime}$, which implies that $g^{t} A\left(S_{L}, \alpha_{L}\right)$ is non-divergent in $\mathcal{O}\left(S_{L}, \alpha_{L}\right)$. We find an infinite index subgroup $\tilde{V}^{\prime} \subset V^{\prime}$ with the property that $\tilde{V}^{\prime} \subset V(\tilde{S}, \tilde{\alpha})$ for every double cover $(\tilde{S}, \tilde{\alpha})$ of $\left(S_{L}, \alpha_{L}\right)$. We are in case $(\mathrm{L} 1)$ if $g^{t} A \tilde{V}^{\prime}$ is non-divergent in $S L_{ \pm}(2, \mathbb{R}) / \tilde{V}^{\prime}$. (Statement (L1) follows from Proposition 38 of $\$ 7.2$.) Now assume $g^{t} A \tilde{V}^{\prime}$ is divergent. We describe in Theorem 42 a combinatorial measurement $v(A)$ of the divergence rate of the trajectory $g^{t} A \tilde{V}^{\prime}$ in $S L_{ \pm}(2, \mathbb{R}) / \tilde{V}^{\prime}$. As $v(A)$ decreases the trajectory diverges quicker. We show that if $v(A)>\varphi^{2}$ where $\varphi$ is the golden mean then we are in case (L2). On the other hand if $v(A)<\varphi^{2}$, then we are in case (L3). (Statements (L2) and (L3) appear again in Theorem 42,) The sharpness of this transition demonstrates the power of the methods used to distinguish ergodic and non-ergodic behavior. We wonder:

(L2 $\frac{1}{2}$ ) Could there be an $A \in S L_{ \pm}\left(2, \mathbb{R}\right.$ ) (with $v(A)=\varphi^{2}$ ) so that some devious double covers of $\left(S_{L}, \alpha_{L}\right)$ exhibit ergodic translation flow while others exhibit non-ergodic translation flow?

Acknowledgments. The authors would like thank the anonymous referee for numerous useful comments which greatly improved the exposition. This project began at ICERM which provided a stimulating environment. W. P. H. was supported by N.S.F. Grants DMS1101233 and DMS-1101233 as well as by a PSC-CUNY Award (funded by The Professional Staff Congress and The City University of New York). R. T. was supported by BSF grant 2010428, ERC starting grant DLGAPS 279893, NSF Postdoctoral Fellowship DMS-1204008, and an AMS-Simons Travel Grant. The quote from on the first page is taken from Surfaces in Rya97.

\section{Context on Finite AREA Flat surfaces of Infinite topologicAl TyPe}

There has been an increased interest in the study of the dynamics and geometry of flat surfaces of infinite genus. Unlike classical flat surfaces (which are compact flat surfaces of finite type), there is no natural space for parametrizing flat metrics for all surfaces of a given topological type. This gives the first obstacle to utilizing tools from the theory of compact surfaces. Different techniques have been developed to overcome this fundamental shortcoming, which prevents us from developing a theory to answer one of the most basic questions: whether the translation flow on a given flat surface of infinite type and finite area is ergodic or not.

Most studies concentrate on the case of the surface having finite or infinite area (a notably exception being [Hoo15], where the methods work for surfaces of finite and infinite area). Such choice has great implications to the tools used, the results obtained, and the method 
of construction used to produce examples or to define some "spaces of surfaces". A common tool in both contexts is the use of Veech groups, which are a sort of symmetry groups of the surface. For compact flat surfaces, these are always discrete subgroups of $S L(2, \mathbb{R})$. Flat surfaces of finite type with non-trivial Veech groups are part of a very deep theory which has grown out of the foundational contributions of Thurston [Thu88] and Veech [Vee89], so the fact that they can be used in the infinite type setting is encouraging.

We will concentrate here on the development of the theory of flat surfaces of infinite type and finite area, though there is also a rapidly developing theory of flat surfaces of infinite type and infinite area (where often interest centers on infinite abelian covers of finite type flat surfaces).

To our knowledge, the first papers on dynamics on flat surfaces of infinite type are those which come out of the infinite step polygonal billiards introduced in [Tro99, DEDML98] through the unfolding procedure. All such surfaces considered were of finite area and came from "rational" polygons, i.e., the angles of the billiard from which the surfaces were constructed satisfied some rationality conditions. Ergodic properties as well as topological results were obtained for a large class of these types of surfaces. The approach of these articles is very different from the approach here, because arguments do not make use of Veech groups.

The seminal paper of Chamanara Cha04 introduced a 1-parameter family of flat surfaces of infinite type and of finite area with a non-trivial Veech group. The main results of that paper discussed the Veech groups that appear. Most importantly, even though the surfaces constructed possess many symmetries, the Veech group is never a lattice for any surface arising in this construction. We review Chamanara's construction in section 7 and apply some of our results to spaces of covers thereof.

Another study of flat surfaces of infinite type and finite area has been the construction of Bowman which extends the Arnoux-Yoccoz family of flat surfaces to include a surface of infinite genus and finite area [Bow13]. This surface of infinite genus and finite area admits a affine automorphism with hyperbolic derivative. Moreover, it was found that the Veech group of this surface is isomorphic to $\mathbb{Z} \times \mathbb{Z}_{2}$ and that the directions preserved by the hyperbolic automorphism correspond to uniquely ergodic translation flows.

In [Hoo15, a construction of Thurston is modified to produce infinite genus flat surfaces with non-elementary Veech groups. The construction sometimes produces finite area surfaces, and in this case the translation flow can be shown to be uniquely ergodic on various affine images of the surfaces.

The work [Tre14] addresses the question of ergodicity of translation flows for surfaces of finite area. The hypotheses of the main results are independent of topological type and therefore can be used to determine when the translation flow of a flat surface of infinite genus and finite area is ergodic or uniquely ergodic. We use this criterion in section 5 to study ergodic properties of translation flows on covers of infinite translation surfaces.

Finally, in [LTn16] a way of constructing flat surfaces of finite area and infinite genus is developed through a connection to adic and cutting and stacking transformations, generalizing constructions of Bufetov in the classical case [Buf13]. Among other things, it is shown there that translation flows on surfaces of infinite genus and finite area can exhibit behavior which does not occur for translation flows on compact flat surfaces.

In general it is not well understood what Veech groups can arise for a flat surface of infinite type and finite area. In particular, it is unknown whether there exists a flat surface of infinite type and finite area whose Veech group is a lattice in $S L_{ \pm}(2, \mathbb{R})$. (In contrast, it is known 
that for practically any subgroup $G$ of $S L_{ \pm}(2, \mathbb{R})$, there is a flat surface of infinite type and infinite area whose Veech group is $G$ PSV11.)

As it can be seen, Veech groups have played a significant role in the study of translation flows. The properties of translation flows so far obtained for surfaces of infinite genus and finite area are mostly similar to those of compact flat surfaces.

In this paper, we broaden the scope of known phenomena of translation flows on flat surfaces by utilizing the structure provided by coverings to produce spaces of flat surfaces of infinite type and finite area which are invariant under Teichmüller deformations, and provide sufficiently interesting Teichmüller dynamics so that varied phenomena appear for corresponding translation flows. This construction allows us to determine when the lift of a translation flow to a cover retains ergodic properties which the base surface possesses, such as unique ergodicity. This approach thus overcomes some of the problems created by not having a natural parameter space for infinite type flat surfaces.

\section{BACKGROUND ON TRANSLATION SURFACES}

Let $(S, \alpha)$ be a translation surface and let $Z \subset S$ denote the zeros of $\alpha$. The straight line flow on $(S, \alpha)$ in direction $\theta$ is the flow $F_{\theta}^{t}: S \rightarrow S$ defined for $t \in \mathbb{R}$ given in local coordinates by $F_{\theta}^{t}(x, y)=(x+t \cos \theta, y+t \sin \theta)$ away from the zeros of $\alpha$. We reserve the name translation flow for the straight line flow $F_{\theta}^{t}$ where $\theta=0$. This is the flow on $S$ determined by the rightward unit vector field $X$. We will use $Y$ denote the upward unit vector field.

A more global definition of the straight line flows can be done as follows. Since $\alpha$ is holomorphic, the 1-forms $\Re(\alpha)$ and $\Im(\alpha)$ are harmonic, and thus closed. Therefore, the distributions $\operatorname{ker} \Im(\alpha)$ and $\operatorname{ker} \Re(\alpha)$ define a pair of foliations away from $Z$, the horizontal and vertical foliations. The generators of the distributions in the unit tangent bundle of $S-Z$ are the vector fields $X_{\alpha}$ and $Y_{\alpha}$ which generate, respectively, the horizontal and vertical flows. The translation flow, as defined above, then corresponds to the horizontal flow. From this point of view, we will denote by $\varphi_{t}^{X_{\alpha}}$ and $\varphi_{t}^{Y_{\alpha}}$, respectively, the horizontal and vertical flows.

There is a group of deformations of the flat metric on $(S, \alpha)$ which is parametrized by the group $G L(2, \mathbb{R})$. We will mostly be interested in the action of the area preserving subgroup $S L_{ \pm}(2, \mathbb{R}) \subset G L(2, \mathbb{R})$, and this is the $S L_{ \pm}(2, \mathbb{R})$ action mentioned in the introduction. Fix a matrix $A \in G L(2, \mathbb{R})$. We get new (non-conformal) local coordinate charts to the plane by postcomposing the charts on $(S, \alpha)$ to $\mathbb{C}$ by the real-linear map

$$
A: \mathbb{C} \rightarrow \mathbb{C} ; \quad x+i y \mapsto a_{1,1} x+a_{1,2} y+i\left(a_{2,1} x+a_{2,2} y\right) .
$$

Then, we get a new Riemann surface structure $S^{\prime}$ on $S$ by pulling back the complex structure using these deformed charts, and the charts determine a new holomorphic 1-form $\alpha^{\prime}$ on $S^{\prime}$. We define $A(S, \alpha)=\left(S^{\prime}, \alpha^{\prime}\right)$. The action of the rotation subgroup $S O(2, \mathbb{R})$ parametrizes the directional flows on a given surface $(S, \alpha)$ : for $\theta \in S O(2, \mathbb{R}) \simeq S^{1}$, the horizontal flow on $\theta(S, \alpha)$ corresponds to the straight line flow on $(S, \alpha)$ in direction $\theta$.

Let $(S, \alpha)$ and $\left(S^{\prime}, \alpha^{\prime}\right)$ be translation surfaces. We say they are translation equivalent if there is a homeomorphism $h: S \rightarrow S^{\prime}$ which is a locally a translation in local coordinate charts provided by the 1 -forms. The Veech group of $(S, \alpha)$ is the $\operatorname{subgroup} V(S, \alpha) \subset$ $S L_{ \pm}(2, \mathbb{R})$ of elements $A \in S L_{ \pm}(2, \mathbb{R})$ so that $A(S, \alpha)$ and $(S, \alpha)$ are translation equivalent. An affine homeomorphism from a translation surface $(S, \alpha)$ to another translation surface $\left(S^{\prime}, \alpha^{\prime}\right)$ is a diffeomorphism $\phi: S \rightarrow S^{\prime}$ whose derivative $D(\phi)$ is constant when measured 
using local coordinates provided by the 1-forms. By considering the derivative $D(\phi)$ to be a real linear map we have $D(\phi) \in G L(2, \mathbb{R})$. We can describe this condition in terms of the horizontal and vertical vectors on $(S, \alpha)$ and $\left(S, \alpha^{\prime}\right)$ using the equation

$$
\left(\begin{array}{c}
\phi_{*} X_{\alpha} \\
\phi_{*} Y_{\alpha}
\end{array}\right)=A\left(\begin{array}{c}
X_{\alpha^{\prime}} \\
Y_{\alpha^{\prime}}
\end{array}\right)
$$

where $\phi_{*}$ denotes the push forward action on vector fields and $A=D(\phi)$ is a $2 \times 2$ matrix (the derivative). Observe that the statement that $A(S, \alpha)$ and $\left(S^{\prime}, \alpha^{\prime}\right)$ are translation equivalent is equivalent to the statement that there is a affine homeomorphism $\phi:(S, \alpha) \rightarrow\left(S^{\prime}, \alpha^{\prime}\right)$ with derivative $A$. The affine automorphism group of $(S, \alpha), \operatorname{Aff}(S, \alpha)$ is the group of all affine homeomorphisms from $(S, \alpha)$ to itself. By the prior observation, we have

$$
D(A f f(S, \alpha))=V(S, \alpha) .
$$

\section{Finite COVERS OF infinite SURFACES}

In this section, we work out the theory of spaces of finite degree covers of a translation surface $(S, \alpha)$ of infinite topological type. We describe the topology of the space of covers $\operatorname{Cov}_{G}(S, \alpha)$, mentioned in the introduction, in subsection 4.1. In subsection 4.2, we place a natural Borel measure on this space. Subsection 4.3 discusses why disconnected covers should be considered rare.

4.1. Spaces of covers. Let $S$ be a topological surface without boundary having infinite topological type (i.e., with infinitely generated fundamental group) which is realizable as a Riemann surface. By Radó's Theorem $S$ is second-countable [Rad25] [Hub06, §1.3]. Choose a basepoint $s_{0} \in S$. It follows from Richards' classification of surfaces [Ric63, Theorem 3] that the fundamental group $\pi_{1}\left(S, s_{0}\right)$ is isomorphic to the free group with countably many generators.

A covering of $S$ is a pair $(p, \tilde{S})$, where $\tilde{S}$ is a topological surface and $p: \tilde{S} \rightarrow S$ is a topological covering. Two covers $\left(p_{1}, \tilde{S}_{1}\right)$ and $\left(p_{2}, \tilde{S}_{2}\right)$ are isomorphic if there is a homeomorphism $h: \tilde{S}_{1} \rightarrow \tilde{S}_{2}$ so that $p_{1}=p_{2} \circ h$.

We recall idea of the monodromy action from covering space theory. Let $p: \tilde{S} \rightarrow S$ be a covering map. The monodromy action is the right action on the fiber over the basepoint, $p^{-1}\left(s_{0}\right)$, defined by

$$
p^{-1}\left(s_{0}\right) \times \pi_{1}\left(S, s_{0}\right) \rightarrow p^{-1}\left(s_{0}\right) ; \quad \tilde{s} \cdot \gamma:=\tilde{\beta}(1),
$$

where $\beta:[0,1] \rightarrow S$ is a loop in the class of $\gamma \in \pi_{1}\left(S, s_{0}\right)$, and $\tilde{\beta}:[0,1] \rightarrow \tilde{S}$ is a lift (i.e., $p \circ \tilde{\beta}=\beta$ ) so that $\tilde{\beta}(0)=\tilde{s}$. It should be noted that the definition of $\tilde{s} \cdot \gamma$ is independent of the choice of $\beta$. (Once $\beta$ is chosen, its lift $\tilde{\beta}$ is determined based on the condition that $\tilde{\beta}(0)=\tilde{s}$, and $\tilde{\beta}(1)$ depends only on $\gamma$.)

It is a basic observation from covering space theory that the monodromy action determines the cover up to isomorphism. This includes disconnected covers of the connected surface $S$. We will briefly show how to build a cover from an action. Concretely, given any right action of $\pi_{1}\left(S, s_{0}\right)$ on a discrete set $J$, we can build a cover of $S$ with this action as the monodromy action. To see this, fix such an action. For each $j \in J$, let $\operatorname{Stab}(j) \subset \pi_{1}\left(S, s_{0}\right)$ be the stabilizer of $j$. We can then build a cover $\tilde{S}_{j}$ as the quotient of the universal cover of $S$ by $\operatorname{Stab}(j)$. In the cover $\tilde{S}_{j}$, the lifts of the basepoint are then naturally identified with elements of the orbit $[j]$ of $j$ under the action of $\pi_{1}\left(S, s_{0}\right)$. It can be observed that if $k \in[j]$, then 
there is a cover isomorphism from $\tilde{S}_{j}$ to $\tilde{S}_{k}$ which respects the identification between $[j]$ and the lifts of the basepoint on each surface. It may be observed that by taking the disjoint union of such surfaces over all orbits under the action, we obtain a cover,

$$
\tilde{S}=\bigsqcup_{[j] \subset J} \tilde{S}_{j}
$$

of $S$ with the desired monodromy action. (Here we are picking a representative $j$ from each orbit [j].) Furthermore, actions on two discrete sets $J$ and $J^{\prime}$ determine isomorphic covers if and only if the actions are conjugate up to bijection, i.e., if there is a bijection $f: J \rightarrow J^{\prime}$ so that $f(j \cdot \gamma)=f(j) \cdot \gamma$ for all $\gamma \in \pi_{1}\left(S, s_{0}\right)$ and $j \in J$.

We will now specialize this discussion to finite covers of $S$. Suppose $p: \tilde{S} \rightarrow S$ is a covering map of degree $d$. Let $\Pi_{d}$ be the symmetric group acting by permutations of $\{1, \ldots, d\}$, and let

$$
\ell:\{1, \ldots, d\} \rightarrow p^{-1}\left(s_{0}\right)
$$

be a labeling (a bijection) to the fiber. The associated monodromy representation (which depends on the labeling) is the group homomorphism $M_{\ell}: \pi_{1}\left(S, s_{0}\right) \rightarrow \Pi_{d}$ defined so that

$$
M_{\ell}(\gamma)(i)=\ell^{-1}\left(\ell(i) \cdot \gamma^{-1}\right) \quad \text { for all } \gamma \in \pi_{1}\left(S, s_{0}\right) \text { and all } i \in\{1, \ldots, d\},
$$

where we have multiplied on the right by $\gamma^{-1}$ to make this map into a homomorphism (as opposed to an anti-homomorphism). Conversely, such a representation determines an action on $\{1, \ldots, d\}$ and so, from the above discussion, a choice of a monodromy representation $\pi_{1}\left(S, s_{0}\right) \rightarrow \Pi_{d}$ determines a $d$-fold cover of $S$. Given two such representations, the covers are isomorphic if and only if they differ by conjugation by an element of $\Pi_{d}$, which has the effect of changing the labeling function. Thus, the space of $d$-fold covers of $S$ up to isomorphism is canonically identified with

$$
\Pi_{d} \backslash \operatorname{Hom}\left(\pi_{1}\left(S, s_{0}\right), \Pi_{d}\right), \quad \text { where } \Pi_{d} \text { is acting by conjugation. }
$$

We endow $\operatorname{Hom}\left(\pi_{1}\left(S, s_{0}\right), \Pi_{d}\right)$ with the topology of pointwise convergence (or equivalently, the subspace topology coming from the inclusion of $\operatorname{Hom}\left(\pi_{1}\left(S, s_{0}\right), \Pi_{d}\right)$ into the product space $\left.\Pi_{d}^{\pi_{1}\left(S, s_{0}\right)}\right)$, and this space of covers gets the quotient topology.

As in the introduction if $G$ is a subgroup of $\Pi_{d}$ for an integer $d \geq 2$, we say that a cover $\tilde{S}$ has monodromy in $G$ if there is a representation $\pi_{1}\left(S, s_{0}\right) \rightarrow \bar{G}$ which determines a cover isomorphic to $\tilde{S}$. Note that this concept is independent of the basepoint. We define $\operatorname{Top} \operatorname{Cov}_{G}(S)$ to be the collection of all covers of $S$ with monodromy in $G$ up to isomorphism of covers. Such covers are thus determined by elements of $\operatorname{Hom}\left(\pi_{1}\left(S, s_{0}\right), G\right) \subset$ $\operatorname{Hom}\left(\pi_{1}\left(S, s_{0}\right), \Pi_{d}\right)$. So, the space $\operatorname{Top}_{\operatorname{Cov}_{G}}(S)$ is in bijective correspondence with

$$
\Pi_{d} \backslash \operatorname{Hom}\left(\pi_{1}\left(S, s_{0}\right), G\right) .
$$

We use the identification with this quotient space to topologize $\operatorname{TopCov}_{G}(S)$.

Proposition 8. The space $\operatorname{Top} \operatorname{Cov}_{G}(S)$ is homeomorphic to a Cantor set.

Proof. We use the characterization of Cantor sets as non-empty, perfect, compact, totally disconnected, and metrizable. We already know that $\operatorname{Hom}\left(\pi_{1}\left(S, s_{0}\right), G\right)$ is a Cantor set and thus satisfies these properties. Because $\operatorname{Top} \operatorname{Cov}_{G}(S)$ is a quotient of the Cantor set $\operatorname{Hom}\left(\pi_{1}\left(S, s_{0}\right), G\right)$, we see it is non-empty and compact. Because the equivalence classes have finite size, we see that because $\operatorname{Hom}\left(\pi_{1}\left(S, s_{0}\right), G\right)$ is perfect, so is $\operatorname{Top}_{G}(S)$. Also 
because of this, we can use a metric on $\operatorname{Hom}\left(\pi_{1}\left(S, s_{0}\right), G\right)$ and the use the Hausdorff metric restricted to equivalence classes to put a metric on $\operatorname{Top}_{\operatorname{Cov}}(S)$. To see the space is totally disconnected, suppose $h_{1}, h_{2} \in \operatorname{Hom}\left(\pi_{1}\left(S, s_{0}\right), G\right)$ have distinct images in $\operatorname{TopCov}_{G}(S)$. Then for any permutation $p \in \Pi_{d}$, there is an element $\gamma_{p} \in \pi_{1}\left(S, s_{0}\right)$ so that $h_{1}\left(\gamma_{p}\right) \neq p \cdot h_{2}\left(\gamma_{p}\right) \cdot p^{-1}$. For each permutation $q \in \Pi_{d}$, consider the following sets $U_{q} \subset \operatorname{Hom}\left(\pi_{1}\left(S, s_{0}\right), G\right)$ :

$$
U_{q}=\left\{h: h_{1}\left(\gamma_{p}\right)=q \cdot h\left(\gamma_{p}\right) \cdot q^{-1} \text { for all } p \in \Pi_{d}\right\} .
$$

The set $U_{q}$ is clopen because it is a finite union of cylinder sets. (To see this, we write each $\gamma_{p}$ as a product of generators. Then there are finitely many values $h$ can take on the generators used so that each $h\left(\gamma_{p}\right)$ equals $q^{-1} h_{1}\left(\gamma_{p}\right) q$, i.e., so that $h \in U_{q}$.) Also observe $h_{1} \in U_{e}$, where $e \in \Pi_{d}$ is the identity element, and $h_{2} \notin U_{q}$ for any $q$ from the remarks above. Thus the two complimentary sets

$$
U=\bigcup_{q \in \Pi_{d}} U_{q} \quad \text { and } \quad V=\bigcap_{q \in \Pi_{d}} \operatorname{Hom}\left(\pi_{1}\left(S, s_{0}\right), G\right) \backslash U_{q}
$$

are both clopen while $h_{1} \in U$ and $h_{2} \in V$. Finally, we observe they are invariant under conjugation since

$$
U=\bigcup_{q \in U_{q}} q^{-1} U_{e} q
$$

Thus $U$ and $V$ descend to clopen sets in $\Pi_{d} \backslash \operatorname{Hom}\left(\pi_{1}\left(S, s_{0}\right), G\right)$ which separate $\left[h_{1}\right]$ and $\left[h_{2}\right]$, proving that this quotient is totally disconnected.

Now suppose that we give the topological surface $S$ a translation structure, $(S, \alpha)$. As in the introduction, we use $\operatorname{Cov}_{G}(S, \alpha)$ to denote the space of covers of $(S, \alpha)$ with monodromy in $G$ up to translation equivalence. There is a natural map

$$
\operatorname{TopCov}_{G}(S) \rightarrow \operatorname{Cov}_{G}(S, \alpha)
$$

which sends a topological covering $p: \tilde{S} \rightarrow S$ to $(\tilde{S}, \tilde{\alpha})$ where $\tilde{\alpha}$ is obtained by pulling back $\alpha$ under $p$. This map is clearly surjective, and we endow $\operatorname{Cov}_{G}(S, \alpha)$ with the finest topology so that this natural map is continuous. In other words, translation equivalence induces an equivalence relation on $\operatorname{Top}_{\operatorname{Cov}}(S)$, and $\operatorname{Cov}_{G}(S, \alpha)$ as a topological space is naturally identified with the resulting quotient.

Remark 9. Note that our definition of translation automorphism does no consider basepoints. So, it is possible for two covers $\left(\tilde{S}_{1}, \tilde{\alpha}_{1}\right)$ and $\left(\tilde{S}_{2}, \tilde{\alpha}_{2}\right)$ of $(S, \alpha)$ to be translation equivalent without arising from an isomorphism of covering maps. However such pairs of can be ruled out if the surface $(S, \alpha)$ is geometrically primitive in the sense the deck group of the universal cover of $S$ is the same as the translation automorphism group of the universal cover (when endowed with the pullback translation structure).

Observe that a homeomorphism $\phi: S \rightarrow S^{\prime}$ between topological surfaces induces a homeomorphism between their spaces of covers with monodromy in $G$ up to cover isomorphism. This is easily seen through the fundamental group. In order to consider their fundamental groups, we choose basepoints $s_{0}$ and $s_{0}^{\prime}$. To identify the fundamental groups, we make a choice of a curve $\beta:[0,1] \rightarrow S^{\prime}$ so that $\beta(0)=\phi\left(s_{0}\right)$ and $\beta(1)=s_{0}^{\prime}$. The choice of $\beta$ gives rise to a group homomorphism

$$
\phi_{\beta}: \pi_{1}\left(S, s_{0}\right) \rightarrow \pi_{1}\left(S^{\prime}, s_{0}^{\prime}\right) ;[\gamma] \mapsto\left[\beta^{-1} \bullet(\phi \circ \gamma) \bullet \beta\right],
$$


where $\bullet$ denotes path concatenation (with the path on the left being traversed first). This action allows $\phi$ to act on the spaces of topological covers with monodromy in $G$. By identifying these spaces of covers as in equation 6, we see that this action is given by

$$
\operatorname{TopCov}_{G}(S) \rightarrow \operatorname{TopCov}_{G}\left(S^{\prime}\right) ; \quad[h] \mapsto\left[h \circ \phi_{\beta}^{-1}\right]
$$

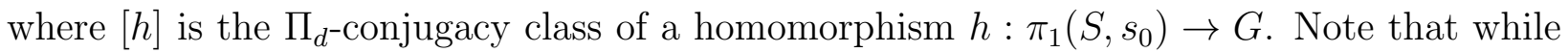
the action of $\phi$ on the fundamental group depended on the choice of the curve $\beta$, the action on this space of covers does not, since the map $h \mapsto h \circ \phi_{\beta}^{-1}$ only changes by post-conjugation by a permutation when $\beta$ is changed.

A homeomorphism $(S, \alpha) \rightarrow\left(S^{\prime}, \alpha^{\prime}\right)$ between translation surfaces does not necessarily induce a homeomorphism from $\operatorname{Cov}_{G}(S, \alpha)$ to $\operatorname{Cov}_{G}\left(S^{\prime}, \alpha^{\prime}\right)$. (This fails for instance, if $(S, \alpha)$ admits translation automorphisms and $\left(S^{\prime}, \alpha^{\prime}\right)$ does not.) However, an affine homeomorphism does induce such a homeomorphism. As above, this homeomorphism is induced by the map $h \mapsto h \circ \phi_{\beta}^{-1}$. We summarize this observation below.

Proposition 10. Let $\phi:(S, \alpha) \rightarrow\left(S^{\prime}, \alpha^{\prime}\right)$ be an affine homeomorphism with derivative $A \in G L(2, \mathbb{R})$, and let $\phi_{\beta}: \pi_{1}\left(S, s_{0}\right) \rightarrow \pi_{1}\left(S, s_{0}^{\prime}\right)$ be the group homomorphism as defined above for some choice of curve $\beta$. If $(\tilde{S}, \tilde{\alpha})$ is a cover of $(S, \alpha)$ with monodromy homomorphism $h$ : $\pi_{1}\left(S, s_{0}\right) \rightarrow G$, then $A(\tilde{S}, \tilde{\alpha})$ is translation equivalent to the cover of $\left(S^{\prime}, \alpha^{\prime}\right)$ with monodromy homomorphism $h \circ \phi_{\beta}^{-1}$. Thus, map $h \mapsto h \circ \phi_{\beta}^{-1}$ induces a homeomorphism $A_{*}: \operatorname{Cov}_{G}(S, \alpha) \rightarrow$ $\operatorname{Cov}_{G}\left(S^{\prime}, \alpha^{\prime}\right)$, which depends only on the derivative $A$ of the affine homeomorphism $\phi$.

As a consequence of this proposition, we observe that the Veech group of $(S, \alpha)$ acts on $\operatorname{Cov}_{G}(S, \alpha)$ :

Corollary 11 (The Veech group acts on covers). Let $A \in S L_{ \pm}(2, \mathbb{R})$ be an element of the Veech group of $(S, \alpha)$, and let $(\tilde{S}, \tilde{\alpha}) \in \operatorname{Cov}_{G}(S, \alpha)$. Then $A(\tilde{S}, \tilde{\alpha})$ is also in $\operatorname{Cov}_{G}(S, \alpha)$.

4.2. Measures on spaces of covers. In this subsection, we will construct some natural measures on our spaces of covers. We begin by describing an abstract construction. Later in the subsection, we will specialize the discussion to our setting of translation surfaces.

Let $\Gamma^{+}$be the non-abelian free group with a countable generating set $\left\{\gamma_{i}: i \in \mathbb{N}\right\}$, and let $G \subset \Pi_{d}$ as above. We endow the space $\operatorname{Hom}\left(\Gamma^{+}, G\right)$ with its natural product topology, which makes the $\operatorname{Hom}\left(\Gamma^{+}, G\right)$ homeomorphic to a Cantor set. This is the coarsest topology so that for each $\eta \in \Gamma^{+}$and each $\sigma \in G$, the set of the form

$$
\left\{h: \operatorname{Hom}\left(\Gamma^{+}, G\right): h(\eta)=\sigma\right\} .
$$

is open. Two ordered $k$-tuples of distinct elements $\left(e_{1}, \ldots, e_{k}\right) \in \mathbb{N}^{k}$ and $\left(\sigma_{1}, \ldots, \sigma_{k}\right) \in G^{k}$, determine a cylinder set in $\operatorname{Hom}\left(\Gamma^{+}, G\right)$,

$$
\mathcal{C}\left(e_{1}, \ldots, e_{k} ; \sigma_{1}, \ldots, \sigma_{k}\right)=\left\{h: \operatorname{Hom}\left(\Gamma^{+}, G\right): h\left(\gamma_{e_{i}}\right)=\sigma_{i} \text { for } i=1, \ldots, k\right\} .
$$

Each cylinder set is both closed and open in the product topology, and the collection of cylinder sets generate the topology.

To characterize a Borel measure on $\operatorname{Hom}\left(\Gamma^{+}, G\right)$, it suffices to describe the measures of the cylinder sets.

Definition 12. The product measure $\mu$ on $\operatorname{Hom}\left(\Gamma^{+}, G\right)$ is defined so that for every cylinder set we have

$$
\mu\left(\mathcal{C}\left(e_{1}, \ldots, e_{k} ; \sigma_{1}, \ldots, \sigma_{k}\right)\right)=\frac{1}{|G|^{k}}
$$


This is the product measure induced on $\operatorname{Hom}\left(\Gamma^{+}, G\right)$ by the counting measure on $G$.

We remark that this measure $\mu$ is interesting even in the case when $\Gamma^{+}$is a finitely generated free group, and related questions remain open [Pud13].

Automorphisms of $\Gamma^{+}$act on $\operatorname{Hom}\left(\Gamma^{+}, G\right)$. Concretely, if $\phi: \Gamma^{+} \rightarrow \Gamma^{+}$is an automorphism, then we can define

$$
\phi_{*}: \operatorname{Hom}\left(\Gamma^{+}, G\right) \rightarrow \operatorname{Hom}\left(\Gamma^{+}, G\right) ; \quad h \mapsto h \circ \phi^{-1} .
$$

Lemma 13. The action of any automorphism of $\Gamma^{+}$preserves the product measure $\mu$ on $\operatorname{Hom}\left(\Gamma^{+}, G\right)$. In particular, the measure $\mu$ is independent of our choice of generating set.

Remark 14 (Proof in abelian case). In the case when $G$ is abelian, $\operatorname{Hom}\left(\Gamma^{+}, G\right)$ can be identified with the topological group $G^{\mathbb{N}}$, and $\mu$ is Haar measure. In this case, the proposition follows from the naturality of Haar measure.

Proof. Let $\phi: \Gamma^{+} \rightarrow \Gamma^{+}$be an automorphism, and let $\phi_{*}$ be its action on $\operatorname{Hom}\left(\Gamma^{+}, G\right)$ :

$$
\phi_{*}: \operatorname{Hom}\left(\Gamma^{+}, G\right) \rightarrow \operatorname{Hom}\left(\Gamma^{+}, G\right) ; \quad h \mapsto h \circ \phi^{-1} .
$$

We will prove that $\phi_{*}$ preserves the product measure $\mu$ on $\operatorname{Hom}\left(\Gamma^{+}, G\right)$. It suffices to prove that the measures of cylinder sets are preserved. Let $\mathcal{C}=\mathcal{C}\left(e_{1}, \ldots, e_{k} ; \sigma_{1}, \ldots, \sigma_{k}\right)$ be a cylinder set. We will prove that $\mu \circ \phi_{*}(\mathcal{C})=1 /|G|^{k}$.

Let $X=\left\langle\gamma_{e_{1}}, \ldots, \gamma_{e_{k}}\right\rangle \subset \Gamma^{+}$. Since $\Gamma^{+}=\left\langle\gamma_{e}: e \in \mathbb{N}\right\rangle$, there is a finite set $\left\{e_{1}^{\prime}, \ldots, e_{m}^{\prime}\right\} \subset$ $\mathbb{N}$ so that

$$
\phi^{-1}(X) \subset\left\langle\gamma_{e_{1}^{\prime}}, \ldots, \gamma_{e_{m}^{\prime}}\right\rangle .
$$

We'll call the subgroup on the right hand side of the equation $Y$. By viewing $\mu$ as the product of counting measures, we see

$$
\mu \circ \phi^{*}(\mathcal{C})=\frac{\#\left\{h \in \operatorname{Hom}(Y, G): h \circ \phi^{-1}\left(\gamma_{e_{i}}\right)=\sigma_{i} \quad \text { for } 1 \leq i \leq k\right\}}{|G|^{m}} .
$$

So it suffices to show that the number of homomorphisms in the numerator is $|G|^{m-k}$.

As above, we can find a finite set $\left\{e_{1}^{\prime \prime}, \ldots, e_{n}^{\prime \prime}\right\} \subset \mathbb{N}$ so that

$$
\phi(Y) \subset\left\langle\gamma_{e_{1}^{\prime \prime}}, \ldots, \gamma_{e_{n}^{\prime \prime}}\right\rangle .
$$

Call the set on the right hand side $Z$. Note that $X \subset Z$.

We recall some basic definitions from the theory of free groups. A basis of a free group $F$ is a set $x_{1}, \ldots, x_{k}$ so that $F=\left\langle x_{1}\right\rangle * \ldots *\left\langle x_{k}\right\rangle$. A subgroup $H$ of a free group $F$ is a free factor if every (equivalently, some) basis of $H$ can be extended to a basis of $F$.

Consider $X, Y$, and $Z$ as above. Observe that $X$ is a free factor in $Z$. So, $X$ is a free factor in $\phi(Y)$ Pud13, Claim 2.5]. That is, we can extend $\left\{\gamma_{e_{1}}, \ldots, \gamma_{e_{k}}\right\}$ to a free generating set of $\phi(Y)$. Using $\phi^{-1}$, we can pull this back to a generating set of $Y$. So, we have $k \leq n$, and there is a free generating set of $Y$ given by $\beta_{1}, \ldots, \beta_{m}$ so that

$$
\beta_{i}=\phi^{-1}\left(\gamma_{e_{i}}\right) \text { for } 1 \leq i \leq k .
$$

Since this set generates $Y$, we see that $\operatorname{Hom}(Y, G)$ is in bijective correspondence with the possible images of $\left\{\beta_{1}, \ldots, \beta_{m}\right\}$. The last $m-k$ elements in this basis are irrelevant to the values of $\phi^{-1}\left(\gamma_{e_{i}}\right)$, so we see that there are exactly $|G|^{m-k}$ possible values which give homomorphisms in the numerator of equation 12 . 
Recall that whenever $S$ is a topological surface of infinite topological type, then its fundamental group $\pi_{1}\left(S, s_{0}\right)$ is isomorphic to a countably generated free group. It is a simple observation that conjugation by a permutation preserves the measure $\mu_{G}$ constructed above. By identifying $\operatorname{Hom}\left(\Gamma^{+}, G\right)$ with $\operatorname{Hom}\left(\pi_{1}\left(S, s_{0}\right), G\right)$ and $\operatorname{TopCov}_{G}(S)$ with $\Pi_{d} \backslash \operatorname{Hom}\left(\pi_{1}\left(S, s_{0}\right), G\right)$, we obtain a measure $\nu_{G}$ on $\operatorname{Top}_{\operatorname{Cov}}(S)$, which we call the product measure on $\operatorname{Top} \operatorname{Cov}_{G}(S)$.

Corollary 15. Let $\phi: S \rightarrow S^{\prime}$ be a homeomorphism between two topological surfaces of infinite topological type. Let $G \subset \Pi_{d}$ for $d \geq 2$, and let $\nu_{G}$ and $\nu_{G}^{\prime}$ be the product measures on $\operatorname{Top}_{\operatorname{Cov}_{G}}(S)$ and $\operatorname{Top} \operatorname{Cov}_{G}\left(S^{\prime}\right)$, respectively. Then, $\nu_{G}^{\prime}$ is the pushforward of the measure $\nu_{G}$ under the map $\operatorname{Top}_{\operatorname{Cov}_{G}}(S) \rightarrow \operatorname{Top} \operatorname{Cov}_{G}\left(S^{\prime}\right)$ induced by $\phi$ as in equation 9 .

Proof. We can identify each space of covers with $\operatorname{Hom}\left(\Gamma^{+}, G\right)$ using isomorphisms to the fundamental groups. As noted in equation 9, the action of a homeomorphism on the space of covers is induced by a group isomorphism between the fundamental groups and via our identifications, an automorphism of $\Gamma^{+}$. Lemma 13 tells us that the measure $\mu_{G}$ is invariant under such automorphisms. Also the automorphism commutes with the (partially defined) $\Pi_{d^{-}}$action. Thus, our measures $\nu_{G}$ and $\nu_{G}^{\prime}$ are the same in view of the identification of each space of covers with $\Pi_{d} \backslash \operatorname{Hom}\left(\Gamma^{+}, G\right)$.

Now let $(S, \alpha)$ be a translation surface of infinite topological type. $\operatorname{Since}_{\operatorname{Cov}_{G}}(S, \alpha)$ is a quotient of $\operatorname{Top} \operatorname{Cov}_{G}(S)$, we obtain an measure $m_{G}$ on $\operatorname{Cov}_{G}(S, \alpha)$ as the pushforward of $\nu_{G}$. We call $m_{G}$ the product measure on $\operatorname{Cov}_{G}(S, \alpha)$.

Recall that Proposition 10 says that when $A \in S L(2, \mathbb{R})$ and $A(S, \alpha)$ is translation equivalent to $\left(S^{\prime}, \alpha^{\prime}\right)$, there is an induced homeomorphism $A_{*}$ from $\operatorname{Cov}_{G}(S, \alpha)$ to $\operatorname{Cov}_{G}\left(S^{\prime}, \alpha^{\prime}\right)$. This homeomorphism respects the product measures on these spaces:

Corollary 16 (Affine naturality of measures). Let $(S, \alpha)$ be a translation surface of infinite topological type. Let $A \in S L_{ \pm}(2, \mathbb{R})$ and let $\left(S^{\prime}, \alpha^{\prime}\right)=A(S, \alpha)$. Then $m_{G}^{\prime}=m_{G} \circ A_{*}^{-1}$ where $m_{G}$ and $m_{G}^{\prime}$ are the product measures on $\operatorname{Cov}_{G}(S, \alpha)$ and $\operatorname{Cov}_{G}\left(S^{\prime}, \alpha^{\prime}\right)$.

Proof. Because $A(S, \alpha)=\left(S^{\prime}, \alpha^{\prime}\right)$, there must be an affine homeomorphism $\phi:(S, \alpha) \rightarrow$ $\left(S^{\prime}, \alpha^{\prime}\right)$ with derivative $A$. The homeomorphism $A_{*}: \operatorname{Cov}_{G}(S, \alpha) \rightarrow \operatorname{Cov}_{G}\left(S^{\prime}, \alpha^{\prime}\right)$ lifts to a homeomorphism $\Phi: \operatorname{Top}_{\operatorname{Cov}_{G}}(S, \alpha) \rightarrow \operatorname{Top} \operatorname{Cov}_{G}\left(S^{\prime}, \alpha^{\prime}\right)$ by Proposition 10. Since $\Phi_{*}\left(\nu_{G}\right)=$ $\nu_{G}^{\prime}$ by Corollary 15 and $m_{G}$ and $m_{G}^{\prime}$ are obtained as images of $\nu_{G}$ and $\nu_{G}^{\prime}$, we see $m_{G}^{\prime}=$ $m_{G} \circ A_{*}^{-1}$.

4.3. Disconnected covers. Let $G \subset \Pi_{d}$ and let $h \in \operatorname{Hom}\left(\pi_{1}\left(S, s_{0}\right), G\right)$. By interpreting $h$ as the monodromy action of the fundamental group of $S$ on the fibers of the basepoint, we obtain a cover $\tilde{S}$ of $S$ as in $\S 4.1$. This cover is explicitly described by equation (5), and we can see the following:

Proposition 17. The cover associated to $h \in \operatorname{Hom}\left(\pi_{1}\left(S, s_{0}\right), G\right)$ is connected if and only if the image $h\left(\pi_{1}\left(S, s_{0}\right)\right)$ acts transitively on $\{1,2, \ldots, d\}$.

In particular, in order to have connected covers of $(S, \alpha)$ with monodromy in $G$, the subgroup $G \subset \Pi_{d}$ must act transitively on $\{1,2, \ldots, d\}$. The goal of this subsection is to formulate the following precise version of the statement that the collection of all disconnected covers is small.

Proposition 18. Let $G \subset \Pi_{d}$ be a subgroup which acts transitively on $\{1,2, \ldots, d\}$. Let $S$ be a topological surface of infinite topological type. Then, $\nu_{G}$-almost every cover in $\operatorname{TopCov}_{G}(S)$ is connected. 
Proof. Let $\mathcal{H}$ denote the collection of all subgroups of $H \subset G$ so that $H$ does not act transitively on $\{1, \ldots, d\}$. Note that $\mathcal{H}$ is a finite set. Consider the set $\mathcal{D} \subset \operatorname{Cov}_{G}(S, \alpha)$ of disconnected covers with monodromy in $G$. Recall that $\operatorname{Cov}_{G}(S, \alpha)$ is a quotient of $\operatorname{Hom}\left(\pi_{1}\left(S, s_{0}\right), G\right)$. Let $\tilde{\mathcal{D}} \subset \operatorname{Hom}\left(\pi_{1}\left(S, s_{0}\right), G\right)$ be the lift of $\mathcal{D}$. By definition of $\nu_{G}$, we have $\nu_{G}(\mathcal{D})=\mu_{G}(\tilde{\mathcal{D}})$. Proposition 17 tells us that

$$
\tilde{\mathcal{D}}=\bigcup_{H \in \mathcal{H}} \operatorname{Hom}\left(\pi_{1}\left(S, s_{0}\right), H\right)
$$

Let $\tilde{\mathcal{D}}_{H}=\operatorname{Hom}\left(\pi_{1}\left(S, s_{0}\right), H\right)$. By subadditivity of measures, it suffices to prove that $\mu_{G}\left(\tilde{\mathcal{D}}_{H}\right)=0$ for all $H \in \mathcal{H}$.

Fix $H \in \mathcal{H}$. Observe that $H$ is a proper subgroup of $G$, since $H$ does not act transitively while $G$ does. Fix some $\epsilon>0$. We will show that $\mu_{G}\left(\tilde{\mathcal{D}}_{H}\right)<\epsilon$. Since $H$ is a proper subset of $G$, we can find a $k$ so that $\left(\frac{|H|}{|G|}\right)^{k}<\epsilon$. Observe that $\tilde{\mathcal{D}}_{H}$ is contained in the union of cylinder sets

$$
\bigcup_{\left(h_{1}, \ldots, h_{k}\right) \in H^{k}} \mathcal{C}\left(1, \ldots, k ; h_{1}, \ldots, h_{k}\right),
$$

where we are using notation from equation 10 . Observe that by monotonicity and by Definition 12 of $\mu_{G}$, we have

$$
\mu_{G}\left(\tilde{\mathcal{D}}_{H}\right) \leq \sum_{\left(h_{1}, \ldots, h_{k}\right) \in H^{k}} \mu_{G}\left(\mathcal{C}\left(1, \ldots, k ; h_{1}, \ldots, h_{k}\right)\right)=\frac{|H|^{k}}{|G|^{k}}<\epsilon .
$$

This proves that $\mu_{G}\left(\tilde{\mathcal{D}}_{H}\right)=0$, and thus $\nu_{G}(\mathcal{D})=0$ by the remarks in the previous paragraph.

\section{ERGODICITY}

Let $(S, \alpha)$ be a flat surface and let $G$ be a subgroup of the permutation group $\Pi_{d}$ for some integer $d \geq 2$. The group $S L_{ \pm}(2, \mathbb{R})$ acts on the the space of affine deformations of covers with monodromy in $G, \tilde{\mathcal{O}}_{G}(S, \alpha)$, and the action of the diagonal subgroup, $g^{t}$, is the cover cocycle. (See (2).)

In this section, we prove Theorem 1, which pertains to a connected cover $(\tilde{S}, \tilde{\alpha}) \in$ $\operatorname{Cov}_{G}(S, \alpha)$ : If the Teichmüller trajectory (covers cocycle orbit) $g^{t}(\tilde{S}, \tilde{\alpha})$ has an accumulation point in $\tilde{\mathcal{O}}_{G}(S, \alpha)$ representing a connected surface, then the translation flow on $(\tilde{S}, \tilde{\alpha})$ is defined for all time almost everywhere and is ergodic.

We will see that Theorem 1 is a consequence of the following result, which gives a criterion for ergodicity in terms of the geometries realized under the Teichmüller deformation. Before stating the Theorem we establish some notation. Let $\Sigma \subset \bar{S}$ be the subset of the metric completion of $S$ defined as the union of the zeros of $\alpha$ and the points in $\bar{S} \backslash S$. For $t \in \mathbb{R}$ we define dist $_{t}$ to be the metric on $S$ obtained by pulling back the flat metric on $g^{t}(S, \alpha)$ under the affine homeomorphism $(S, \alpha) \rightarrow g^{t}(S, \alpha)$ with derivative $g_{t}$.

Theorem 19 ([Tre14, Theorem 3]). Let $(S, \alpha)$ be a flat surface of finite area. Suppose that for any $\eta>0$ there exist a function $t \mapsto \varepsilon(t)>0$, a one-parameter family of subsets

$$
S_{t}=\bigsqcup_{i=1}^{C_{t}} S_{t}^{i}
$$


of $S$ made up of $C_{t}<\infty$ path-connected components, each homeomorphic to a closed orientable surface with boundary, and functions $t \mapsto \mathcal{D}_{t}^{i}>0$, for $1 \leq i \leq C_{t}$, such that for

$$
\Gamma_{t}^{i, j}=\left\{\text { paths connecting } \partial S_{t}^{i} \text { to } \partial S_{t}^{j}\right\}
$$

and

$$
\delta_{t}=\min _{i \neq j} \sup _{\gamma \in \Gamma_{t}^{i, j}} \operatorname{dist}_{t}(\gamma, \Sigma)
$$

the following hold:

(1) Area $\left(S \backslash S_{t}\right)<\eta \operatorname{Area}(S)$ for all $t>0$,

(2) $\operatorname{dist}_{t}\left(\partial S_{t}, \Sigma\right)>\varepsilon(t)$ for all $t>0$,

(3) the diameter of each $S_{t}^{i}$, measured using dist $t_{t}$, is bounded above by $\mathcal{D}_{t}^{i}$ and

$$
\int_{0}^{\infty}\left(\varepsilon(t)^{-2} \sum_{i=1}^{C_{t}} \mathcal{D}_{t}^{i}+\frac{C_{t}-1}{\delta_{t}}\right)^{-2} d t=+\infty .
$$

Then the translation flow is defined for all time almost everywhere and is ergodic.

The theorem above is a geometric criterion for ergodicity. The spirit of the theorem is that if, as ones deforms a flat surface $(S, \alpha)$ using the Teichmüller deformation $g^{t}$, the geometry of the surface does not deteriorate too quickly (as measured by the diameter of big components, among other things), the translation flow is ergodic.

In [Tre14], this theorem was proved with the additional hypothesis that the set of points whose trajectories leave every compact subset of $S$ has zero measure. This is equivalent to the statement that the translation flow is defined for all time almost everywhere (which we state as a conclusion above). To get the version above we need to prove that the hypothesis is unnecessary:

Proof that the translation flow is defined for all time almost everywhere. We will show that the geometric conditions listed above force the translation flow to be defined for all time almost everywhere. Suppose $(S, \alpha)$ is a finite area translation surface and satisfies the list of geometric conditions given in the Theorem. Assume to the contrary that the translation flow $F^{s}$ is not defined for all time almost everywhere. Then there is a time $s_{0}$ and a measurable subset $X \subset S$ of Lebesgue measure $m>0$ so that $F^{s_{0}}(x)$ is undefined for all $x \in X$. There is a geometric consequence to lying in $X$ : For any $x \in X$ there is a $s_{x} \in \mathbb{R}$ with $0<s_{x} \leq s_{0}$ so that $F^{s}(x)$ is defined for $s \in\left[0, s_{x}\right)$ and

$$
\lim _{s \rightarrow s_{x^{-}}} F^{s}(x) \in \Sigma .
$$

In particular, the distance from $x$ to $\Sigma$ measured with dist $t_{t}$ is no larger than $s_{x} e^{-t} \leq s_{0} e^{-t}$.

Select an $\eta$ so that $m>\eta \operatorname{Area}(S)$. Then any subsurface $S_{t}$ satisfying condition (1) of the theorem must satisfy $S_{t} \cap X \neq \emptyset$. As a consequence of condition (2) and remarks above, we must have $\varepsilon(t)<s_{0} e^{-t}$.

We will draw a contradiction to the integral (14) being infinite. To do this it suffices to get some control of the sum of the diameters. We partition the set $\{t \in \mathbb{R}: t \geq 0\}$ into two pieces $\mathcal{S}$ and $\mathcal{L}$ (for "small" and "large"). We declare $t$ to lie in $\mathcal{S}$ if all components $S_{t}^{i}$ have diameter less than or equal to $\varepsilon(t)$, and declare $t$ to lie in $\mathcal{L}$ otherwise. We will control the sum $\sum_{i} \mathcal{D}_{t}^{i}$ by separate arguments on the two sets. 
Suppose $t \in \mathcal{S}$. Then all components of $S_{t}$ have diameter less than $\varepsilon(t)$. Let $x_{i} \in S_{t}^{i}$ be a point in one of the components. Since $\operatorname{dist}_{t}\left(x_{i}, \Sigma\right)>\varepsilon(t)$ by hypothesis, we can embed a Euclidean $B_{i}$ ball of radius $\mathcal{D}_{t}^{i}<\varepsilon(t)$ about $x_{i}$ within $S$ using the distance dist $t_{t}$ Then we have $S_{t}^{i} \subset B_{i}$, so

$$
\operatorname{Area}\left(S_{t}^{i}\right) \leq \operatorname{Area}\left(B_{i}\right)=\pi\left(\mathcal{D}_{t}^{i}\right)^{2}
$$

Observe this holds for all $i$ and so it follows that the sum $\sum_{i} \mathcal{D}_{t}^{i}$ is bounded from below by a constant:

$$
\left(\sum_{i} \mathcal{D}_{t}^{i}\right)^{2} \geq \sum_{i}\left(\mathcal{D}_{t}^{i}\right)^{2} \geq \frac{4}{\pi} \sum_{i} \operatorname{Area}\left(S_{t}^{i}\right)=\frac{1}{\pi} \operatorname{Area}\left(S_{t}\right)>\frac{1}{\pi}(1-\eta) \operatorname{Area}(S) .
$$

Now suppose $t \in \mathcal{L}$. Then $S_{t}$ has a component of diameter at least $\varepsilon(t)$. In this case we can use the very naïve bound

$$
\sum_{i} \mathcal{D}_{t}^{i} \geq \varepsilon(t)
$$

Combining (15) and (16), we see that $\sum_{i} \mathcal{D}_{t}^{i} \geq D(t)$ where $D(t)$ is defined by

$$
D(t)=\min \left\{\varepsilon(t), \sqrt{\frac{1}{\pi}(1-\eta) \operatorname{Area}(S)}\right\} .
$$

The quantity on the right is a constant while $\varepsilon(t)$ tends to zero since $\varepsilon(t)<s_{0} e^{-t}$. Thus $D(t)=\varepsilon(t)$ for $t>t_{*}$ for some $t_{*} \geq 0$. For $t>t_{*}, \sum_{i} \mathcal{D}_{t}^{i} \geq \varepsilon(t)$ and so the quantity being integrated in (14) satisfies

$$
\left(\varepsilon(t)^{-2} \sum_{i=1}^{C_{t}} \mathcal{D}_{t}^{i}+\frac{C_{t}-1}{\delta_{t}}\right)^{-2} \leq\left(\varepsilon(t)^{-2} \varepsilon(t)\right)^{-2}=\varepsilon(t)^{2} .
$$

Recalling $\varepsilon(t)<s_{0} e^{-t}$, we see the total integral (14) is bounded by

$$
\int_{0}^{t_{*}}\left(\varepsilon(t)^{-2} \sum_{i=1}^{C_{t}} \mathcal{D}_{t}^{i}+\frac{C_{t}-1}{\delta_{t}}\right)^{-2} d t+s_{0}^{2} \int_{t_{*}}^{\infty} e^{-2 t} d t
$$

which is finite. This is our contradiction.

A key consequence for us is the following which was mentioned in the introduction:

Corollary 20 ([Tre14, Theorem 2]). Suppose $(S, \alpha)$ is a finite area translation surface of infinite topological type. Let $a \in S L_{ \pm}(2, \mathbb{R})$. Then, if the trajectory $g^{t} a(S, \alpha)$ is non-divergent in $\mathcal{O}(S, \alpha)$, then the translation flow on $a(S, \alpha)$ is defined for all time almost everywhere and is ergodic.

It is worth noting that in [Tre14] this was proved independently from Theorem 19. We will prove this here using Theorem 19 because we will use some of the same ideas in the proof of Theorem 1 .

Proof. By replacing $(S, \alpha)$ with $a(S, \alpha)$, we can assume that $a$ is the identity. We will use square brackets to denote the translation equivalence class of a translation surface in $\mathcal{O}(S, \alpha)$. Non-divergence guarantees that there is sequence of times $t_{k}$ tending to $+\infty$ so that the translation equivalence class $\left[g^{t_{k}}(S, \alpha)\right]$ converges in $\mathcal{O}(S, \alpha)$ to some limit $[b(S, \alpha)]$. Since $\mathbb{N} \cup\{+\infty\}$ with its usual topology is compact and the map $S L(2, \mathbb{R}) \rightarrow \mathcal{O}(S, \alpha)$ given by $a \mapsto[a(S, \alpha)]$ is continuous, $K=\left\{\left[g^{t_{k}}(S, \alpha)\right]: k \in \mathbb{N}\right\} \cup\{[b(S, \alpha)]\}$ is compact in 
$\mathcal{O}(S, \alpha)$. Fix any $\eta>0$. Select a compact connected subsurface with boundary $L \subset S$ whose area is greater than $1-\eta$ times the area of $S$, and so that $L$ does not include any zeros of $\alpha$. Fix any $\epsilon>0$. For $t \in\left[t_{k}-\epsilon, t_{k}+\epsilon\right]$ select the subsurface $S_{t}=g^{-t_{k}} b_{k}(L)$ (or any such subsurface if $t$ belongs to multiple such intervals). Then since $g^{t}(S, \alpha)$ is translation equivalent to $g^{t-t_{k}} b_{k}(S, \alpha)$, there is an isometry from $S$ with the metric dist $t_{t}$ to the translation surface $g^{t-t_{k}} b_{k}(S, \alpha)$ which carries $S_{t}$ to the image of $L$ under $g^{t-t_{k}} b_{k}$. So for all $t$ in any $\left[t_{k}-\epsilon, t_{k}+\epsilon\right]$, the subsurface we have selected is isometric to the image of $m(L)$ under an $m \in g^{[-\epsilon, \epsilon]} K$ viewed as a subsurface of $m(S, \alpha)$. Observe that the quantities in Theorem 19 vary continuously in $m$ as we deform the metric in this way. Thus, the quantity being integrated over times $t \in \bigcup_{k}\left[t_{k}-\epsilon, t_{k}+\epsilon\right]$ is bounded uniformly from below by a uniform positive constant. Since $t_{k}$ is an infinite sequence tending to $+\infty$, the integral is infinite.

Proof of Theorem 1. Let $(S, \alpha)$ be a finite area translation surface with infinite topological type, and let $(\tilde{S}, \tilde{\alpha})$ be a cover with monodromy in $G \subset \Pi_{d}$. We assume that $\left[g^{t}(\tilde{S}, \tilde{\alpha})\right]$ has an $\omega$-limit point in $\tilde{\mathcal{O}}_{G}(S, \alpha)$ which is the translation equivalence class of a connected surface. Let $t_{k}$ be a sequence of times tending to $+\infty$ for which $\left[g^{t_{k}}(\tilde{S}, \tilde{\alpha})\right]$ approaches this $\omega$-limit. Then by definition of the topology on $\tilde{\mathcal{O}}_{G}(S, \alpha)$, there is a sequence

$$
\left(b_{k},\left[\left(\tilde{S}_{k}, \tilde{\alpha}_{k}\right)\right]\right) \in S L(2, \mathbb{R}) \times \operatorname{Cov}_{G}(S, \alpha)
$$

so that for all $k$ the surface $b_{k}\left(\tilde{S}_{k}, \tilde{\alpha}_{k}\right)$ is translation equivalent to $g^{t_{k}}(\tilde{S}, \tilde{\alpha})$, the sequence $b_{k}$ converges to some $b \in S L(2, \mathbb{R})$ and the sequence $\left[\left(\tilde{S}_{k}, \tilde{\alpha}_{k}\right)\right]$ converges to some connected $\left[\left(\tilde{S}_{\infty}, \tilde{\alpha}_{\infty}\right)\right] \in \operatorname{Cov}_{G}(S, \alpha)$.

We will briefly discuss the convergence of $\left[\left(\tilde{S}_{k}, \tilde{\alpha}_{k}\right)\right]$ to the connected cover $\left[\left(\tilde{S}_{\infty}, \tilde{\alpha}_{\infty}\right)\right]$ within $\operatorname{Cov}_{G}(S, \alpha)$. Fix a basepoint $s_{0} \in S$ which is not a zero. Choose a representative $\operatorname{cover}\left(\tilde{S}_{\infty}, \tilde{\alpha}_{\infty}\right)$ from $\left[\left(\tilde{S}_{\infty}, \tilde{\alpha}_{\infty}\right)\right]$. Since $\left[\left(\tilde{S}_{\infty}, \tilde{\alpha}_{\infty}\right)\right] \in \operatorname{Cov}_{G}(S, \alpha)$, we can denote the lifts of $s_{0}$ by $s_{\infty}^{1}, \ldots, s_{\infty}^{d} \in \tilde{S}_{\infty}$ obtaining a monodromy homomorphism $h_{\infty}: \pi_{1}\left(S, s_{0}\right) \rightarrow G \subset \Pi_{d}$. From the definition of the topology $\operatorname{Cov}_{G}(S, \alpha)$, for all $k$ we can select covers $\left(\tilde{S}_{k}, \tilde{\alpha}_{k}\right)$ from the equivalence classes $\left[\left(\tilde{S}_{k}, \tilde{\alpha}_{k}\right)\right]$ and denote the lifts of the basepoint $s_{0}$ by $s_{k}^{1}, \ldots, s_{k}^{d} \in \tilde{S}_{k}$ in such a way so that the corresponding monodromy homomorphisms $h_{k}: \pi_{1}\left(S, s_{0}\right) \rightarrow G$ converge to $h_{\infty}$ within $\operatorname{Hom}\left(\pi_{1}\left(S, s_{0}\right), G\right)$.

We will be using Theorem [19. Fix an $\epsilon>0$. As in the prior proof we will only bother to choose a subsurface when $t \in\left[t_{k}-\epsilon, t_{k}+\epsilon\right]$ for some $k$. We will now describe how we choose these subsurfaces. As in the prior proof, we can let $L \subset S$ be a compact connected subsurface with boundary whose area is more than $1-\eta$ times the area of $S$ so that $L$ contains no zeros of $\alpha$. A compact surface with boundary has finite genus, so by removing small open neighborhoods of a maximal collection of smooth disjoint arcs joining $\partial L$ to itself whose collective complement in $L$ is connected, we may assume that $L$ has all these properties and is a homeomorphic to a closed topological disk. We can also assume by possibly adding a bit to the subsurface that $s_{0} \in L$. From above $(\tilde{S}, \tilde{\alpha})$ is translation equivalent to $g^{-t_{k}} b_{k}\left(\tilde{S}_{k}, \tilde{\alpha}_{k}\right)$ and so these surfaces cover $g^{-t_{k}} b_{k}(S, \alpha)$ by Proposition 10 . Let $L_{k} \subset\left(\tilde{S}_{k}, \tilde{\alpha}_{k}\right)$ be the collection of all lifts of $L$ under the covering map to $S$. The components of $L_{k}$ are naturally labeled $L_{k}^{1}, \ldots, L_{k}^{d}$ so that the lift of the basepoint $s_{k}^{i}$ lies in $L_{k}^{i}$ for all $i \in\{1, \ldots, d\}$. For $t \in\left[t_{k}-\epsilon, t_{k}+\epsilon\right]$ and $i \in\{1, \ldots, d\}$ we define $S_{t}^{i} \subset \tilde{S}$ to be image of $g^{-t_{k}} b_{k}\left(L_{k}^{i}\right)$ under a translation isomorphism $g^{-t_{k}} b_{k}\left(\tilde{S}_{k}, \tilde{\alpha}_{k}\right) \rightarrow(\tilde{S}, \tilde{\alpha})$. We define $S_{t}=\bigcup_{i=1}^{d} S_{t}^{i}$. Observe that with this definition: 
- The surface $S_{t}$ has a number of components $C_{t}$ equal to the degree $d$ of the covering maps.

Consider geometric quantities of $S_{t} \subset \tilde{S}$ measured with dist $t_{t}$ The surface $\tilde{S}$ with this metric is isometric to $g^{t}(\tilde{S}, \tilde{\alpha})$ which is translation equivalent to $g^{t-t_{k}} b_{k}\left(\tilde{S}_{k}, \tilde{\alpha}_{k}\right)$. So by definition of $S_{t}$ these geometric quantities are the same as for the subsurface $g^{t-t_{k}} b_{k}\left(L_{k}\right)$ of $g^{t-t_{k}} b_{k}\left(\tilde{S}_{k}, \tilde{\alpha}_{k}\right)$. Observe that $g^{t-t_{k}} b_{k}$ lies in the compact set $g^{[-\epsilon, \epsilon]} K$ where $K=\left\{b_{k}: k \in \mathbb{N}\right\} \cup\{b\}$ is compact as in the prior proof. Observe:

- The quantities $\epsilon(t)$ and $\mathcal{D}_{t}^{i}$ used to measure the components of $g^{t-t_{k}} b_{k}\left(L_{k}\right)$ as a subsurface of $g^{t-t_{k}} b_{k}\left(\tilde{S}_{k}, \tilde{\alpha}_{k}\right)$ are exactly the same as the quantities for $g^{t-t_{k}} b_{k}(L)$ viewed as a subsurface of $g^{t-t_{k}} b_{k}(S, \tilde{\alpha})$, because these quantities are covering map invariant.

In particular, this means that $\varepsilon(t)$ can be bounded uniformly away from zero when $t \in$ $\bigcup_{k}\left[t_{k}-\epsilon, t_{k}+\epsilon\right]$ and $\mathcal{D}_{t}^{i}$ can be bounded uniformly away from $+\infty$ as in the proof of Corollary 20.

It remains to control the quantity $\delta_{t}$. We must first construct the curves $\Gamma_{t}^{i, j}$. We utilize the convergence of $h_{k}$ to $h_{\infty}$. Since $S_{\infty}$ is connected, we can select for all distinct $i, j \in\{1, \ldots, d\}$ a path $\Gamma_{\infty}^{i, j}$ in $\tilde{S}_{\infty}$ disjoint from the zeros joining $s_{\infty}^{i}$ to $s_{\infty}^{j}$. Let $\tilde{\delta}_{0}>0$ be the minimum over all pair $(i, j)$ of the distance from $\Gamma_{\infty}^{i, j}$ to the set of points of the completion of $\tilde{S}$ which are either zeros or added in the completion. Let $\gamma^{i, j}$ be the loop in $S$ based at $s_{0}$ which is obtained as the image of $\Gamma_{\infty}^{i, j}$ under the covering map $\tilde{S}_{\infty} \rightarrow S$. Since $h_{k}$ tends to $h_{\infty}$, for $k$ sufficiently large $h_{k}\left(\gamma^{i, j}\right)=h_{\infty}\left(\gamma^{i, j}\right)$ for all $i$ and $j$. We will assume by dropping finitely many $k$ that this holds for all $k$. Then for each $k$, the lift $\tilde{\gamma}_{k}^{i, j}$ of $\gamma^{i, j}$ to $\tilde{S}_{k}$ which begins at $s_{k}^{i}$ ends at $s_{k}^{j}$. Observe that the minimal distance over all pairs $(i, j)$ of the distance of $\gamma^{i, j}$ to zeros or points added in the completion is still $\tilde{\delta}_{0}$. A subpath $\tilde{p}_{k}^{i, j} \subset \tilde{\gamma}_{k}^{i, j}$ joins $\partial L_{k}^{i}$ to $\partial L_{k}^{j}$ since the path joins $s_{k}^{i} \in L_{k}^{i}$ to $s_{k}^{j} \in L_{k}^{j}$. For $t \in\left[t_{k}-\epsilon, t_{k}+\epsilon\right]$, we define $\Gamma_{t}^{i, j} \subset S$ to be the image of $g^{-t_{k}} b_{k}\left(\tilde{p}_{k}^{i, j}\right)$ under the translation isomorphism $g^{-t_{k}} b_{k}\left(\tilde{S}_{k}, \tilde{\alpha}_{k}\right) \rightarrow(\tilde{S}, \tilde{\alpha})$ (as used

above to define $S_{t}$ ). The quantity $\delta_{t}$ is the minimal distance of $\Gamma_{t}^{i, j}$ to $\Sigma \subset \bar{S}$ taken over pairs $(i, j)$ and measured with dist $t_{t}$. As above $S$ with metric dist $t_{t}$ is isometric to $g^{t}(\tilde{S}, \tilde{\alpha})$ which in turn is translation isomorphic to $g^{t-t_{k}} b_{k}\left(\tilde{S}_{k}, \tilde{\alpha}_{k}\right)$. Thus we get the same value of $\delta_{t}$ by looking at the path $g^{t-t_{k}} b_{k}\left(\tilde{p}_{k}^{i, j}\right)$ in the translation surface $g^{t-t_{k}} b_{k}\left(\tilde{S}_{k}, \tilde{\alpha}_{k}\right)$. Thus $\delta_{t}$ is bounded from below by $\frac{1}{z} \tilde{\delta}_{0}$ when $t \in \bigcup_{k}\left[t_{k}-\epsilon, t_{k}+\epsilon\right]$ where $z$ is the maximal operator norm of $m^{-1}$ taken over $m$ taken from the compact set $g^{[-\epsilon, \epsilon]} K$ as above.

We have shown that the quantity being integrated in (14) has a positive lower bound when $t \in \bigcup_{k}\left[t_{k}-\epsilon, t_{k}+\epsilon\right]$. Since $t_{k} \rightarrow+\infty$, this means that the integral is infinite, so Theorem 19 guarantees that the translation flow is defined for all time almost everywhere and is ergodic.

\section{Further PROOFS}

In this section, we prove Proposition 2, Proposition 4 and Theorem 5 from the introduction.

Proof of Proposition 2 (Ergodicity and unique lifts of measures). Let $(S, \alpha)$ be a translation surface, let $(\tilde{S}, \tilde{\alpha})$ be a degree $d$ cover, and let $p: \tilde{S} \rightarrow S$ denote the covering map. We assume that the translation flow is ergodic on both of these surfaces. We will prove that Lebesgue measure is the unique translation flow invariant measure which projects to Lebesgue measure on $(S, \alpha)$ under the covering map. 
Fix a non-singular basepoint $s \in S$, and let $h: \pi_{1}(S, s) \rightarrow \Pi_{d}$ be the monodromy representation. We will consider the regular (or normal) cover of $S$ associated to the subgroup ker $h \subset \pi_{1}(S, s)$. Let $(\hat{S}, \hat{\alpha})$ denote this cover. Because the subgroup ker $h$ is normal, there is a covering group action of $\Delta=\pi_{1}(S, s) / \operatorname{ker} h$ on $\hat{S}$, and the quotient $\hat{S} / \Delta$ is naturally identified with $S$. The covering $\hat{S} \rightarrow S$ factors through $\tilde{S}$. That is, there is a subgroup $\Gamma \subset \Delta$ so that the $\tilde{S}$ is isomorphic as a cover to $\hat{S} / \Gamma$. We let $\hat{p}: \hat{S} \rightarrow \tilde{S}$ denote the covering obtained by identifying $\tilde{S}$ with $\hat{S} / \Gamma$.

Now suppose that $\tilde{\mu}$ is a measure on $\tilde{S}$ which is invariant for the translation flow, and satisfies $p_{*}(\tilde{\mu})=\lambda$, where $\lambda$ denotes Lebesgue measure on $(S, \alpha)$. Note that the measure $\tilde{\mu}$ lifts to a unique measure $\hat{\mu}$ on $(\hat{S}, \hat{\alpha})$ so that $\hat{p}_{*}(\hat{\mu})=\tilde{\mu}$ and so that $\gamma_{*}(\hat{\mu})=\hat{\mu}$ for all $\gamma \in \Gamma$. The key point in the proof is that because $\hat{\mu}$ projects through to Lebesgue measure on $S$, we know that if we average the push forwards of $\hat{\mu}$ under the covering group $\Delta$, we get Lebesgue measure on $(\hat{S}, \hat{\alpha})$, which we denote by $\hat{\lambda}$. That is,

$$
\frac{1}{|\Delta|} \sum_{\delta \in \Delta} \delta_{*}(\hat{\mu})=\hat{\lambda}
$$

Now consider the push-forward of these measures under the covering map $\hat{p}$. Since $p_{*}(\hat{\mu})=\tilde{\mu}$, we see that

$$
\frac{1}{|\Delta|}\left(\tilde{\mu}+\sum_{\delta \in \Delta \backslash\{e\}} \hat{p}_{*} \circ \delta_{*}(\hat{\mu})\right)=\tilde{\lambda},
$$

where $\tilde{\lambda}$ is the Lebesgue measure on $(\tilde{S}, \tilde{\alpha})$. Finally, $\tilde{\lambda}$ is ergodic, so each of the probability measures in the above convex combination must equal $\tilde{\lambda}$. In particular, $\tilde{\mu}=\tilde{\lambda}$, which concludes the proof.

Proof of Proposition 4. We assume that $(S, \alpha)$ is a finite area translation surface with infinite topological type and that it has Teichmüller trajectory which is non-divergent in $\mathcal{O}(S, \alpha)$. By non-divergence, there is a subsequence of times $t_{n} \rightarrow \infty$ so that $g^{t_{n}}(S, \alpha)$ tends to some $A_{\infty}(S, \alpha) \in \mathcal{O}(S, \alpha)$, where $A_{\infty} \in S L_{ \pm}(2, \mathbb{R})$. Because the topology on $\mathcal{O}(S, \alpha)$ arises as a quotient of the topology on $S L_{ \pm}(2, \mathbb{R})$, we see that there is a sequence $A_{n} \in S L(2, \mathbb{R})$ tending to $A_{\infty}$ so that $g^{t_{n}}(S, \alpha)$ is translation equivalent to $A_{n}(S, \alpha)$. It then follows that there is a sequence of elements $R_{n}$ of the Veech group of $(S, \alpha)$ so that $g^{t_{n}}=A_{n} R_{n}$.

Now consider a cover $(\tilde{S}, \tilde{\alpha})$ with monodromy in $G$. Proposition 10 explains that the Veech group acts on the space of covers with monodromy in $G$. In particular, for each $n$, there is a cover $\left(\tilde{S}_{n}, \tilde{\alpha}_{n}\right) \in \operatorname{Cov}_{G}(S, \alpha)$ which is translation equivalent to $R_{n}(\tilde{S}, \tilde{\alpha})$. Then, in the space $\tilde{\mathcal{O}}_{G}(S, \alpha)$, we have that

$$
g^{t_{n}}(\tilde{S}, \tilde{\alpha})=A_{n} R_{n}(\tilde{S}, \tilde{\alpha})=A_{n}\left(\tilde{S}_{n}, \tilde{\alpha}_{n}\right)
$$

The key observation is that $\operatorname{Cov}_{G}(S, \alpha)$ is a quotient of a Cantor set and thus sequentially compact, so there must be a limit point $\left(\tilde{S}_{\infty}, \tilde{\alpha}_{\infty}\right)$ for the sequence $\left(\tilde{S}_{n}, \tilde{\alpha}_{n}\right) \in \operatorname{Cov}_{G}(S, \alpha)$. Since $A_{n}$ tends to $A_{\infty}$ in $S L_{ \pm}(2, \mathbb{R})$, we see that $g^{t_{n}}(\tilde{S}, \tilde{\alpha})$ tends to $A_{\infty}\left(\tilde{S}_{\infty}, \tilde{\alpha}_{\infty}\right)$, which is our desired accumulation point.

Proof of Theorem 5 (Random covers accumulate on connected covers). Let $(S, \alpha)$ be a finite area translation surface with infinite topological type and a Teichmüller trajectory which is non-divergent in $\mathcal{O}(S, \alpha)$. As in the previous proof, this guarantees that there is a sequence 
$t_{n} \rightarrow \infty$ so that $g^{t_{n}}=A_{n} R_{n}$ where $\left\{A_{n} \in S L_{ \pm}(2, \mathbb{R})\right\}$ is a sequence tending to $A_{\infty} \in$ $S L_{ \pm}(2, \mathbb{R})$, and $R_{n} \in V(S, \alpha)$.

We will work with the space $\operatorname{TopCov}_{G}(S)$ of topological covers of $(S, \alpha)$ which comes equipped with a measure $\nu_{G}$. In order to do this, observe that for each Veech group element, $R_{n} \in V(S, \alpha)$ we can find an affine homeomorphism $\phi_{n}:(S, \alpha) \rightarrow(S, \alpha)$ so that $D\left(\phi_{n}\right)=R_{n}$. Recall that we denote elements of $\operatorname{Top} \operatorname{Cov}_{G}(S)$ by pairs $(p, \tilde{S})$ where $p: \tilde{S} \rightarrow S$ is a covering map. With an additional choice of a curve for each $n$, we obtain from $\phi_{n}$ an action

$$
\Phi_{n}: \operatorname{TopCov}_{G}(S) \rightarrow \operatorname{TopCov}_{G}(S)
$$

as in equation 9. Let $\mathcal{T}: \operatorname{Top} \operatorname{Cov}_{G}(S) \rightarrow \operatorname{Cov}_{G}(S, \alpha)$ be the map defined in (7). Then for each $(p, \tilde{S}) \in \operatorname{Top} \operatorname{Cov}_{G}(S)$ we have that

$$
R_{n}(\tilde{S}, \tilde{\alpha})=\left(\tilde{S}_{n}, \tilde{\alpha}_{n}\right) \quad \text { where }(\tilde{S}, \tilde{\alpha})=\mathcal{T}(p, \tilde{S}) \text { and }\left(\tilde{S}_{n}, \tilde{\alpha}_{n}\right)=\mathcal{T} \circ \Phi_{n}(p, \tilde{S}) .
$$

This follows from Proposition 10 and equation 9. In particular, with these hypotheses, we have that $g^{t_{n}}(\tilde{S}, \tilde{\alpha})$ is translation equivalent to $A_{n}\left(\tilde{S}_{n}, \tilde{\alpha}_{n}\right)$. Since the sequence $\left\{A_{n}\right\}$ converges in $S L_{ \pm}(2, \mathbb{R})$, it suffices to find a connected accumulation point of the sequence $\left\{\Phi_{n}(p, \tilde{S})\right\}$ for $\nu_{G}$-almost every $(p, \tilde{S}) \in \operatorname{TopCov}_{G}(S)$. (This is because our measure $m_{G}$ on the space of covers up to translation equivalence, $\operatorname{Cov}_{G}(S, \alpha)$, is the push forward of $\nu_{G}$ under the projection $\operatorname{TopCov}_{G}(S) \rightarrow \operatorname{Cov}_{G}(S, \alpha)$.)

Let $\mathcal{D} \subset \operatorname{Top}^{-\operatorname{Cov}_{G}}(S)$ denote the collection of disconnected surfaces. Note that these surfaces have $\nu_{G}$-measure zero by Proposition 18 . Let $\mathcal{E} \subset \operatorname{Top}_{\operatorname{Cov}_{G}}(S)$ denote the collection of covers $(p, \tilde{S}) \in \operatorname{Top}_{\operatorname{Cov}_{G}}(S)$ so that every accumulation point of $\left\{\Phi_{n}(p, \tilde{S})\right\}$ is disconnected. Then if $\mathcal{U} \subset \operatorname{Top}_{\operatorname{Cov}_{G}}(S)$ is open and contains $\mathcal{D}$, we have that for every $(p, \tilde{S}) \in \mathcal{E}$, there must be an $N$ so that $\Phi_{n}(p, \tilde{S}) \in \mathcal{U}$ for all $n>N$. Indeed, if this were not true, then infinitely many $\Phi_{n}(p, \tilde{S})$ lie in the complement of $\mathcal{U}$, which is sequentially compact since $\operatorname{Cov}_{G}(S, \alpha)$ is a Cantor set by Proposition 8. In other words, we have

$$
\mathcal{E} \subset \bigcup_{N} \bigcap_{n>N} \Phi_{n}^{-1}(\mathcal{U})=\liminf _{n \rightarrow \infty} \Phi_{n}^{-1}(\mathcal{U})
$$

Now fix some $\epsilon>0$. We will show that $\nu_{G}(\mathcal{E})<\epsilon$. Because $\nu_{G}$ is a Borel probability measure on a Cantor set, $\nu_{G}$ is regular. Thus because $\nu_{G}(\mathcal{D})=0$, we can find an open set $\mathcal{U}$ containing $\mathcal{D}$ so that $\nu_{G}(\mathcal{U})<\epsilon$. Then from the above, we have

$$
\nu_{G}(\mathcal{E}) \leq \nu_{G}\left(\liminf _{n \rightarrow \infty} \Phi_{n}^{-1}(\mathcal{U})\right) \leq \liminf _{n \rightarrow \infty} \nu_{G} \circ \Phi_{n}^{-1}(\mathcal{U}) .
$$

But, Corollary 15 tells us that $\nu_{G}$ is $\Phi_{n}$-invariant. Thus, the above sequence of inequalities tells us that $\nu_{G}(\mathcal{E}) \leq \nu_{G}(\mathcal{U})<\epsilon$. Since $\epsilon$ was arbitrary, we conclude that $\nu_{G}(\mathcal{E})=0$ as desired.

\section{ExAmples OF DeVious COVERS}

7.1. Chamanara's surfaces. We introduce a surface $\left(S_{2}, \alpha_{2}\right)$ first studied by Chamanara in Cha04. (See also the related work [CGL06].) The surface is built from a closed $1 \times 1$ square with each of the edges subdivided into intervals of length $\frac{1}{2^{k}}$ for $k \in \mathbb{N}$ as indicated in left side of Figure 1. The vertical intervals of equal length are then glued together by translation, and we do the same to the horizontal intervals. The intervals being identified have been labeled by the same integers in the figure. The endpoints of these intervals being glued and the corners of the square are discarded to give the space a translation structure. 


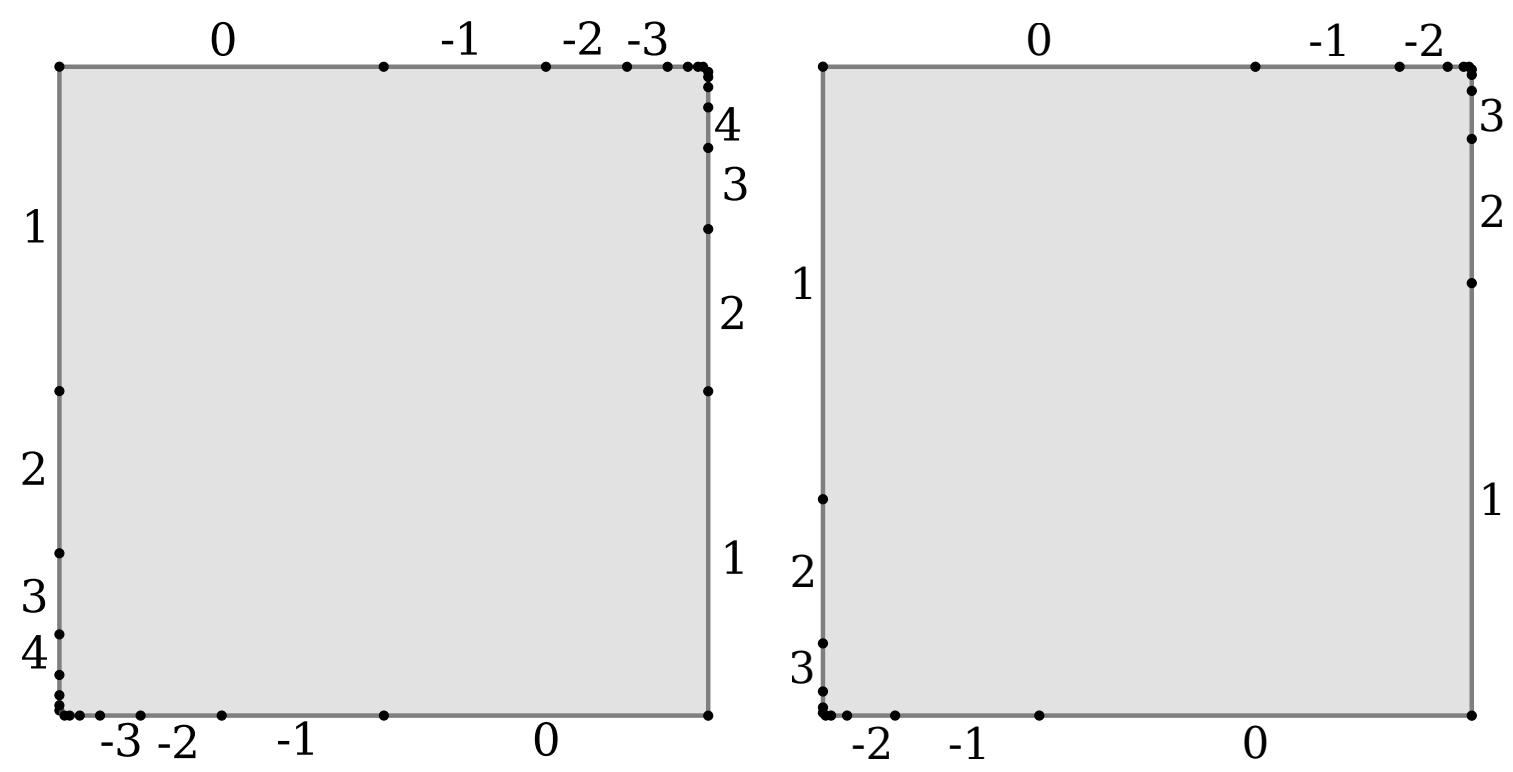

Figure 1. Chamanara's surfaces $\left(S_{2}, \alpha_{2}\right)$ and $\left(S_{3}, \alpha_{3}\right)$.

The surface $\left(S_{2}, \alpha_{2}\right)$ has an affine automorphism $\phi_{2}$ whose derivative is

$$
D\left(\phi_{2}\right)=\left[\begin{array}{ll}
\frac{1}{2} & 0 \\
0 & 2
\end{array}\right] \in V\left(S_{2}, \alpha_{2}\right) .
$$

To see this observe that the image of the square under this linear map is a $\frac{1}{2} \times 2$ rectangle. If we push this rectangle into the surface allowing the rectangle only to pass through the edge labeled zero in the figure, we see the identifications are respected and thus this map determines an affine automorphism $\phi_{2}$ of the surface.

The vertical and horizontal flows on Chamanara's surface $\left(S_{2}, \alpha_{2}\right)$ can be seen as the suspension flow over the dyadic odometer as defined in (3), except that our construction introduces countably many singularities through which the flows are not defined. To see this, it suffices to consider the dyadic odometer as an infinite interval exchange map on $[0,1]$ defined for $x \in[0,1]$, as the dyadic odometer on the dyadic expansion $\left(x_{1}, x_{2}, \ldots\right) \in X_{2}=$ $\{0,1\}^{\mathbb{N}}$ of $x=\sum x_{i} 2^{-i}$. As such, the map induced on a transversal $T_{2}$ running from the bottom to the top of the square making up $\left(S_{2}, \alpha_{2}\right)$ such as the one depicted in Figure 2 is isomorphic to the dyadic odometer. See [LTn16, §2] for a thorough description of the dyadic odometer and its relationship to Chamanara's surface.

More generally, for integers $n \geq 2$, one can construct a homeomorphic translation surface $\left(S_{n}, \alpha_{n}\right)$ by letting the identified sides in Figure 1 be of length $\frac{n-1}{n^{k}}$ for $k \in \mathbb{N}$. The right side of Figure 1 illustrates the case of $n=3$. The surface $\left(S_{n}, \alpha_{n}\right)$ admits an affine automorphisms $\phi_{n}$ with diagonal derivative and eigenvalues of $n$ and $\frac{1}{n}$. As in the case of the dyadic odometer, this surface admits a section which is the $n$-adic odometer.

Remark 21 (Veech groups). The Veech group $V\left(S_{n}, \alpha_{n}\right)$ is known to be generated by two parabolics. See [HR16].

We will now introduce some more notation which will be useful for the Proof of Theorem 7 and for work later in this section. Let $G$ be a subgroup of the symmetric group $\Pi_{d}$ with $d \geq 2$ which acts simply transitively on $\{1, \ldots, d\}$. For a general surface $(S, \alpha)$ the space 
$\operatorname{Cov}_{G}(S, \alpha)$ of covers with monodromy in $G$ is a quotient of the space of topological covers $\operatorname{TopCov}_{G}(S)$ by the translation equivalence relation; see (7). However it is not hard to see that by Remark 9 that this equivalence relation is trivial in the case of $\left(S_{n}, \alpha_{n}\right)$. (The surface $\left(S_{n}, \alpha_{n}\right)$ is formed by identifying the boundary edges of the square along an IET. The deck group of the universal cover of $\left(S_{n}, \alpha_{n}\right)$ acts transitively on lifts of these squares, and the collection of these lifts must be preserved by translation automorphisms because of the singularities in their boundary.) Thus by recalling (6) we see that the space of covers of $\left(S_{n}, \alpha_{n}\right)$ with monodromy in $G$ can be thought of as

$$
\operatorname{Cov}_{G}\left(S_{n}, \alpha_{n}\right)=\Pi_{d} \backslash \operatorname{Hom}\left(\pi_{1}\left(S_{n}, s_{0}\right), G\right),
$$

where $s_{0}$ is a basepoint of $S_{n}$. If $h$ is a homomorphism from $\pi_{1}\left(S_{n}, s_{0}\right)$ to $G$, we use $[h]$ to denote its equivalence class in $\operatorname{Cov}_{G}\left(S_{n}, \alpha_{n}\right)$.

Recall that formally, the affine automorphism $\phi_{n}^{-1}$ does not act on the fundamental group of $S_{n}$. We choose a basepoint $s_{0}$ in the interior of the square near the southwest corner of the square in Figure 1. To get an action on the fundamental group, we need to select a curve joining $s_{0}$ to $\phi_{n}^{-1}\left(s_{0}\right)$ as described by equation 8 . Because we chose $s_{0}$ in the interior of the square near the southwest corner, its image $\phi_{n}^{-1}\left(s_{0}\right)$ will also lie near the southwest corner and in the interior of the square. We specify $\beta$ to be a curve joining $\phi^{-1}\left(s_{0}\right)$ to $s_{0}$ while not leaving the interior of the square. Then as in (8) we define the group automorphism

$$
\phi_{\beta}^{-1}: \pi_{1}\left(S_{n}, s_{0}\right) \rightarrow \pi_{1}\left(S_{n}, s_{0}\right) ; \quad[\gamma] \mapsto\left[\beta^{-1} \bullet\left(\phi_{n}^{-1} \circ \gamma\right) \bullet \beta\right] .
$$

We now see that Theorem 7 is a consequence of Corollary 6 .

Proof of Theorem 7 . Recall that the $n$-adic odometer is uniquely ergodic, because it can be understood as a minimal rotation of a compact abelian group. Therefore, the translation flow on the surface $\left(S_{n}, \alpha_{n}\right)$ is also uniquely ergodic. Observe that the Teichmüller flow is periodic, because the affine automorphism $\phi_{n}$ has diagonal derivative.

We begin with some definitions which are illustrated in Figure 2. Since this figure illustrates $n=2$, we will just discuss this case for this paragraph. The dotted line $T_{2}$ on $S_{2}$, isometric to $[0,1]$, is a transversal to the translation flow. We selected this transversal to be vertical and to pass through the basepoint $s_{0}$ of $S_{2}$. The return map to the transversal is isomorphic to the dyadic odometer. We create an infinite set of closed loops $\gamma_{i}$ on $S_{2}$ indexed by $\mathbb{N}$. For each $i \in \mathbb{N}$, the loop $\gamma_{i}$ moves from the basepoint to a point $x \in T_{2}$ within $T_{2}$, follows the translation flow until it first returns to $T_{2}$, and then travels back to the basepoint within $T_{2}$ (shown in Figure 2 as three dashed line segments). In defining $\gamma_{i}$, we insist that the dyadic expansion $\left(x_{1}, x_{2}, x_{3}, \ldots\right)$ of $x$ has the property that $x_{j}=1$ for all $j<i$ and $x_{i}=0$. Doing this for every $i \in \mathbb{N}$ we create the countable set $\left\{\gamma_{i}\right\}$. Let $\Gamma^{+}=\left\langle\gamma_{1}, \gamma_{2}, \ldots\right\rangle$ be the free group generated by the $\gamma_{i}$ which is a subgroup of $\Gamma=\pi_{1}\left(S_{2}, s_{0}\right)$.

The general case is not qualitatively different. Following the same ideas in the previous paragraph, we define $\gamma_{i}, \Gamma^{+}$and $\Gamma$ for all integers $n \geq 2$.

Fix a $d \geq 2$ and a subgroup $G \subset \Pi_{d}$ that acts transitively on $\{1, \ldots, d\}$. Corollaries 3 and 6 imply that $m_{G}$-almost every cover has a uniquely ergodic translation flow, where $m_{G}$ is the probability measure on $\operatorname{Cov}_{G}\left(S_{n}, \alpha_{n}\right)$ from $\$ 4.2$. Recall that $m_{G}$ is the measure induced by the quotient (17) from the product measure $\mu$ on $\operatorname{Hom}(\Gamma, G)$; see Definition 12 of in $\S 4.2$. Thus, for $\mu$-a.e. $h \in \operatorname{Hom}(\Gamma, G)$, the corresponding cover $\left(\tilde{S}_{h}, \tilde{\alpha}_{h}\right)$ has uniquely ergodic translation flow. 

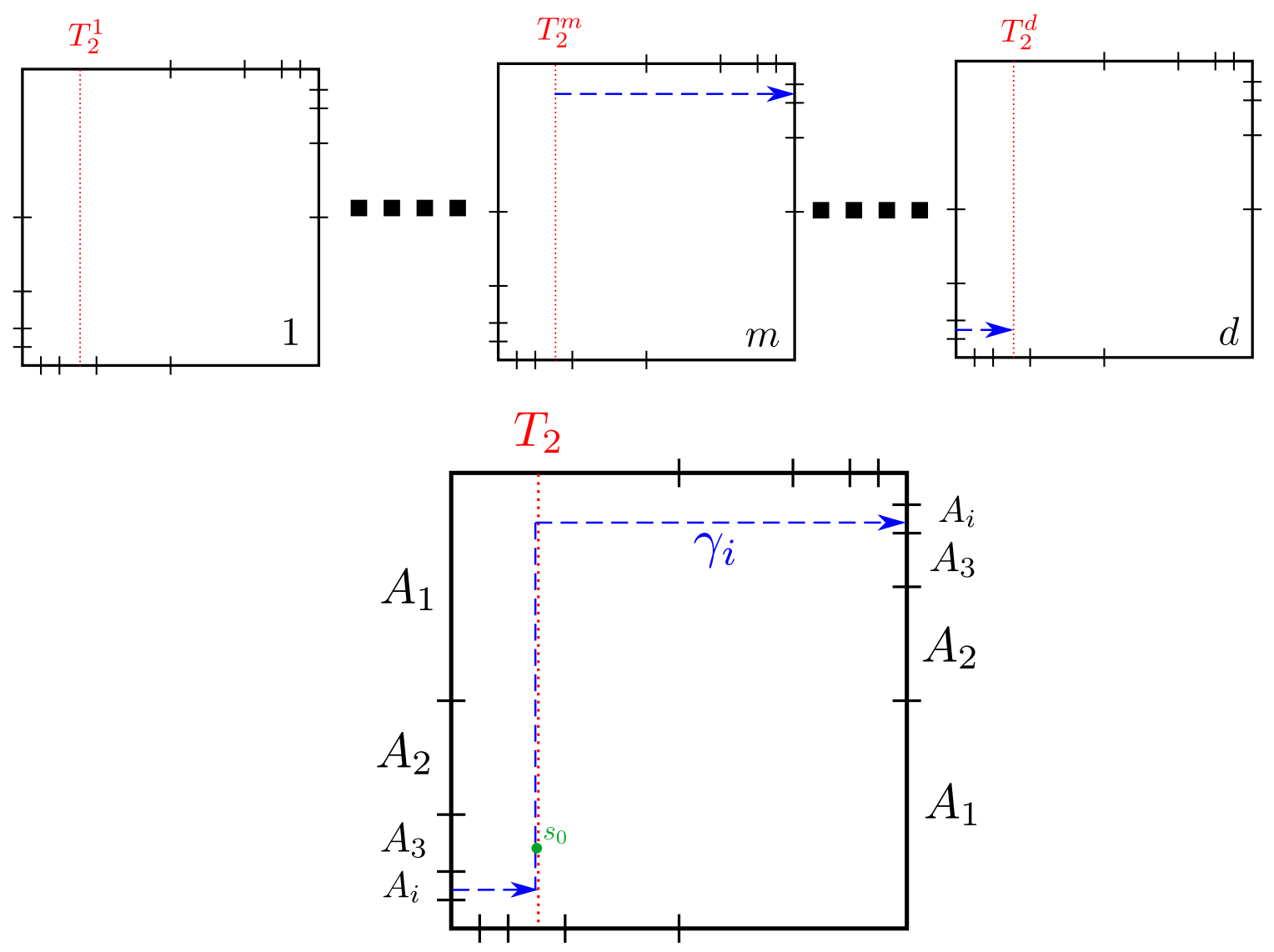

Figure 2. Bottom: The surface $\left(S_{2}, \alpha_{2}\right)$ with the transversal $T_{2}$ depicted with a dotted line, and a curve $\gamma_{i}$ shown as a dashed line. Top: A $d$-fold cover $\left(\tilde{S}_{\psi}, \tilde{\alpha}_{\psi}\right)$ is built out of copies of the square labeled $\{1, \ldots, d\}$ with edges glued according to $\psi_{+}$. The dotted lines are the lifts of the transversals and the dashed is a lift of the horizontal part of the path of $\gamma_{i}$ depicted under the assumption that $\psi\left(\gamma_{i}\right)(m)=d$.

Consider the automorphism $\phi_{\beta}^{-1}$ of $\pi_{1}\left(S_{n}, \alpha_{n}\right)$ given in 18$)$. We observe by inspecting the action of $\phi^{-1}$ that

$$
\phi_{\beta}^{-1}\left(\gamma_{i}\right)=\gamma_{1}^{n-1} \gamma_{i+1} \quad \text { for all } i \geq 1,
$$

where calculations are done in the fundamental group. This implies that $\phi_{\beta}^{-1}\left(\Gamma^{+}\right) \subset \Gamma^{+}$. The inclusion $i: \Gamma^{+} \rightarrow \Gamma$ then induces a surjective map $i^{*}: \operatorname{Hom}(\Gamma, G) \rightarrow \operatorname{Hom}\left(\Gamma^{+}, G\right)$ which commutes with the actions of $\phi_{\beta}^{-1}$. The set $\operatorname{Hom}\left(\Gamma^{+}, G\right)$ is a Cantor set with a product measure $\mu_{+}$as defined in Definition 12. It is straight forward to see from the definitions of these measures that $\mu_{+}=\left(i^{*}\right)_{*} \mu$.

Let $i^{*}(\psi)=\psi_{+} \in \operatorname{Hom}\left(\Gamma^{+}, G\right)$ for some $\psi \in \operatorname{Hom}(\Gamma, G)$ and let $\left(\tilde{S}_{\psi}, \tilde{\alpha}_{\psi}\right)$ be the $d$-cover of $\left(S_{n}, \alpha_{n}\right)$ determined by $\psi$. The transversal $T_{2}$ lifts to $\tilde{T}_{2}$, a transversal on $\tilde{S}_{\psi}$ for the translation flow. It is the union of the $d$ copies $T_{2}^{1}, \ldots, T_{2}^{d}$ of $T_{2}$, which are also illustrated using dotted lines in the figure. As such, the translation flow on a cover $\left(\tilde{S}_{\psi}, \tilde{\alpha}_{\psi}\right)$ is canonically a suspension flow of the skew product over the $n$-adic odometer. One can observe that this skew product is precisely $E_{\psi_{+}}$given in (4) of the introduction. Whenever the translation 
flow on $\left(\tilde{S}_{\psi}, \tilde{\alpha}_{\psi}\right)$ is uniquely ergodic, the skew product $E_{\psi_{+}}$must be as well. Since this holds for $\mu$ a.e. $\psi$ and $\mu_{+}=\left(i^{*}\right)_{*} \mu$, we see that for $\mu_{+}$a.e. $\psi_{+}, E_{\psi_{+}}$is uniquely ergodic.

Pathological covers. For the remainder of the section, we will concentrate on the simplest of Chamanara's surfaces, $\left(S_{2}, \alpha_{2}\right)$. We will simplify the notation for the hyperbolic automorphism $\phi_{2}$ by denoting it by $\phi$.

Our goal with the remainder of this section is to investigate what happens when we have a connected cover $\left(\tilde{S}_{2}, \tilde{\alpha}_{2}\right)$ which when iterated by application of $\phi$ only accumulates on disconnected covers. The disconnected covers in $\operatorname{Cov}_{G}\left(S_{n}, \alpha_{n}\right)$ are given by

$$
\bigcup_{H \in \mathcal{H}} \Pi_{d} \backslash \operatorname{Hom}(\Gamma, H)
$$

where $\Gamma=\pi_{1}\left(S_{2}, s_{0}\right)$ and $\mathcal{H}$ denotes the collection of all subgroups of $G$ which fail to act transitively on $\{1, \ldots, d\}$.

It can be observed that the connectivity of $(\tilde{S}, \tilde{\alpha})$ does not guarantee the ergodicity of the (horizontal) translation flow, since the connectivity can be arranged with only the gluings of horizontal edges when building the cover as $d$ copies of the unit square with edge identifications. It is not surprising then that such connected devious covers are dense inside of the space of covers:

Theorem 22 (Non-ergodic covers). Let $h \in \operatorname{Hom}(\Gamma, G)$. The translation flow on the cover $\left(\tilde{S}_{h}, \tilde{\alpha}_{h}\right)$ of $\left(S_{2}, \alpha_{2}\right)$ associated to $[h]$ is non-ergodic whenever there is an $H \in \mathcal{H}$ so that every accumulation point of $h \circ \phi_{\beta}^{-n}$ (as $n \rightarrow+\infty$ ) lies in $\operatorname{Hom}(\Gamma, H)$. For any $H \in \mathcal{H}$, there exists a collection of connected covers dense in $\operatorname{Cov}_{G}\left(S_{2}, \alpha_{2}\right)$ so that every accumulation point lies in $\Pi_{d} \backslash \operatorname{Hom}(\Gamma, H)$ but no accumulation point lies in $\Pi_{d} \backslash \operatorname{Hom}\left(\Gamma, H^{\prime}\right)$ for any proper subgroup $H^{\prime} \subset H$.

It is interesting to consider whether there are connected covers which accumulate only on disconnected covers under the Teichmüller deformation but whose translation flow is nonetheless uniquely ergodic. We show such covers exist. This is analogous to sufficiently slow divergence of the Teichmüller deformation giving rise to unique ergodicity in the classical setting of closed translation surfaces as in [CE07] [Tre14].

Theorem 23. Suppose $G$ is a subgroup of $\Pi_{d}$, and $H_{1}, H_{2} \subset G$ are subgroups which do not act transitively on $\{1, \ldots, d\}$, but the group generated by the elements of $H_{1} \cup H_{2}$ does act transitively. Then, there are finite covers of $\left(S_{2}, \alpha_{2}\right)$ with monodromy in $G$ whose translation flow is uniquely ergodic, but whose orbit under $\phi$ accumulates only on surfaces in the collection of disconnected covers,

$$
\Pi_{d} \backslash\left(\operatorname{Hom}\left(\Gamma, H_{1}\right) \cup \operatorname{Hom}\left(\Gamma, H_{2}\right)\right) .
$$

Remark 24. It seems likely that there are also non-ergodic covers whose orbits under $\phi$ accumulate as in Theorem 23. We do not investigate this question.

The key to proving these results is an understanding of the action on $\operatorname{Hom}(\Gamma, G)$ given by

$$
h \mapsto h \circ \phi_{\beta}^{-1},
$$

where $\phi_{\beta}^{-1}: \Gamma \rightarrow \Gamma$ is the automorphism from $(18)$.

In order to describe this action, we select a generating set for $\Gamma$. For each integer $n$, we will let $\gamma_{n} \in \Gamma$ be a homotopy class of curves which start and end at the basepoint. If $n \leq 0$, we 
define $\gamma_{n}$ to contain the curves which move downward from the basepoint passing through the horizontal edge labeled $n$ and returning to the basepoint without passing through any other labeled edges. We similarly define $\gamma_{n}$ for $n>0$ to contain the curves which move rightward over the vertical edge labeled $n$. (This is compatible with the definition of $\gamma_{n}$ for $n \geq 1$ in the Proof of Theorem 7, and depicted in Figure 2.) Observe:

$$
\Gamma=\pi_{1}\left(S_{2}, s_{0}\right) \quad \text { is freely generated by }\left\{\gamma_{n}: n \in \mathbb{Z}\right\} .
$$

(The curves $\gamma_{n}$ can be taken to be pairwise disjoint except at the basepoint and so the union of these curves is a bouquet of countably many circles. There is a deformation retraction of the surface to this bouquet.)

The dynamics of $\phi_{\beta}$ action on $\operatorname{Hom}(\Gamma, G)$ turn out to be conjugate to the shift on $G^{\mathbb{Z}}$ :

Lemma 25. The map $\mathfrak{g}: \operatorname{Hom}(\Gamma, G) \rightarrow G^{\mathbb{Z}}$ defined by

$$
\mathfrak{g}(h)_{m}=h \circ \phi_{\beta}^{-m}\left(\gamma_{1}\right)
$$

is a homeomorphism. Let $\sigma: G^{\mathbb{Z}} \rightarrow G^{\mathbb{Z}}$ be the shift map $\sigma(\mathbf{g})_{i}=\mathbf{g}_{i+1}$. Then

$$
\mathfrak{g}\left(h \circ \phi_{\beta}^{-1}\right)=\sigma \circ \mathfrak{g}(h) \quad \text { for all } h \in \operatorname{Hom}(\Gamma, G) .
$$

We call $\mathfrak{g}(h)$ the $G$-sequence of $h$. As a first step to proving this theorem, we work out the action of $\phi_{\beta}^{-1}$ and its inverse $\phi_{\beta}$ on $\Gamma$ :

Proposition 26. For each $n \in \mathbb{N}$, we have

$$
\phi_{\beta}^{-1}\left(\gamma_{n}\right)=\left\{\begin{array}{ll}
\gamma_{n+1} \gamma_{1}^{-1} & \text { if } n<0 \\
\gamma_{1} & \text { if } n=0 \\
\gamma_{1} \gamma_{n+1} & \text { if } n>0,
\end{array} \quad \text { and } \quad \phi_{\beta}\left(\gamma_{n}\right)= \begin{cases}\gamma_{n-1} \gamma_{0} & \text { if } n \leq 0 \\
\gamma_{0} & \text { if } n=1 \\
\gamma_{0}^{-1} \gamma_{n-1} & \text { if } n>1 .\end{cases}\right.
$$

This proposition may be proved by inspecting the action of $\phi_{\beta}^{-1}$ as defined in (18). We leave the details to the reader.

The following lemma describes how to recover information about the $\phi_{\beta}$-orbit of $h$ from its $G$-sequence.

Lemma 27. Let $\mathbf{g}=\mathfrak{g}(h)$ be the $G$-sequence of a homomorphisms $h: \Gamma \rightarrow G$. Then, for each $k, n \in \mathbb{Z}$,

$$
h \circ \phi_{\beta}^{-k}\left(\gamma_{n}\right)= \begin{cases}\mathbf{g}_{k+n-1} \mathbf{g}_{k+n}^{-1} \mathbf{g}_{k+n+1}^{-1} \ldots \mathbf{g}_{k-1}^{-1} & \text { if } n<0 \\ \mathbf{g}_{k-1} & \text { if } n=0 \\ \mathbf{g}_{k} & \text { if } n=1 \\ \mathbf{g}_{k}^{-1} \mathbf{g}_{k+1}^{-1} \ldots \mathbf{g}_{k+n-2}^{-1} \mathbf{g}_{k+n-1} & \text { if } n>1 .\end{cases}
$$

Proof. Observe that by definition $h \circ \phi_{\beta}^{-k}\left(\gamma_{1}\right)=\mathbf{g}_{k}$ for all $k \in \mathbb{Z}$. This case of $n=1$ will serve as a base case for proving the statement holds when $n \geq 1$. Note that the formula given in the case of $n>1$ can be extended to hold for $n=1$ if one allows the (empty) product of inverses $\mathbf{g}_{k}^{-1} \mathbf{g}_{k+1}^{-1} \ldots \mathbf{g}_{k+n-2}^{-1}$ to be the identity when $n=1$. So, suppose our formula holds for some $n \geq 1$ and all $k$, we will show it holds for $n+1$ and all $k$. Using the proposition, we observe that for $n \geq 1$,

$$
h \circ \phi_{\beta}^{-k-1}\left(\gamma_{n}\right)=h \circ \phi_{\beta}^{-k}\left(\gamma_{1} \gamma_{n+1}\right)=\mathbf{g}_{k} \cdot h \circ \phi_{\beta}^{-k}\left(\gamma_{n+1}\right) .
$$

By our inductive hypothesis applied to the left side, we see that

$$
\mathbf{g}_{k+1}^{-1} \mathbf{g}_{k+2}^{-1} \ldots \mathbf{g}_{k+n-1}^{-1} \mathbf{g}_{k+n}=\mathbf{g}_{k} \cdot h \circ \phi_{\beta}^{-k}\left(\gamma_{n+1}\right)
$$


which gives us that $\phi_{\beta}^{-k}\left(\gamma_{n+1}\right)=\mathbf{g}_{k}^{-1} \ldots \mathbf{g}_{k+n-1}^{-1} \mathbf{g}_{k+n}$. This proves our formula for $n+1$ and and all $k$. So, by induction, the statement holds for $n \geq 1$.

Now we consider the base case of $n=0$. Observe that

$$
h \circ \phi_{\beta}^{-k}\left(\gamma_{0}\right)=h \circ \phi_{\beta}^{-k+1} \circ \phi_{\beta}^{-1}\left(\gamma_{0}\right)=h \circ \phi_{\beta}^{-k+1}\left(\gamma_{1}\right)=\mathbf{g}_{k-1} .
$$

We will proceed by induction to cover cases with $n<0$. The case of $n=0$ can serve as our base case since when $n=0$ we have $\mathbf{g}_{k+n-1} \mathbf{g}_{k+n}^{-1} \mathbf{g}_{k+n+1}^{-1} \ldots \mathbf{g}_{k-1}^{-1}=\mathbf{g}_{k-1}$ by convention as above since the product of negations $\mathbf{g}_{k+n}^{-1} \mathbf{g}_{k+n+1}^{-1} \ldots \mathbf{g}_{k-1}^{-1}$ is an empty product (as $k+n>k-1$ here). Now suppose the formula holds for some $n \leq 0$ and all $k$. Then, by the proposition and the base case

$$
h \circ \phi_{\beta}^{-k+1}\left(\gamma_{n}\right)=h \circ \phi_{\beta}^{-k}\left(\gamma_{n-1} \gamma_{0}\right)=h \circ \phi_{\beta}^{-k}\left(\gamma_{n-1}\right) \cdot \mathbf{g}_{k-1} .
$$

By inductive hypothesis, we see

$$
h \circ \phi_{\beta}^{-k}\left(\gamma_{n-1}\right)=h \circ \phi_{\beta}^{-k+1}\left(\gamma_{n}\right) \cdot \mathbf{g}_{k-1}^{-1}=\left(\mathbf{g}_{k+n-2} \mathbf{g}_{k+n-1}^{-1} \ldots \mathbf{g}_{k}^{-1}\right) \mathbf{g}_{k-1}^{-1} .
$$

This completes the inductive step, proving the statement for all $n \leq 0$.

This Lemma allow us to prove that $\mathfrak{g}$ is a topological conjugacy:

Proof of Lemma 25. The map $\mathfrak{g}$ is clearly continuous. The identity provided in Lemma 27 considered in the special case of $k=0$ allows us to evaluate $h$ on the generators $\left\{\gamma_{n}\right\}$ of $\Gamma$ in terms of $\mathfrak{g}(h)$. This therefore gives an inverse $\mathfrak{g}^{-1}$, and we observe that it is continuous. The conjugacy equation is easily verified:

$$
\mathfrak{g}\left(h \circ \phi_{\beta}^{-1}\right)_{m}=h \circ \phi_{\beta}^{-1} \circ \phi_{\beta}^{-m}\left(\gamma_{1}\right)=h \circ \phi_{\beta}^{-m-1}\left(\gamma_{1}\right)=\mathfrak{g}(h)_{m+1}=(\sigma \circ \mathfrak{g}(h))_{m}
$$

for all $m \in \mathbb{Z}$.

Now we prove Theorem 22 on the non-ergodicity of covers.

Proof of Theorem 22. Let $h: \Gamma \rightarrow G$ be a homomorphism, and suppose that all accumulation points of $h \circ \phi_{\beta}^{-k}$ as $k \rightarrow \infty$ lie in $\operatorname{Hom}(\Gamma, H)$ for some subgroup $H \subset G$, where $H$ does not act transitively on $\{1, \ldots, d\}$. We will show that the translation flow on $\left(\tilde{S}_{h}, \tilde{\alpha}_{h}\right)$ is not ergodic. By compactness of $\operatorname{Hom}(\Gamma, H)$, there is a $K$ so that for each $k \geq K, h \circ \phi_{\beta}^{-k}\left(\gamma_{1}\right) \in H$. In terms of the $G$-sequence $\mathbf{g}=\mathfrak{g}(h)$, we see that $\mathbf{g}_{k} \in H$ for $k \geq K$. It can be observed from the formula in Lemma 27 that it follows that $h \circ \phi_{\beta}^{-K}\left(\gamma_{n}\right) \in H$ for all $n \geq 1$. Observe that the horizontal straight-line flow on the surface $\left(S_{2}, \alpha_{2}\right)$ only crosses the intervals with positive label in Figure 1. Now consider the cover associated to $h \circ \phi_{\beta}^{-K}$. The cover can be built from copies of the square indexed by $\{1, \ldots, d\}$ with edges identified according to $h \circ \phi_{\beta}^{-K}$. In particular, the intervals with positive label are glued only according to elements of $H$. Thus, points in copy $i \in\{1, \ldots, d\}$ only can reach the copies of the square indexed by elements of the orbit $H(i)$, and by assumption $H(i) \neq\{1, \ldots, d\}$. In particular the union of the squares indexed by $H(i)$ gives an invariant set with measure strictly between zero and full measure. Note that $\phi_{\beta}^{K}$ induces an affine homeomorphism with diagonal derivative from the cover associated to $h$ to the cover associated to $h \circ \phi_{\beta}^{-K}$, so pulling back this invariant set gives an invariant subset of the straight line flow for the cover associated to $h$ with intermediate measure as desired.

We will now construct a dense set of devious covers as described in second sentence of the Theorem. Consider the set $X_{H} \subset G^{\mathbb{Z}}$ consisting of those $\mathbf{g} \in G^{\mathbb{Z}}$ which satisfy the following statements: 
(1) The subgroup of $G$ generated by $\left\{\mathbf{g}_{k}: k \in \mathbb{Z}\right\}$ acts transitively on $\{1, \ldots, d\}$.

(2) There is a $K \in \mathbb{Z}$ so that $\mathbf{g}_{k} \in H$ for $k>K$.

(3) There is a $B>0$ so that for any $k>K$, the subgroup $H \subset G$ is generated by $\left\{\mathbf{g}_{k}, \mathbf{g}_{k+1}, \ldots, \mathbf{g}_{k+B-1}\right\}$.

It should be clear that $X_{H}$ is dense in $G^{\mathbb{Z}}$. Since $\mathfrak{g}$ is a homeomorphism by Lemma 25, we know $\mathfrak{g}^{-1}\left(X_{H}\right)$ is dense in $\operatorname{Hom}(\Gamma, G)$. Now let $h \in \mathfrak{g}^{-1}\left(X_{H}\right)$ and consider the cover $\left(\tilde{S}_{h}, \tilde{\alpha}_{h}\right)$ of $\left(S_{2}, \alpha_{2}\right)$. By Lemma 27 , the group generated by $\left\{\mathbf{g}_{k}\right\}$ coincides with the image $h(\Gamma)$ and so statement (1) implies that $\tilde{S}_{h}$ is connected. Now let $h^{\prime}$ be any accumulation point of $h \circ \phi_{\beta}^{-n}$ taken as $n \rightarrow+\infty$. Let $\mathbf{g}^{\prime}=\mathfrak{g}\left(h^{\prime}\right)$. Then by Lemma 25, $\mathbf{g}^{\prime}$ is an accumulation point of $\sigma^{n}(\mathbf{g})$. Statement (2) then implies that $\mathbf{g}^{\prime} \in H^{\mathbb{Z}}$ and so $h^{\prime} \in \operatorname{Hom}(\Gamma, H)$ by Lemma 27. Statement (3) implies that $\left\{\mathbf{g}_{1}^{\prime}, \mathbf{g}_{2}^{\prime}, \ldots, \mathbf{g}_{B}^{\prime}\right\}$ generates $H$ and Lemma 27 then guarantees that $h^{\prime}(\Gamma)=H$ so in particular $h^{\prime} \notin \operatorname{Hom}\left(\Gamma, H^{\prime}\right)$ for any proper subgroup $H^{\prime} \subset H$.

Now we will move toward proving Theorem 22 about the existence of covers with ergodic translation flow whose $\phi_{\beta}$-orbits accumulate only on disconnected covers. Recall that this theorem dealt with the situation when we have two subgroups $H_{1}$ and $H_{2}$ of $G$ which do not act transitively on $\{1, \ldots, d\}$ but which together act transitively.

We now introduce a combinatorial tool that will allow us to find examples of $\mathbf{g}=\mathfrak{g}(h)$ where the sequence of covers associated to $h \circ \phi_{\beta}^{-k}$ only has disconnected limits. Let $a \in \mathbb{Z}$ and $A=\{n \in \mathbb{Z}: n \geq a\}$. Let $p: A \rightarrow\{0,1,2\}$ be an arbitrary function with the property that $p(n)+p(n+1) \neq 3$ for each $n \in A$ (or equivalently $p(n)=1$ implies $p(n+1) \neq 2$ and $p(n)=2$ implies $p(n+1) \neq 1$.) For such a map $p$ and an $n \in A$, we define the preimage interval containing $n, I(p, n) \subset \mathbb{Z}$, to be the maximal collection of consecutive elements of $A$ so that $n \in I(p, n)$ and $p$ is constant on $I(p, n)$. We'll say that $\mathbf{g} \in G^{\mathbb{Z}}$ is $p$-ready if the following two statements hold for each $n \in A$ :

- If $p(n)=0$, then $\mathbf{g}_{n} \in H_{1} \cap H_{2}$.

- If $p(n) \in\{1,2\}$, then $\mathbf{g}_{n} \in H_{p(n)}$ and $\left\{\mathbf{g}_{k}: k \in I(p, n)\right\}$ generates $H_{p(n)}$.

Let $|I(p, n)|$ denote the number of integers in $I(p, n)$.

Proposition 28 (Criterion for disconnected accumulation points). Let $h \in \operatorname{Hom}(\Gamma, G)$ and suppose $\mathbf{g}=\mathfrak{g}(h)$ is p-ready. Suppose that

$$
\lim _{n \rightarrow \infty ; p(n)=0}|I(p, n)|=\infty,
$$

(with the limit taken only over those $n$ so that $p(n)=0$ ). Then every accumulation point of $h \circ \phi_{\beta}^{-k}$ as $k \rightarrow \infty$ corresponds to a disconnected cover. Moreover, these accumulation points all lie in $\operatorname{Hom}\left(\Gamma, H_{1}\right) \cup \operatorname{Hom}\left(\Gamma, H_{2}\right)$.

Proof. Let $h_{\infty}$ be an accumulation point of $h \circ \phi_{\beta}^{-k}$ as $k \rightarrow \infty$. Then there is an increasing sequence of integers $k_{j}$ so that $h_{\infty}=\lim _{j \rightarrow \infty} h \circ \phi_{\beta}^{-k_{j}}$. Let $\mathbf{g}^{\infty}=\mathfrak{g}\left(h_{\infty}\right)$. By Lemma 25, we have

$$
\mathbf{g}^{\infty}=\lim _{j \rightarrow \infty} \sigma^{k_{j}}(\mathbf{g}) .
$$

We claim there is an $i \in\{1,2\}$ so that $\mathbf{g}_{m}^{\infty} \in H_{i}$ for all $m \in \mathbb{Z}$. If not, there are two indices $n_{1}$ and $n_{2}$ so that $\mathbf{g}_{n_{1}}^{\infty} \in H_{1} \backslash H_{2}$ and $\mathbf{g}_{n_{2}}^{\infty} \in H_{2} \backslash H_{1}$. Then for $j$ large enough, $\sigma^{k_{j}}(\mathbf{g})_{n_{1}}=\mathbf{g}_{k_{j}+n_{1}}$ and $\sigma^{k_{j}}(\mathbf{g})_{n_{2}}=\mathbf{g}_{k_{j}+n_{2}}$ have the same property. From the definition of $p$-ready we have $p\left(k_{j}+n_{1}\right)=1$ and $p\left(k_{j}+n_{2}\right)=2$ so between $k_{j}+n_{1}$ and $k_{j}+n_{2}$ there is a $m_{j} \in \mathbb{Z}$ so that $p\left(m_{j}\right)=0$ and we must have $\left|I\left(p, m_{j}\right)\right|<\left|n_{1}-n_{2}\right|$. This violates (20). 


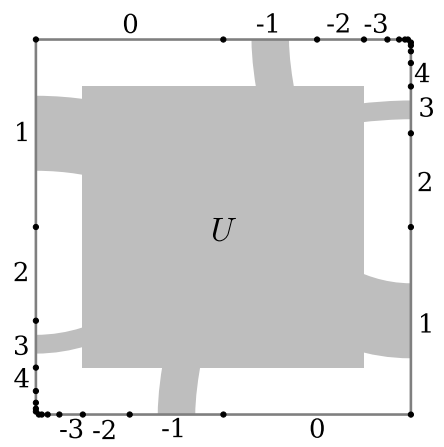

Figure 3. A subsurface $U$ when $J=\{-1,1,3\}$.

We have shown $\mathbf{g}^{\infty} \in H_{i}^{\mathbb{Z}}$. Lemma 27 implies that $h_{\infty} \in \operatorname{Hom}\left(\Gamma, H_{i}\right)$.

Our proof of Theorem 23 will combine the above with the following lemma, which will allow us to apply the ergodicity criterion given in Theorem 19 .

Lemma 29. Let $G \subset \Pi_{d}$ be a subgroup which acts transitively on $\{1, \ldots, d\}$ for some $d \geq 2$. For each finite subset $J \subset \mathbb{Z}$ and for each $\eta>0$, there is a constant $c=c(\eta, J)>0$ so that for each $h \in \operatorname{Hom}(\Gamma, G)$ that satisfies the condition that the subgroup generated by $\left\{h\left(\gamma_{j}\right): j \in J\right\}$ acts transitively on $\{1, \ldots, d\}$, there is a connected subsurface $\tilde{U}_{h}$ of the cover $\left(\tilde{S}_{h}, \tilde{\alpha}_{h}\right)$ associated to $h$, so that Area $\left(\tilde{S}_{h} \backslash \tilde{U}_{h}\right)<\eta \operatorname{Area}\left(\tilde{S}_{h}\right)$ and

$$
\epsilon(t)^{4} \mathcal{D}_{t}^{-2}>c \quad \text { for each } t \in \mathbb{R} \text { with }|t| \leq \frac{\ln (2)}{2},
$$

where $\epsilon(t)$ represents a lower bound for the distance from points in $\tilde{U}_{t}$ to points in $\Sigma$ and $\mathcal{D}_{t}$ is an upper bound for the diameter of $\tilde{U}_{h}$ both measured using the pullback metric dist $t_{t}$ as in Theorem 19.

We note that the expression $\epsilon(t)^{4} \mathcal{D}_{t}^{-2}$ is the function inside the integral in Theorem 19 in the special case of considering a single connected subsurface (i.e., $C_{t} \equiv 1$ ).

Proof. We will explicitly describe how to build $\tilde{U}_{h} \subset\left(\tilde{S}_{h}, \tilde{\alpha}_{h}\right)$. We will define $U$ to be a subsurface of $\left(S_{2}, \alpha_{2}\right)$, which only depends on $J$ and $\eta$. Recall that $\left(S_{2}, \alpha_{2}\right)$ was built from a single unit area square with edge identifications. To build $U$, start with a smaller concentric square with area equal to $1-\eta$. Then for each $j \in J$, consider the edge of the surface labeled $j$. Attach to the concentric square a "handle" passing through the edge, which stays a bounded distance away from $\Sigma$ (the points added in the metric completion). See Figure 3 for an example. Then, we define $\tilde{U}_{h}$ to be the preimage of $U$ under the covering map $\widehat{S}_{h} \rightarrow S$. We note that $\tilde{U}_{h}$ is connected because of our condition that $\left\{h\left(\gamma_{j}\right): j \in J\right\}$ acts transitively.

The dist $t_{t}$ distances $\epsilon(t)$ and $\mathcal{D}_{t}$ are the same as the corresponding distances measured using $g^{t}(\tilde{U})$ in $g^{t}\left(\tilde{S}_{h}, \tilde{\alpha}_{h}\right)$. Note that the minimal distance from the boundary of $g^{t}\left(\tilde{U}_{h}\right)$ to the metric completion in $g^{t}\left(\tilde{S}_{h}, \tilde{\alpha}_{h}\right)$ is the same as the minimal distance from $g^{t}(U)$ to the metric completion in $g^{t}\left(S_{2}, \alpha_{2}\right)$. This distance is always positive and varies continuously in $t$, so we can get a uniform lower bound which holds when $|t| \leq \frac{1}{2} \ln 2$. Similarly, the diameter of $g^{t}\left(\tilde{U}_{h}\right)$ varies continuously in $t$, so we can get an upper bound on the diameter which is uniform in $t$ with $|t| \leq \frac{1}{2} \ln 2$. Finally observe that up to translation equivalence there are 
only finitely many $\tilde{U}_{h}$ as we vary $h$. (The geometry of $\tilde{U}_{h}$ only depends on the restriction of $h$ to $J$.) Thus, we can get an upper bound on the diameter of which is uniform in both $h$ satisfying our condition and $t$ satisfying $|t| \leq \frac{1}{2} \ln 2$.

We need a mechanism to ensure that $h$ satisfies the condition of the lemma (i.e., for some fixed $J \subset \mathbb{Z}$ the subgroup generated by $\left\{h\left(\gamma_{j}\right): j \in J\right\}$ acts transitively on $\left.\{1, \ldots, d\}\right)$, given conditions on the $G$-sequence $\mathfrak{g}(h)$. This mechanism is a corollary of Lemma 27 .

Corollary 30. Suppose $h$ has $G$-sequence $\mathbf{g}=\mathfrak{g}(h)$. Let $m, k \in \mathbb{Z}$ with $m>0$. Then, if the subgroup generated by $\left\{\mathbf{g}_{j}: k-m \leq j \leq k+m\right\}$ acts transitively on $\{1, \ldots, d\}$, then so does the subgroup generated by $\left\{h \circ \phi_{\beta}^{-k}\left(\gamma_{j}\right):-m+1 \leq j \leq m+1\right\}$.

Proof. Using Lemma 27, we can find an expression for each $\mathbf{g}_{j}$ with $k-m \leq j \leq k+m$ in terms of a $\left\{h \circ \phi_{\beta}^{-k}\left(\gamma_{j}\right):-m+1 \leq j \leq m+1\right\}$.

Proof of Theorem 23. We will exhibit an ergodic cover $\left(\tilde{S}_{h}, \tilde{\alpha}_{h}\right)$ by verifying the conditions of Theorem 19. As stated, this theorem involves verifying that for each $\eta>0$, a certain integral is infinite. However, it really only involves proving it for arbitrarily small $\eta$, since if the statement is true for $\eta>0$ then it is also true for any $\eta^{\prime}>\eta$ with the same subsurfaces and geometric functions chosen. Hence, it suffices to verify that this integral is infinite for each of $\eta_{i}>0$ in a sequence tending to zero.

The main tool to get infinite integrals is Lemma 29. We will only apply this Lemma to sets $J$ of the form

$$
J_{m}=\{n \in \mathbb{Z}:|n| \leq m\} .
$$

Let $\operatorname{Hom}_{m} \subset \operatorname{Hom}(\Gamma, G)$ be the collection of all $h$ so that the subgroup generated by $\left\{h\left(\gamma_{n}\right): n \in J_{m}\right\}$ acts transitively on $\{1, \ldots, d\}$. Then the conditions needed for applying Lemma 29 with $J=J_{m}$ can be succinctly stated as $h \in \operatorname{Hom}_{m}$. Let $c_{i, m}=c\left(\eta_{i}, J_{m}\right)$ be the constants given by Lemma 29. Observe that $D(\phi)=g^{\ln 2}$. This means that

$$
g^{k \ln 2}\left(\tilde{S}_{h}, \tilde{\alpha}_{h}\right)=\left(\tilde{S}_{h \circ \phi^{-k}}, \tilde{\alpha}_{h \circ \phi^{-k}}\right) .
$$

So, assuming that $h \circ \phi^{-k} \in \mathrm{Hom}_{m}$, the contribution to the integral in Theorem 19 taken with $\eta=\eta_{i}$ and $t \in\left[\left(k-\frac{1}{2}\right) \ln 2,\left(k+\frac{1}{2}\right) \ln 2\right]$ is at least $c_{i, m} \ln 2$.

We need the total contribution to the integrals for each $\eta_{i}$ to be infinite. For each $i$, choose a sequence $N_{i, m}$ so that

$$
\sum_{m}^{\infty} N_{i, m} c_{i, m} \ln 2=+\infty .
$$

(The number $N_{i, m}$ representing a number of times we contribute $c_{i, m} \ln 2$ to the integral using the method of the previous paragraph.) Now define the sequence

$$
N_{m}=\max \left\{N_{1, m}, N_{2, m}, \ldots, N_{m, m}\right\} \quad \text { and observe } \quad \sum_{m} N_{m} c_{i, m} \ln 2=+\infty \quad \text { for all } i .
$$

Observe that we have the following criterion for ergodicity:

Claim. Fix $h$. If there is a sequence of pairwise disjoint subsets $K_{m} \subset \mathbb{N}$ defined for $m \geq m_{0}$ for some $m_{0}>0$ so that $\left|K_{m}\right| \geq N_{m}$ and $h \circ \phi^{-k} \in \operatorname{Hom}_{m}$ for each $k \in K_{m}$, then the translation flow on $\left(\tilde{S}_{h}, \tilde{\alpha}_{h}\right)$ is ergodic.

The proof of the claim is that by using Lemma 29 with $J=J_{m}$ on $h \circ \phi^{-k}$ at times $t \in\left[\left(k-\frac{1}{2}\right) \ln 2,\left(k+\frac{1}{2}\right) \ln 2\right]$ where $k \in K_{m}$ we get a contribution of $c_{i, m} \ln 2$ to the integral 
associated to $\eta_{i}$. These contributions occur at least $N_{m}$ times when $m \geq m_{0}$, so each of the integrals is infinite by (21).

Now we will explain how to meet the hypotheses of this claim. First we prefer to work with the $G$-sequence $\mathbf{g}=\mathfrak{g}(h)$ than with $h$ directly. By Corollary 30, we can guarantee that $h \circ \phi^{-k} \in \operatorname{Hom}_{m}$ if $\sigma^{k}(\mathbf{g}) \in \operatorname{Gen}(m)$ where $\operatorname{Gen}(m)$ denotes the set of $\mathbf{g}^{\prime} \in G^{\mathbb{Z}}$ so that the subgroup generated by $\left\{\mathbf{g}_{j}^{\prime}:|j|<m\right\}$ acts transitively on $\{1, \ldots, d\}$. Hence we can replace the condition $h \circ \phi^{-k} \in \operatorname{Hom}_{m}$ in our Claim with the condition that $\sigma^{k}(\mathbf{g}) \in \operatorname{Gen}(m)$.

We will now produce a $\mathbf{g}$ together with subsets $K_{m}$ as above so that $\sigma^{k}(\mathbf{g}) \in G e n(m)$ when $k \in K_{m}$. To do this we only need to specify a tail for $\mathbf{g}$; i.e., the values for $\mathbf{g}_{k}$ for $k$ large. Say that a word is an element $w \in G^{l}$ for some $l \in \mathbb{N}$. We use $|w|$ to denote the length of $w$. We will express this tail for $\mathbf{g}$ as an infinite concatenation of words. For $i \in\{1,2\}$ choose words $w_{i}$ so that the group elements appearing in the words generate the subgroup $H_{i}$ as in the statement of the Theorem. Let $e$ denote the identity element of $G$, and let $e^{j}$ denote word formed by repeating the identity $j$ times. Observe that if $\ell+\left|w_{1}\right|+\left|w_{2}\right|=2 m-1$ and the word

$$
\mathbf{g}_{-m+1}^{\prime} \mathbf{g}_{-m+2}^{\prime} \ldots \mathbf{g}_{m-1}^{\prime} \quad \text { equals } w_{1} e^{\ell} w_{2} \text { or } w_{2} e^{\ell} w_{1}
$$

then $\mathbf{g}^{\prime} \in G e n(m)$ since by hypothesis the group generated by $H_{1} \cup H_{2}$ acts transitively on $\{1, \ldots, d\}$. Let $m_{0}$ be such that $2 m_{0}-1 \geq 1+\left|w_{1}\right|+\left|w_{2}\right|$. Let $\left(m_{j}\right)$ be any sequence in the set $\left\{m \in \mathbb{N}: m \geq m_{0}\right\}$ so that each $m \geq m_{0}$ appears $N_{m}$ times in the sequence. For each $j$ define $\ell_{j}$ so that $\ell_{j}+\left|w_{1}\right|+\left|w_{2}\right|=2 m_{j}-1$. Now assume $\mathbf{g}$ has a tail of the form

$$
w_{1} e^{\ell_{1}} w_{2} e^{\ell_{2}} w_{1} e^{\ell_{3}} w_{2} e^{\ell_{4}} w_{1} \ldots
$$

Then for each $j$ there is a $k_{j}$ so that when $\mathbf{g}^{\prime}=\sigma^{k_{j}}(\mathbf{g})$ the word of 22 taken with $m=m_{j}$ has the form of either $w_{1} e^{\ell_{j}} w_{2}$ or $w_{2} e^{\ell_{j}} w_{1}$ depending on the parity of $j$. In particular, $\sigma^{k_{j}}(\mathbf{g}) \in \operatorname{Gen}\left(m_{j}\right)$ for each $j$. By construction, $m$ appears $N_{m}$ times in the sequence $\left(m_{j}\right)$, so by application of the Claim we see that if $\mathfrak{g}(h)=\mathbf{g}$ then the translation flow on $\left(\tilde{S}_{h}, \tilde{\alpha}_{h}\right)$ is ergodic.

Recall that we can improve ergodicity to unique ergodicity using Corollary 3 since the 2 -adic odometer is uniquely ergodic.

It remains to explain that the orbit of $\left(\tilde{S}_{h}, \tilde{\alpha}_{h}\right)$ under $\phi$ only accumulates on disconnected covers as stated in the Theorem. Observe that the $\mathbf{g}$ we constructed is $p$-ready, where $p:\{n \in \mathbb{Z}: n \geq a\} \rightarrow\{0,1,2\}$ is defined so that $a$ represents the position of the first letter in the tail (23), and $p(a+n)$ is determined by the $n+1$-st position in this tail: If the $n+1$-st position belongs to an $e^{\ell_{*}}$ word we define $p(a+n)=0$ and if it belongs to a $w_{i}$ word we define $p(a+n)=i$. Since each $m$ appears only finitely many times in the sequence $m_{j}$, we see that the length of the intervals $I(p, n)$ where $p(n)=0$ tends to infinity. Thus as an application of Proposition 28, $\left(\tilde{S}_{h}, \tilde{\alpha}_{h}\right)$ only accumulates on disconnected covers as desired.

7.2. The ladder surface. For this subsection, we let $\left(S_{L}, \alpha_{L}\right)$ denote the infinite genus translation surface of Figure 4, which we call the ladder surface. It can be constructed from a region in the plane bounded by countably many horizontal and vertical edges. We have labeled the edges using the set $\mathbb{Z}^{*}=\mathbb{Z} \backslash\{0\}$. Edges with the same label are glued by translation to form the surface, which does not include endpoints of these edges or the limiting point in the top right of the region. Let $\varphi$ denote the golden ratio, $\frac{\sqrt{5}+1}{2}$. An edge with label $j \in \mathbb{Z}^{*}$ has length given by $\varphi^{-|j|}$. This information specifies the geometry of the surface. We have also selected a basepoint $s_{0}$ for our surface in the figure. 

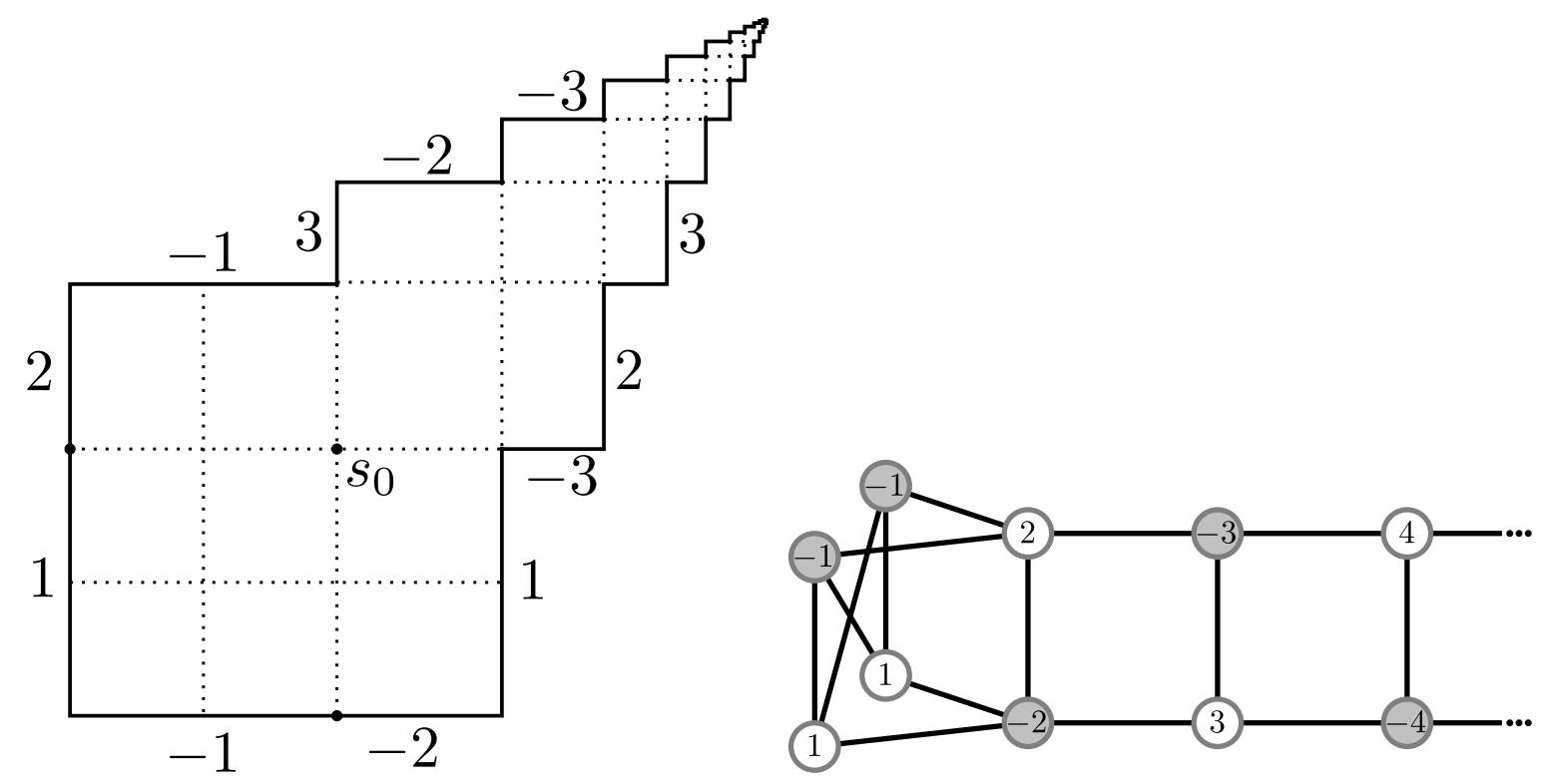

Figure 4. Left: The ladder surface $\left(S_{L}, \alpha_{L}\right)$. Right: The graph used in Thurston's construction to build the ladder surface.

The surface is built using Thurston's construction [Thu88, §6] from the graph $\mathcal{G}$ depicted in Figure 4. We will briefly review this construction. The surface $\left(S_{L}, \alpha_{L}\right)$ has horizontal and vertical cylinder decompositions with cylinders bounded by dotted lines in Figure 4 . From these cylinders one can build a bipartite graph: the white vertices consist of the horizontal cylinders, the gray vertices consist of the vertical cylinders and an edge is drawn between two vertices for each intersection between the corresponding two cylinders. The vertices of $\mathcal{G}$ have been labeled in the figure to match the label of the edge passed through by the corresponding cylinder. (Two adjacent cylinders pass through edges labeled -1 and 1.) For such a connected graph, for any $A>0$ there is at most one way to assign widths to the cylinders so that the area of the resulting surface is $A$ and the moduli (ratio of widths to circumferences) of all these cylinders are equal: this assignment viewed as a real valued function defined on the vertices of the graph must be an eigenfunction for the adjacency operator. (This eigenfunction always exists for finite graphs by the PerronFrobenius theorem, while uniqueness is guaranteed in the infinite case by an analogous result [VJ68, Theorem 2.2].) This forces the lengths of edges of the region defining our surface to be as mentioned above (up to uniform scaling).

Once the moduli of the cylinders are all equal, an observation of Thurston guarantees existence of two affine automorphisms of $\left(S_{L}, \alpha_{L}\right)$ with parabolic derivative and horizontal and vertical eigenvectors respectively preserving the horizontal and vertical cylinders in the decompositions. In the case of our surface, there is an additional reflective symmetry in a line of slope 1 . To generate these three affine automorphisms it is sufficient to use $\psi$ which performs a single left Dehn twist in each of the countably many vertical cylinders, and the reflective symmetry $\rho$. The derivatives are given by

$$
D(\psi)=\left[\begin{array}{rr}
1 & 0 \\
2 \varphi & 1
\end{array}\right] \quad \text { and } \quad D(\rho)=\left[\begin{array}{ll}
0 & 1 \\
1 & 0
\end{array}\right]
$$


The composition $\rho \circ \psi \circ \rho$ performs a right Dehn twist in each of the horizontal cylinders. All these affine automorphisms fix the basepoint $s_{0}$ depicted in Figure 4 . We define Aff' to be the subgroup of $\operatorname{Aff}\left(S_{L}, \alpha_{L}\right)$ generated by $\psi$ and $\rho$. The group Aff ${ }^{\prime}$ is isomorphic to the free product $\mathbb{Z} *(\mathbb{Z} / 2 \mathbb{Z})$ and contains no translation automorphisms.

Remark 31. It is unknown to the authors if Aff $^{\prime}=\operatorname{Aff}\left(S_{L}, \alpha_{L}\right)$.

Recall that $\mathcal{O}(S, \alpha)$ is naturally identified with $S L_{ \pm}(2, \mathbb{R}) / V(S, \alpha)$. See the discussion below (1). Let $V^{\prime}=D\left(A f f^{\prime}\right)$. Since $A f f^{\prime}$ contains no translation automorphisms, the derivative map $D: A f f^{\prime} \rightarrow V^{\prime}$ is a group isomorphism. Unfortunately we do not know if $V^{\prime}=V(S, \alpha)$, so all we get is a covering map:

$$
S L_{ \pm}(2, \mathbb{R}) / V^{\prime} \rightarrow \mathcal{O}(S, \alpha)
$$

Fortunately, if a geodesic recurs in $S L_{ \pm}(2, \mathbb{R}) / V^{\prime}$, then its image in $\mathcal{O}(S, \alpha)$ also recurs. So non-divergence of $g^{t} A V^{\prime}$ implies ergodicity of the translation flow on $A(S, \alpha)$ by Corollary 20. We will be working throughout this section without knowing precise information about the Veech group and this will create difficulties which will be dealt with. It turns out we will be able to get by only knowing the following:

Proposition 32. The Veech group $V\left(S_{L}, \alpha_{L}\right)$ is discrete.

Proof. Consider the maximal vertical cylinder $C_{-2}$ in $S_{L}$ which passes through the edge labeled -2 in Figure 4. We chose this because it has the longest circumference of all vertical cylinders. Suppose $A \in S L(2, \mathbb{R})$ is close enough to the identity so that a closed vertical segment of length equal to the circumference of $C_{-2}$ immerses inside of $A\left(C_{-2}\right)$. Now suppose that $A \in V\left(S_{L}, \alpha_{L}\right)$. Then there is a translation isomorphism $e: A\left(S_{L}, \alpha_{L}\right) \rightarrow\left(S_{L}, \alpha_{L}\right)$. The restriction $\left.e\right|_{\ell}$ must not given an embedding of $\ell$ because all vertical trajectories on $\left(S_{L}, \alpha_{L}\right)$ close up at or before this length. Since $\left.e\right|_{C_{-2}}$ is an embedding we see that $\ell$ must close up on $A\left(C_{-2}\right)$. Thus $A\left(C_{-2}\right)$ must be a vertical cylinder. Because $C_{-2}$ has singularities in its boundary so must $A\left(C_{-2}\right)$, so that $A\left(C_{-2}\right)$ is a maximal cylinder. Area considerations show that $A\left(C_{-2}\right)=C_{-2}$. We conclude that $A$ has a vertical eigenvector with eigenvalue 1 . A symmetric argument implies that if $A$ is taken from a small enough open neighborhood of the identity then $A$ has a horizontal eigenvector with eigenvalue 1. By choosing our neighborhood small enough, both arguments hold and we see that the only element of $V\left(S_{L}, \alpha_{L}\right)$ in this neighborhood is the identity.

We will need to understand $S L_{ \pm}(2, \mathbb{R}) / V^{\prime}$ for later arguments. The group $S L_{ \pm}(2, \mathbb{R})$ can be identified with the flag bundle of the hyperbolic plane (where a flag is a choice of a unit tangent vector and an orthogonal unit tangent vector taken from the same tangent plane). Here the hyperbolic plane is $O(2) \backslash S L_{ \pm}(2, \mathbb{R})$. The group $S L_{ \pm}(2, \mathbb{R})$ then acts on the right by isometry. The unit speed geodesics have the form $t \mapsto O(2) g^{t} A$ for some $A \in S L_{ \pm}(2, \mathbb{R})$. The boundary of the hyperbolic plane can be identified with $\mathbb{R P}^{1}=\mathbb{R}^{2} /(\mathbb{R} \backslash\{0\})$. The geodesic $t \mapsto O(2) g^{t} A$ converges as $t \rightarrow+\infty$ to the boundary point representing the vectors which are contracted to zero as $t \rightarrow \infty$, the projective equivalence class of

$$
\left(\begin{array}{l}
x \\
y
\end{array}\right)=A^{-1}\left(\begin{array}{l}
1 \\
0
\end{array}\right) .
$$

We identify the hyperbolic plane $O(2) \backslash S L_{ \pm}(2, \mathbb{R})$ with the upper half plane in such a way so that the projective class of $(x, y)$ viewed as a boundary point is identified with the slope $y / x \in \mathbb{R} \cup\{\infty\}$. 


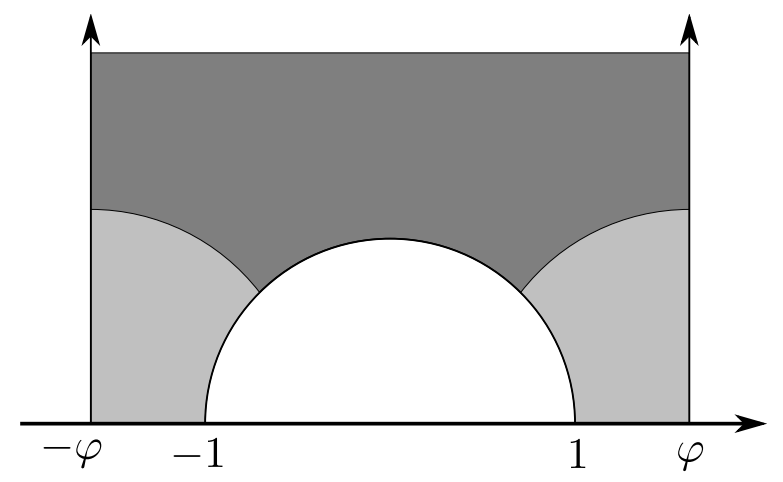

FiguRE 5. A fundamental domain for the action of $V^{\prime}$ on the upper half plane is shown in gray (it extends vertically to $\infty$ ). The geodesic between -1 and 1 is preserved by the reflection $\rho$, and $\psi$ acts by translation by $2 \varphi$. The convex core is in dark gray and extends upward.

We let $V^{\prime}$ denote the group of derivatives of $A f f^{\prime}$. The group $V^{\prime}$ acts discretely on the hyperbolic plane, and Figure 5 shows a fundamental domain for the action. A geodesic ray $t \mapsto O(2) g^{t} A V^{\prime}$ in $S L_{ \pm}(2, \mathbb{R}) / V^{\prime}$ for $t>0$ is non-divergent if and only if the endpoint of the geodesic in the hyperbolic plane lies in the horospherical limit set, which in this case is the limit set of $V^{\prime}$ with fixed points of parabolics removed [BM74, Theorem 2]. The limit set in this case is a Cantor set of Hausdorff dimension larger than $\frac{1}{2}$ [Hoo15, Remark 4.1]. The convex core is the convex hull of the limit set in the hyperbolic plane projected into the surface $O(2) \backslash S L_{ \pm}(2, \mathbb{R}) / V^{\prime}$. A geodesic ray in this surface is non-divergent if and only if it has an $\omega$-limit point in the convex core. The convex core of this surface is depicted in Figure 5 .

Remark 33 (Boundary of convex core). The geodesic in the surface $O(2) \backslash S L_{ \pm}(2, \mathbb{R}) / V^{\prime}$ with monodromy given by the derivative of the commutator $D([\rho, \psi])$ is the boundary of the convex core of this surface.

Theorem 34. Let $A \in S L_{ \pm}(2, \mathbb{R})$, and $\left(S_{L}, \alpha_{L}\right)$ be the ladder surface. Consider the geodesic ray $t \mapsto O(2) g^{t} A V^{\prime}$ in the surface $O(2) \backslash S L_{ \pm}(2, \mathbb{R}) / V^{\prime}$.

- If the ray has an $\omega$-limit point (which must be in the convex core) then the translation flow on $A\left(S_{L}, \alpha_{L}\right)$ is defined for all time almost everywhere and is ergodic.

- If the ray has an $\omega$-limit point in the interior of the convex core then the translation flow on $A\left(S_{L}, \alpha_{L}\right)$ is uniquely ergodic.

Proof. To see the first statement observe that if the geodesic has an $\omega$-limit point then so does the trajectory $t \mapsto g^{t} A\left(S_{L}, \alpha_{L}\right)$ in $\mathcal{O}\left(S_{L}, \alpha_{L}\right)$ because of the covering map (24). The conclusion is then given by Corollary 20.

The second statement is an application of Theorem H.5 of [Hoo15]. To use the theorem we need to verify several hypotheses. First $\left(S_{L}, \alpha_{L}\right)$ arises from Thurston's construction using a graph with no vertices of valence one as with the graph here; see Figure 4. Second in the language of that article we need the trajectory $g^{t} A$ not be asymptotic to an endpoint of a maximal interval of the compliment of the limit set of the subgroup of $V\left(S_{L}, \alpha_{L}\right)$ generated by the horizontal and vertical parabolics. The subgroup generated by these parabolics is the same as $V^{\prime}$ up to finite index, so the two limit sets are identical. This condition that the trajectory is not asymptotic to an endpoint of a complimentary interval is equivalent to 
$O(2) g^{t} A V^{\prime}$ not being asymptotic to the boundary of the convex core in $O(2) \backslash S L_{ \pm}(2, \mathbb{R}) / V^{\prime}$. It is impossible for a geodesic ray with an $\omega$-limit point in the interior of the convex core to be asymptotic to the convex core boundary.

We will be considering double covers of the ladder surface. For $i \in \mathbb{Z}^{*}$, we let $\gamma_{i}$ be one of the two homotopy class of loops which start at the basepoint, cross only the edge labeled $i$, and return to the basepoint. These two homotopy classes are inverses in $\Gamma=\pi_{1}\left(S, s_{0}\right)$. We make the choice of $\gamma_{i}$ so $\gamma_{i}$ moves rightward across the vertical edge labeled $i$ if $i>0$, and moves upward over the horizontal edge labeled $i$ if $i<0$. These curves freely generate the fundamental group, which we denote by $\Gamma=\pi_{1}\left(S, s_{0}\right)$. (As with the the Chamanara surface in the previous section, the curves $\gamma_{i}$ can be chosen so that $S_{L}$ retracts onto the union of the curves which is homeomorphic to a countable bouquet of circles.)

Let $\mathbb{Z}_{2}=\mathbb{Z} / 2 \mathbb{Z}$. Observe that $\mathbb{Z}_{2}$ acts trivially on $\operatorname{Hom}\left(\Gamma, \mathbb{Z}_{2}\right)$ by conjugation so that $\operatorname{TopCov}_{\mathbb{Z}_{2}}\left(S_{L}\right)=\operatorname{Hom}\left(\Gamma, \mathbb{Z}_{2}\right)$; see (6). It can be seen from Remark 9 that $\operatorname{Cov}_{\mathbb{Z}_{2}}\left(S_{L}, \alpha_{L}\right)=$ $\operatorname{TopCov}_{\mathbb{Z}_{2}}\left(S_{L}\right)$, i.e., (7) is a bijection in this case. So in summary,

$$
\operatorname{Cov}_{\mathbb{Z}_{2}}\left(S_{L}, \alpha_{L}\right)=\operatorname{Hom}\left(\Gamma, \mathbb{Z}_{2}\right) .
$$

Since our basepoint is fixed by the affine automorphisms in $A f f^{\prime}$, we have a canonical action of each element in $A f f^{\prime}$ on the space of covers. For example, if $h \in \operatorname{Hom}\left(\Gamma, \mathbb{Z}_{2}\right)$, then $\psi_{*}(h)=h \circ \psi^{-1}$ and $\rho_{*}(h)=h \circ \rho^{-1}$. We will work out this action below.

Notation 35. Let $h \in \operatorname{Hom}\left(\Gamma, \mathbb{Z}_{2}\right)$. We will write $h(i)$ to abbreviate $h\left(\gamma_{i}\right)$ for $i \in \mathbb{Z}^{*}$. (We have a bijection between elements of $\operatorname{Hom}\left(\Gamma, \mathbb{Z}_{2}\right)$ and the set of all functions $\mathbb{Z}^{*} \rightarrow \mathbb{Z}_{2}$.)

It can be observed that the generators of $A f f^{\prime}$ act on $\operatorname{Hom}\left(\Gamma, \mathbb{Z}_{2}\right)$ as follows:

$$
\begin{gathered}
\left(\psi_{*}(h)\right)(i)= \begin{cases}h(i) & \text { if } i<0, \\
h(i)+h(-2) & \text { if } i=1 \\
h(i)+h(-2)+h(-3) & \text { if } i=2, \\
h(i)+h(-i-1)+h(-i)+h(-i+1) & \text { if } i>2 .\end{cases} \\
\qquad\left(\rho_{*}(h)\right)(i)=h(-i) .
\end{gathered}
$$

A key observation is the following:

Proposition 36. The square $\psi_{*}^{2}$ acts trivially on $\operatorname{Hom}\left(\Gamma, \mathbb{Z}_{2}\right)$.

Proof. The action of $\psi_{*}^{2}$ preserves the value of $h(i)$ for $i<0$, and adds a sum of values of $h$ evaluated at negative integers to the values of $h(i)$ for $i>0$. Since adding the same number twice is the same as adding zero in $\mathbb{Z}_{2}, \psi_{*}^{2}$ acts trivially.

We have the following trivial consequence:

Corollary 37. The subgroup of $V^{\prime}$ given by

$$
\tilde{V}^{\prime}=\left\langle R D\left(\psi^{2}\right) R^{-1} \mid R \in V^{\prime}\right\rangle
$$

is a subgroup of the Veech group of any $\left(\tilde{S}_{h}, \tilde{\alpha}_{h}\right) \in \operatorname{Cov}_{\mathbb{Z}_{2}}\left(S_{L}, \alpha_{L}\right)$.

From this we get a covering map (similar to (24)):

$$
S L_{ \pm}(2, \mathbb{R}) / \tilde{V}^{\prime} \rightarrow \mathcal{O}\left(\tilde{S}_{h}, \tilde{\alpha}_{h}\right) .
$$

Observe that a non-divergent geodesic in $S L_{ \pm}(2, \mathbb{R}) / \tilde{V}^{\prime}$ will descend to a non-divergent geodesic in $\mathcal{O}\left(\tilde{S}_{h}, \tilde{\alpha}_{h}\right)$. We have recovered statement (L1) of the introduction: 
Proposition 38. Suppose that $g^{t} A \tilde{V}^{\prime}$ is non-divergent in $S L_{ \pm}(2, \mathbb{R}) / \tilde{V}^{\prime}$. Then for any connected $\left(\tilde{S}_{h}, \tilde{\alpha}_{h}\right) \in \operatorname{Cov}_{\mathbb{Z}_{2}}\left(S_{L}, \alpha_{L}\right)$, the double cover $A\left(\tilde{S}_{h}, \tilde{\alpha}_{h}\right)$ of $A\left(S_{L}, \alpha_{L}\right)$ is not devious and every $A\left(\tilde{S}_{h}, \tilde{\alpha}_{h}\right)$ has uniquely ergodic translation flow.

Proof. Fix $A$ satisfying the first statement and any cover $\left(\tilde{S}_{h}, \tilde{\alpha}_{h}\right)$. From the covering map (29), we know $g^{t} A\left(\tilde{S}_{h}, \tilde{\alpha}_{h}\right)$ is non-divergent in $\mathcal{O}\left(\tilde{S}_{h}, \tilde{\alpha}_{h}\right)$. Thus this cover is not devious and ergodicity follows from Corollary 20. To prove unique ergodicity, it suffices in light of Corollary 3 to show that the translation flow on the surface $A\left(S_{L}, \alpha_{L}\right)$ is uniquely ergodic. This holds unless $O(2) g^{t} A V^{\prime}$ is forward asymptotic to the convex core boundary of $O(2) \backslash S L_{ \pm}(2, \mathbb{R}) / V^{\prime}$ by Theorem 34 . But this convex core boundary lifts to a pair of divergent geodesics in $O(2) \backslash S L_{ \pm}(2, \mathbb{R}) / \tilde{V}^{\prime}$. (This will be justified more below; the wavy lines between light and dark gray regions in Figure 6 denote the lifted boundary of the convex core.)

Our main goal of the section is to find some devious covers, so from the above proposition we will need to consider elements $A \in S L_{ \pm}(2, \mathbb{R})$ so that $g^{t} A \tilde{V}^{\prime}$ is divergent in $S L_{ \pm}(2, \mathbb{R}) / \tilde{V}^{\prime}$. We will show that the existence of such covers is related to a combinatorial rate of divergence of this trajectory. To measure this we need to further develop our understanding of $\tilde{V}^{\prime}$ and the surface $O(2) \backslash S L_{ \pm}(2, \mathbb{R}) / \tilde{V}^{\prime}$.

From (28), the group $\tilde{V}^{\prime}$ is evidently normal in $V^{\prime}$. Let $\Delta=V^{\prime} / \tilde{V}^{\prime}$ be the quotient. This group has a right action as the Deck group of the covering maps

$$
S L_{ \pm}(2, \mathbb{R}) / \tilde{V}^{\prime} \rightarrow S L_{ \pm}(2, \mathbb{R}) / V^{\prime} \quad \text { and } \quad O(2) \backslash S L_{ \pm}(2, \mathbb{R}) / \tilde{V}^{\prime} \rightarrow O(2) \backslash S L_{ \pm}(2, \mathbb{R}) / V^{\prime} .
$$

Since as a group $V^{\prime}=\langle D(\psi), D(\rho)\rangle$ is isomorphic to the free product $\mathbb{Z} * \mathbb{Z}_{2}$, and $\psi^{2}$ is in $\tilde{V}^{\prime}$, the quotient $\Delta$ is isomorphic to the infinite dihedral group which we think of as $\operatorname{Isom}(\mathbb{Z})$, where $\mathbb{Z}$ is given the standard metric. A homomorphism $\delta: V^{\prime} \rightarrow \operatorname{Isom}(\mathbb{Z})$ which factors through $\Delta$ is given by

$$
\delta \circ D(\psi): n \mapsto-n \quad \text { and } \quad \delta \circ D(\rho): n \mapsto 1-n .
$$

The action of the Veech group on $\operatorname{Cov}_{\mathbb{Z}_{2}}\left(S_{L}, \alpha_{L}\right)=\operatorname{Hom}\left(\Gamma, \mathbb{Z}_{2}\right)$ induces an action of $\operatorname{Isom}(\mathbb{Z})$, where

$$
(\delta \circ D(\psi))_{*}=\psi_{*} \quad \text { and } \quad(\delta \circ D(\rho))_{*}=\rho_{*} .
$$

We will now give a cartoon description of the surface $O(2) \backslash S L_{ \pm}(2, \mathbb{R}) / \tilde{V}^{\prime}$. We can deform our fundamental domain for the $V^{\prime}$ action so that it looks like the left side of Figure 6 . The advantage here is that two copies of this domain can be joined together by a Euclidean rotation by $180^{\circ}$ about the point $\infty$. This rotation is then representing the order two action on $S L_{ \pm}(2, \mathbb{R}) / \tilde{V}^{\prime}$ given by the right action of $D(\psi)$. The surface $O(2) \backslash S L_{ \pm}(2, \mathbb{R}) / \tilde{V}^{\prime}$ can be tiled by copies of this fundamental domain as shown on the right side of the figure. We have drawn this in such a way so that the action of the Deck group $\operatorname{Isom}(\mathbb{Z})$ appears natural. In particular, for each $n \in \mathbb{Z}$, we can join together two copies of the fundamental domain together about the point labeled $\infty_{n}$. We call this region $F_{n}$; these regions are depicted in the figure. The isometry $\delta \circ D(\psi): n \mapsto-n$ acts by a rotation by $180^{\circ}$ about the point $\infty_{0}$. The isometry $\delta \circ D(\rho): n \mapsto 1-n$ acts by a Euclidean reflection in the line forming the boundary between $F_{0}$ and $F_{1}$. We observe that $\operatorname{Isom}(\mathbb{Z})$ acts in a natural way on these regions as labeled by $\mathbb{Z}$. Namely, we can think of these regions as subsets of $O(2) \backslash S L_{ \pm}(2, \mathbb{R}) / \tilde{V}^{\prime}$ and

$$
F_{n} R^{-1}=F_{\delta(R)(n)} \quad \text { for all } R \in V^{\prime} \text { and } n \in \mathbb{Z} \text {. }
$$



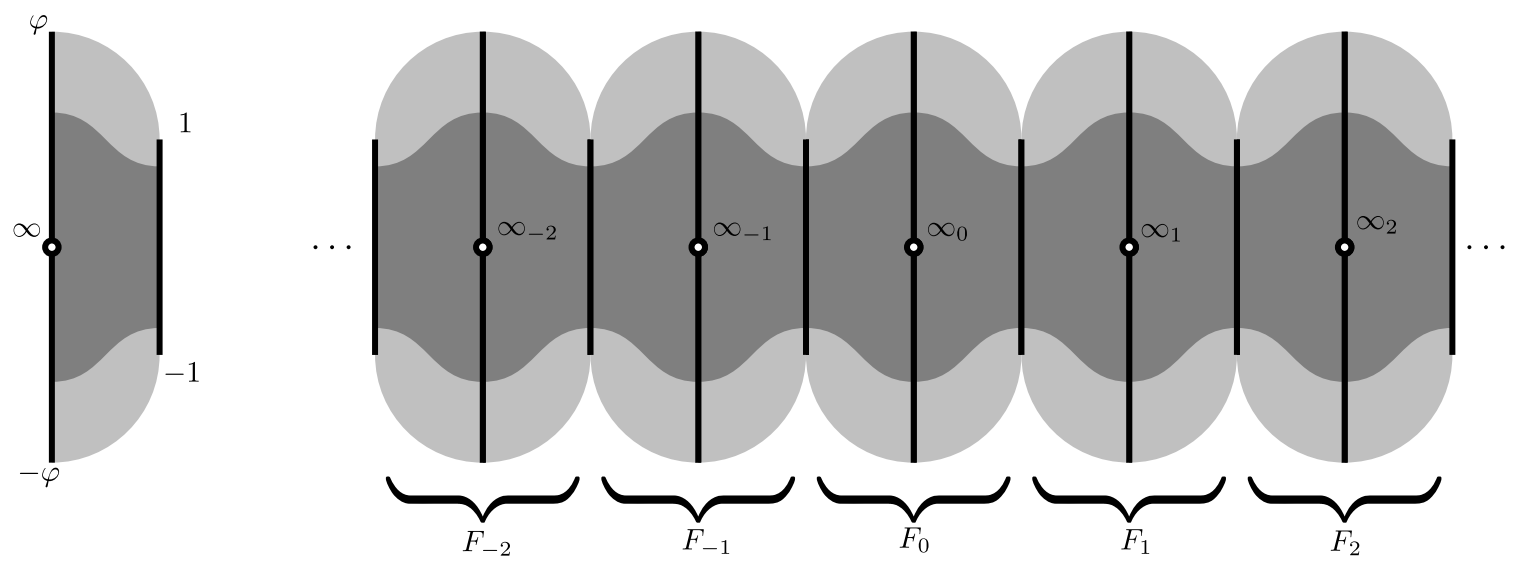

Figure 6. Left: A caricature of the fundamental domain shown in Figure 5. Geodesic boundaries are drawn in black. Right: The quotient surface $O(2) \backslash S L_{ \pm}(2, \mathbb{R}) / \tilde{V}^{\prime}$ tiled by copies of this fundamental domain.

The actual action is somewhat subtle: the translation

$$
\tau^{m}=\delta \circ D\left((\rho \circ \psi)^{m}\right): n \mapsto n+m
$$

acts by translation by $m$ in the figure when $m$ is even, but when $m$ is odd $\tau^{m}$ acts as a glide reflection carrying each $F_{n}$ to $F_{n+m}$.

We use the regions $F_{n}$ in $S L_{ \pm}(2, \mathbb{R}) / \tilde{V}^{\prime}$ to code $g^{t}$-trajectories in $S L_{ \pm}(2, \mathbb{R}) / \tilde{V}^{\prime}$ arising from non-divergent trajectories in $S L_{ \pm}(2, \mathbb{R}) / V^{\prime}$ :

Proposition 39 (Coding walk). Let $A \in S L(2, \mathbb{R})$. If the trajectory $g^{t} A V^{\prime}$ is non-divergent in forward time on $S L_{ \pm}(2, \mathbb{R}) / V^{\prime}$, then there is a sequence of countably many times

$$
0=t_{0}<t_{1}<t_{2}<\ldots
$$

with $\lim _{k \rightarrow \infty} t_{k}=+\infty$ and a sequence of integers $\left\{n_{k}: k=0,1,2, \ldots\right\}$ so that

$$
O(2) g^{t} A \tilde{V}^{\prime} \in F_{n_{k}} \quad \text { whenever } t_{k}<t<t_{k+1} \text {. }
$$

Furthermore, $n_{k+1} \in\left\{n_{k}+1, n_{k}-1\right\}$ for each $k \geq 0$.

In other words, the lift of a non-divergent geodesic on $O(2) \backslash S L_{ \pm}(2, \mathbb{R}) / V^{\prime}$ to a geodesic on $O(2) \backslash S L_{ \pm}(2, \mathbb{R}) / \tilde{V}^{\prime}$ gives rise to a walk on the integers. We'll call this walk the coding walk of the geodesic $g^{t} A \tilde{V}^{\prime}$.

Proof. If this is not true, then the geodesic change regions only finitely many times. Then the geodesic eventually stays in one region, say $F_{n}$. But, we can see that the only geodesics that stay within $F_{n}$ forever are geodesics which exit the cusp $\infty_{n}$ or which exit the preimage of the convex core of $O(2) \backslash S L_{ \pm}(2, \mathbb{R}) / V^{\prime}$ in $F_{n}$. (The region $F_{n}$ is an annulus with parabolic monodromy.) Both possibilities contradict the non-divergence of $g^{t} A V^{\prime}$ in $S L_{ \pm}(2, \mathbb{R}) / V^{\prime}$.

The following is a tool which will be useful to obtain convergence in $\tilde{O}_{\mathbb{Z}_{2}}\left(S_{L}, \alpha_{L}\right)$ using the coding walk.

Lemma 40. Let $U \subset O(2) \backslash S L_{ \pm}(2, \mathbb{R}) / V^{\prime}$ be a cusp neighborhood given by the points in the fundamental domain of Figure 5 with imaginary part larger than 2 . Let $C_{d}$ be a metric $d$-neighborhood of the convex core of $\subset O(2) \backslash S L_{ \pm}(2, \mathbb{R}) / V^{\prime}$. Let $A \in S L(2, \mathbb{R})$ and assume 
$g^{t} A V^{\prime}$ is non-divergent in forward time on $S L_{ \pm}(2, \mathbb{R}) / V^{\prime}$. Let $\left\{n_{k}\right\}$ be the associated coding walk. Let $\left(\tilde{S}_{h}, \tilde{\alpha}_{h}\right) \in \operatorname{Cov}_{\mathbb{Z}_{2}}\left(S_{L}, \alpha_{L}\right)$. There is a compact set $K \subset S L_{ \pm}(2, \mathbb{R})$ so that if $t$ lies in the interval $\left[t_{k}, t_{k+1}\right]$ associated to $n_{k}$ (as in Proposition 39) and $O(2) g^{t} A V^{\prime} \in C_{d} \backslash U$ then there is a $M \in K$ so that

$$
g^{t} A\left(\tilde{S}_{h}, \tilde{\alpha}_{h}\right)=M\left(\tilde{S}_{\tau_{*}^{-n_{k}}(h)}, \tilde{\alpha}_{\tau_{*}^{-n_{k}}(h)}\right) \quad \text { in } \tilde{O}_{\mathbb{Z}_{2}}\left(S_{L}, \alpha_{L}\right) .
$$

Moreover, for any $k$ sufficiently large, there exists such a $t \in\left[t_{k}, t_{k+1}\right]$.

Proof. Recall there is a covering map $O(2) \backslash S L_{ \pm}(2, \mathbb{R}) / \tilde{V}^{\prime} \rightarrow O(2) \backslash S L_{ \pm}(2, \mathbb{R}) / V^{\prime}$. Consider the region $F_{0}$ in the domain. Let $F_{0}^{\prime} \subset O(2) \backslash S L_{ \pm}(2, \mathbb{R}) / \tilde{V}^{\prime}$ be the $d$-neighborhood of preimage of the convex core of $O(2) \backslash S L_{ \pm}(2, \mathbb{R}) / V^{\prime}$ in $F_{0}$ with the preimage of $U$ removed. Then $F_{0}^{\prime}$ is compact and this covering map sends $F_{0}^{\prime}$ onto $C_{d} \backslash U$. Let $\tilde{F}_{0}^{\prime} \subset S L_{ \pm}(2, \mathbb{R}) / \tilde{V}^{\prime}$ be the preimage of $F_{0}^{\prime}$ under projection to $O(2) \backslash S L_{ \pm}(2, \mathbb{R}) / \tilde{V}^{\prime}$. Then $\tilde{F}_{0}^{\prime}$ is also compact and so there is a compact set $K \subset S L_{ \pm}(2, \mathbb{R})$ which projects to $\tilde{F}_{0}^{\prime}$ under the quotient map $S L_{ \pm}(2, \mathbb{R}) \rightarrow S L_{ \pm}(2, \mathbb{R}) / \tilde{V}^{\prime}$

Now consider a $t \in\left[t_{k}, t_{k+1}\right]$ so that $O(2) g^{t} A V^{\prime} \in C_{d} \backslash U$. Then by definition of the coding sequence, $O(2) g^{t} A \tilde{V}^{\prime} \in F_{n_{k}}$. Then

$$
\tau^{-n_{k}}\left(O(2) g^{t} A \tilde{V}^{\prime}\right) \in F_{0}
$$

see (33) and (34). Since $O(2) g^{t} A V^{\prime}$ is asymptotic to the convex core in $O(2) \backslash S L_{ \pm}(2, \mathbb{R}) / V$, for $k$ sufficiently large the point in (35) actually lies in $F_{0}^{\prime}$. Observe also that

$$
\tau^{-n_{k}}\left(O(2) g^{t} A \tilde{V}^{\prime}\right)=O(2) g^{t} A D\left((\rho \circ \psi)^{n_{k}}\right) \tilde{V}^{\prime},
$$

so that from the above there is an $R \in \tilde{V}^{\prime}$ and an $M \in K$ so that

$$
g^{t} A D\left((\rho \circ \psi)^{n_{k}}\right) R=M \text {. }
$$

Now observe that

$$
g^{t} A\left(\tilde{S}_{h}, \tilde{\alpha}_{h}\right)=M \cdot\left(R^{-1} \circ D\left((\rho \circ \psi)^{-n_{k}}\right)\left(\tilde{S}_{h}, \tilde{\alpha}_{h}\right) \quad \text { in } \tilde{O}_{\mathbb{Z}_{2}}\left(S_{L}, \alpha_{L}\right),\right.
$$

where we think of the expression to the right of $M$. as the action of the Veech group of $\left(S_{L}, \alpha_{L}\right)$ on $\operatorname{Cov}_{\mathbb{Z}_{2}}\left(S_{L}, \alpha_{L}\right)$. Here $\operatorname{Cov}_{\mathbb{Z}_{2}}\left(S_{L}, \alpha_{L}\right)=\operatorname{Hom}\left(\Gamma, \mathbb{Z}_{2}\right)$; see (25). Thus, the action of $V^{\prime}$ on $\operatorname{Hom}\left(\Gamma, \mathbb{Z}_{2}\right)$ factors through $\Delta=V^{\prime} / \tilde{V}^{\prime}$ and in particular,

$$
\left(R^{-1} \circ D\left((\rho \circ \psi)^{-n_{k}}\right)\left(\tilde{S}_{h}, \tilde{\alpha}_{h}\right)=\left(\tilde{S}_{\tau_{*}^{-n_{k}}(h)}, \tilde{\alpha}_{\tau_{*}^{-n_{k}}(h)}\right) \quad \text { in } \operatorname{Cov}_{\mathbb{Z}_{2}}\left(S_{L}, \alpha_{L}\right) .\right.
$$

Finally, to obtain such a $t \in\left[t_{k}, t_{k+1}\right]$ for $k$ sufficiently large, observe that because $g^{t} A V^{\prime}$ is asymptotic to the convex core in $S L_{ \pm}(2, \mathbb{R}) / V^{\prime}$, it eventually lies in $C_{d}$. Values of $t=t_{k}$ and $t=t_{k+1}$ satisfy the statement because at these times $O(2) g^{t_{k}} A \tilde{V}^{\prime}$ lies in the boundary of a region, so the corresponding point $O(2) g^{t_{k}} A V^{\prime}$ in $O(2) \backslash S L_{ \pm}(2, \mathbb{R}) / V^{\prime}$ lies on the geodesic joining -1 to 1 in Figure 5 and in particular this point does not lie in $U$.

We already understand the ergodic properties of the translation flow of covers when $g^{t} A \tilde{V}^{\prime}$ is non-divergent; see Proposition 38 . This non-divergence can be captured by the coding walk:

Proposition 41. Assume $g^{t} A V^{\prime}$ is non-divergent in forward time on $S L_{ \pm}(2, \mathbb{R}) / V^{\prime}$. The trajectory $g^{t} A \tilde{V}^{\prime}$ is non-divergent in $S L_{ \pm}(2, \mathbb{R}) / \tilde{V}^{\prime}$ if and only if the coding walk $\left\langle n_{k}\right\rangle$ is recurrent, i.e., there is an $n \in \mathbb{Z}$ so that $n_{k}=n$ for infinitely many $k$. 
Proof. First suppose $g^{t} A \tilde{V}^{\prime}$ is non-divergent. Then $g^{t} A \tilde{V}^{\prime}$ has an $\omega$-limit point in $S L_{ \pm}(2, \mathbb{R}) / \tilde{V}^{\prime}$. This limit point must lie in some region $F_{n}$. Proposition 39 tells us we must visit $F_{n}$ infinitely often, so infinitely many $n_{k}=n$. Now suppose the coding walk recurs to $n$. Then infinitely many times the trajectory $g^{t} A \tilde{V}^{\prime}$ moves from $F_{n-1}$ to $F_{n}$ or from $F_{n+1}$ to $F_{n}$. This means that the trajectory $g^{t} A \tilde{V}^{\prime}$ infinitely often visits a compact neighborhood of the common boundary between two adjacent regions. But then the trajectory must have an $\omega$-limit point in this compact neighborhood, and so the trajectory is non-divergent.

The proposition above tells us that the only way devious covers could exist is if the coding walk does not recur. In this case $\lim n_{k}=+\infty$ or $\lim n_{k}=-\infty$. Theorem 1 tells us that covers which are not devious have ergodic translation flow. So, it remains to understand the devious covers when $\lim n_{k}= \pm \infty$. We now state our main result of the section.

Theorem 42 (Main result on double covers of the ladder surface). Let $A \in S L(2, \mathbb{R})$ and suppose that the geodesic $g^{t} A V^{\prime}$ is non-divergent in $S L_{ \pm}(2, \mathbb{R}) / V^{\prime}$. Let $\left\langle n_{k}\right\rangle$ be the coding walk of the geodesic $g^{t} A \tilde{V}^{\prime}$, and assume this walk does not recur. Then $\lim _{k \rightarrow \infty} n_{k}=s \infty$ where $s \in\{ \pm 1\}$ is a sign. In this case, there exist covers $\left(\tilde{S}_{h}, \tilde{\alpha}_{h}\right) \in \operatorname{Cov}_{\mathbb{Z}_{2}}\left(S_{L}, \alpha_{L}\right)$ so that $A\left(\tilde{S}_{h}, \tilde{\alpha}_{h}\right)$ is a devious double cover of $A\left(S_{L}, \alpha_{L}\right)$. We define the growth exponent of the visit count to be

$$
v=\limsup _{N \rightarrow s \infty}\left(\#\left\{k: n_{k}=N\right\}\right)^{\frac{1}{|N|}} .
$$

Then the following hold:

(L2) If $v>\varphi^{2}$, then all devious double covers $A\left(\tilde{S}_{h}, \tilde{\alpha}_{h}\right)$ have ergodic translation flow.

(L3) If $v<\varphi^{2}$, then all devious double covers $A\left(\tilde{S}_{h}, \tilde{\alpha}_{h}\right)$ have non-ergodic translation flow.

Our first order of business in proving this theorem is to characterize the devious double covers $A\left(\tilde{S}_{h}, \tilde{\alpha}_{h}\right)$ of $A\left(S_{L}, \alpha_{L}\right)$. We will prove the following:

Lemma 43 (Criterion for deviousness). In the context of the theorem above, suppose that $\left\langle n_{k}\right\rangle$ limits to $s \infty$ with $s \in\{ \pm 1\}$. Let $A \in S L_{ \pm}(2, \mathbb{R})$ and $h \in \operatorname{Hom}\left(\Gamma, \mathbb{Z}_{2}\right)$ be chosen so that $h \neq \mathbf{0}$, where $\mathbf{0} \in \operatorname{Hom}\left(\Gamma, \mathbb{Z}_{2}\right)$ denotes the trivial homomorphism. Then, the cover $A\left(\tilde{S}_{h}, \tilde{\alpha}_{h}\right)$ of $A\left(S_{L}, \alpha_{L}\right)$ is devious if and only if

$$
\lim _{m \rightarrow+\infty} \tau_{*}^{-s m}(h)=\mathbf{0} .
$$

Remark 44 (Reduction to case $s=-1$ ). It suffices to prove Lemma 43 in the case of $s=-1$. To see this fix $A$ and suppose $\lim n_{k}=+\infty$. Observe that $A\left(\tilde{S}_{h}, \tilde{\alpha}_{h}\right)$ is a devious double cover of $A\left(S_{L}, \alpha_{L}\right)$ if and only if $A^{\prime}\left(\tilde{S}_{\psi_{*}(h)}, \tilde{\alpha}_{\psi_{*}(h)}\right)$ is a devious double cover of $A^{\prime}\left(S_{L}, \alpha_{L}\right)$, since the surfaces are translation equivalent. Let $A^{\prime}=D(\psi) A$ and $h^{\prime}=\psi_{*}(h)$. Use $A^{\prime}$ to define the coding walk $\left\langle n_{k}^{\prime}\right\rangle$. Because of the action of $D(\psi)$ on regions (see (31) and (33)), we have $n_{k}^{\prime}=-n_{k}$ so that $\lim n_{k}=-\infty$. Finally observe that $\lim _{m \rightarrow \infty} \tau_{*}^{-m}(h)=\mathbf{0}$ if and only if $\lim _{n \rightarrow \infty} \tau_{*}^{-n}\left(h^{\prime}\right)=\mathbf{0}$ since

$$
\tau_{*}^{-n}\left(h^{\prime}\right)=\tau^{-n} \circ \psi_{*}(h)=\psi_{*} \circ \tau_{*}^{n}(h) \text { for all } n
$$

as the action factors through the Isom $(\mathbb{Z})$; see $(32)$. Similar observations demonstrate that we can assume $s=-1$ in the proof of Theorem 42 as well.

One direction of Lemma 43 is fairly easy: 
Proof of the "only if" part of Lemma 43. From the remark above, we can assume $s=-1$. Observe that a cover $\left(\tilde{S}_{h^{\prime}}, \tilde{\alpha}_{h^{\prime}}\right)$ is connected if and only if $h^{\prime} \neq \mathbf{0}$. So, assume $\lim n_{k}=-\infty$ and there is an $h^{\prime} \neq \mathbf{0}$ which is an accumulation point of $\tau_{*}^{m}(h)$ as $m \rightarrow+\infty$. Let $m(j)$ be an increasing sequence of integers so that $\tau_{*}^{m(j)}(h) \rightarrow h^{\prime}$. We will show that $g^{t} A\left(\tilde{S}_{h}, \tilde{\alpha}_{h}\right)$ has a connected accumulation point in $\tilde{\mathcal{O}}_{\mathbb{Z}_{2}}\left(S_{L}, \alpha_{L}\right)$.

Since the walk $\left\langle n_{k}\right\rangle$ tends to $-\infty$, for sufficiently large integers $j$, there is a $k(j)$ so that $n_{k(j)}=-m(j)$. From Lemma 40 , there is a sequence of times $t^{j}$ taken from the intervals associated to $n_{k(j)}$, a compact subset $K \subset S L_{ \pm}(2, \mathbb{R})$, and $M_{j} \in K$ so that

$$
g^{t^{j}} A\left(\tilde{S}_{h}, \tilde{\alpha}_{h}\right)=M_{j}\left(\tilde{S}_{\tau_{*}^{-n} k(j)},\left(\tilde{\alpha}_{\tau_{*}-n_{k(j)}(h)}\right) .\right.
$$

Since $n_{k(j)}=-m(j)$ we know $\lim _{k \rightarrow \infty} \tau_{*}^{-n_{k(j)}}(h)=h^{\prime}$. Since $K$ is compact, we find a subsequence $M_{j(i)}$ converging to some $M \in K$. Then

$$
\lim _{i \rightarrow \infty} M_{j(i)}\left(\tilde{S}_{\tau_{*}^{-n}{ }_{k o j(i)}(h)}, \tilde{\alpha}_{\tau_{*}^{-n} k_{0 j(i)}(h)}\right)=M\left(\tilde{S}_{h^{\prime}}, \tilde{\alpha}_{h^{\prime}}\right) \quad \text { in } \tilde{\mathcal{O}}\left(S_{L}, \alpha_{L}\right) .
$$

This is our desired connected accumulation point.

The other direction is more difficult in part because we do not know if $V^{\prime}=V\left(S_{L}, \alpha_{L}\right)$. We need to show that if $\lim _{m \rightarrow+\infty} \tau_{*}^{-s m}(h)=\mathbf{0}$ then every accumulation point of $g^{t} A\left(\tilde{S}_{h}, \tilde{\alpha}_{h}\right)$ in $\tilde{\mathcal{O}}_{\mathbb{Z}_{2}}\left(S_{L}, \alpha_{L}\right)$ is disconnected. But $\tilde{\mathcal{O}}_{\mathbb{Z}_{2}}\left(S_{L}, \alpha_{L}\right)$ is a quotient of $S L(2, \mathbb{R}) \times \operatorname{Cov}_{\mathbb{Z}_{2}}\left(S_{L}, \alpha_{L}\right)$ by $V\left(S_{L}, \alpha\right)$ which we do not know.

Fortunately, we are restricting attention to $A \in S L(2, \mathbb{R})$ so that $g^{t} A V^{\prime}$ is non-divergent in $S L_{ \pm}(2, \mathbb{R}) / V^{\prime}$. Then the geodesic $g^{t} A V^{\prime}$ is asymptotic to the convex core. We will use this and a compactness argument to argue that if $g^{t_{i}} A\left(\tilde{S}_{h}, \tilde{\alpha}_{h}\right)$ converges then there is a subsequence converging to a disconnected cover. Assuming that the space $\tilde{\mathcal{O}}_{\mathbb{Z}_{2}}\left(S_{L}, \alpha_{L}\right)$ is Hausdorff, the two limit points must be the same showing that every accumulation point is disconnected. So we need:

Proposition 45. The space $\tilde{\mathcal{O}}_{\mathbb{Z}_{2}}\left(S_{L}, \alpha_{L}\right)$ is Hausdorff.

Proof. Let $A\left(\tilde{S}_{h}, \tilde{\alpha}_{h}\right)$ and $A^{\prime}\left(\tilde{S}_{h^{\prime}}, \tilde{\alpha}_{h^{\prime}}\right)$ be distinct points in $\tilde{\mathcal{O}}_{\mathbb{Z}_{2}}\left(S_{L}, \alpha_{L}\right)$. We will find an open sets that isolate them from each other.

Observe that there is a natural continuous map $\tilde{\mathcal{O}}_{\mathbb{Z}_{2}}\left(S_{L}, \alpha_{L}\right) \rightarrow \mathcal{O}\left(S_{L}, \alpha_{L}\right)$ which sends $A\left(\tilde{S}_{h}, \tilde{\alpha}_{h}\right)$ to the quotient of $A\left(\tilde{S}_{h}, \tilde{\alpha}_{h}\right)$ by its translation automorphisms. Since all double covers are regular and $\left(S_{L}, \alpha_{L}\right)$ has no translation automorphisms, there is always a $\mathbb{Z}_{2}$ action on $A\left(\tilde{S}_{h}, \tilde{\alpha}_{h}\right)$ by its translation automorphisms. If under this map $A\left(\tilde{S}_{h}, \tilde{\alpha}_{h}\right)$ and $A^{\prime}\left(\tilde{S}_{h^{\prime}}, \tilde{\alpha}_{h^{\prime}}\right)$ project to different points, then we can build disjoint open sets by lifting disjoint open sets from $\mathcal{O}\left(S_{L}, \alpha_{L}\right)$. Recall that $\mathcal{O}\left(S_{L}, \alpha_{L}\right)$ is naturally identified with $S L_{ \pm}(2, \mathbb{R}) / V\left(S_{L}, \alpha_{L}\right)$ which is Hausdorff because $V\left(S_{L}, \alpha_{L}\right)$ is discrete by Proposition 32 .

Now suppose that $A\left(\tilde{S}_{h}, \tilde{\alpha}_{h}\right)$ and $A^{\prime}\left(\tilde{S}_{h^{\prime}}, \tilde{\alpha}_{h^{\prime}}\right)$ both cover $A\left(S_{L}, \alpha_{L}\right)$. By left multiplying both surfaces by $A^{-1}$ we can assume without loss of generality that both surfaces cover $\left(S_{L}, \alpha_{L}\right)$. Then they both lie in the $\operatorname{Cov}_{\mathbb{Z}_{2}}\left(S_{L}, \alpha_{L}\right)$. Recall $\operatorname{Cov}_{\mathbb{Z}_{2}}\left(S_{L}, \alpha_{L}\right)=\operatorname{Hom}\left(\Gamma, \mathbb{Z}_{2}\right)$; see (25). This space is a Cantor set and so is Hausdorff. So, we can find open sets $U_{1}$ and $U_{2}$ that isolate the two surfaces within $\operatorname{Cov}_{\mathbb{Z}_{2}}\left(S_{L}, \alpha_{L}\right)$. Since the Veech groups are discrete, we can find a neighborhood $N \subset S L_{ \pm}(2, \mathbb{R})$ so that no non-trivial element of $V\left(S_{L}, \alpha_{L}\right)$ has the form $B_{1}^{-1} B_{2}$ where $B_{1}, B_{2} \in N$. We claim that the images of $N \times U_{1}$ and $N \times U_{2}$ are disjoint in $\tilde{\mathcal{O}}_{\mathbb{Z}_{2}}\left(S_{L}, \alpha_{L}\right)$. Let $B_{1}\left(S_{h_{1}}, \alpha_{h_{1}}\right)$ and $B_{2}\left(S_{h_{2}}, \alpha_{h_{2}}\right)$ be two surfaces taken from these 
neighborhoods. Then they are translation equivalent if and only if $\left(S_{h_{1}}, \alpha_{h_{1}}\right)$ is equivalent to $B_{1}^{-1} B_{2}\left(S_{h_{2}}, \alpha_{h_{2}}\right)$. If they are translation equivalent, we must have $B_{1}^{-1} B_{2}=I$ from arguments in the previous paragraph together with the definition of $N$. But $\left(S_{h_{1}}, \alpha_{h_{1}}\right) \in U_{1}$ and $\left(S_{h_{2}}, \alpha_{h_{2}}\right) \in U_{2}$, so they can not be translation equivalent.

The following will finish our proof of Lemma 43 .

Proof of the "if" part of Lemma [43. Suppose that $\lim n_{k}=-\infty$ and $\lim _{m \rightarrow+\infty} \tau_{*}^{m}(h)=\mathbf{0}$. We must show that the cover $A\left(\widetilde{S}_{h}, \tilde{\alpha}_{h}\right)$ of $A\left(S_{L}, \alpha_{L}\right)$ is devious.

We will show every $\omega$-limit point of $g^{t} A\left(\tilde{S}_{h}, \tilde{\alpha}_{h}\right)$ in $\tilde{\mathcal{O}}_{\mathbb{Z}_{2}}\left(S_{L}, \alpha_{L}\right)$ is disconnected. Suppose that $t_{i}$ is a sequence of times tending to $+\infty$ and

$$
\lim _{i \rightarrow \infty} g^{t_{i}} A\left(\tilde{S}_{h}, \tilde{\alpha}_{h}\right)=B\left(\tilde{S}_{h^{\prime}}, \tilde{\alpha}_{h^{\prime}}\right) \quad \text { in } \tilde{O}_{\mathbb{Z}_{2}}\left(S_{L}, \alpha_{L}\right)
$$

Consider the sequence of points $p_{i}=O(2) g^{t_{i}} A V^{\prime}$ in $O(2) \backslash S L_{ \pm}(2, \mathbb{R}) / V^{\prime}$. We claim that there is a cusp neighborhood $U$ of $O(2) \backslash S L_{ \pm}(2, \mathbb{R}) / V^{\prime}$ (given by $\operatorname{Im}(z)>c$ for some $c \geq 2$ in the fundamental domain of Figure 5) so that $p_{i} \notin U$ for all $i$. Otherwise there is a subsequence where $p_{i_{j}}$ exists the cusp. However, in this case the injectivity radius of the surface $g^{t_{i_{j}}} A\left(S_{L}, \alpha_{L}\right)$ would tend to zero, because $g^{t_{i_{j}}} A$ would contract the circumferences of some cylinder decomposition (which up the action of $V^{\prime}$ is vertical) more an more as we move up the cusp, and thus $g^{t_{i}} A\left(S_{L}, \alpha_{L}\right)$ would have injectivity radius tending to zero as $j \rightarrow \infty$. For the same reason, the injectivity radius of the surfaces $g^{t_{i j}} A\left(\tilde{S}_{h} \tilde{\alpha}_{h}\right)$ would have to tend to zero. But this contradicts that this sequence limits to $B\left(\tilde{S}_{h^{\prime}}, \tilde{\alpha}_{h^{\prime}}\right)$.

Let $C_{d}$ be a $d$-neighborhood of the compact core of $O(2) \backslash S L_{ \pm}(2, \mathbb{R}) / V^{\prime}$. Since by hypothesis $g^{t} A V^{\prime}$ is non-divergent in $S L_{ \pm}(2, \mathbb{R}) / V^{\prime}$, we know that this trajectory is asymptotic to the convex core. Combining this with this the previous paragraph, we see that

$$
O(2) g^{t_{i}} A V^{\prime} \in C_{d} \backslash U \text { for } i \text { sufficiently large. }
$$

By compactness of the fiber over $C_{d} \backslash U$, we can assume that after passing to a subsequence that $g^{t_{i}} A V^{\prime}$ converges in $S L_{ \pm}(2, \mathbb{R}) / V^{\prime}$.

Now consider the sequence $g^{t_{i}} A \tilde{V}^{\prime}$ in $S L_{ \pm}(2, \mathbb{R}) / \tilde{V}^{\prime}$. Let $k(i)$ be such that $t_{i} \in\left[t_{k(i)}, t_{k(i)+1}\right]$. Defining

$$
\tilde{p}_{i}=O(2) g^{t_{i}} A \tilde{V}^{\prime} \quad \text { we see } \quad \tilde{p}_{i} \in F_{n_{k(i)}} \text { for each } i,
$$

and so $\tau^{-n_{k(i)}}\left(\tilde{p}_{i}\right) \in F_{0}$ for each $i$ where $\tau^{-n_{k(i)}}$ is acting as an element of the deck group of the covering (30) as in (34). Since each point of $O(2) \backslash S L_{ \pm}(2, \mathbb{R}) / V^{\prime}$ has only two lifts to $F_{0}$, we see that by passing to a subsequence we can assume $\tau^{-n_{k(i)}}\left(g^{t_{i}} A \tilde{V}^{\prime}\right)$ converges in $S L_{ \pm}(2, \mathbb{R}) / \tilde{V}^{\prime}$ above $F_{0}$. Recalling how $\tau$ acts (see $(32)$ and $(34)$ ), this means there is a sequence $R_{i} \in \tilde{V}^{\prime}$ so that

$$
g^{t_{i}} A D(\rho \circ \psi)^{n_{k(i)}} R_{i} \quad \text { converges to some limit } L \in S L_{ \pm}(2, \mathbb{R}) \text {. }
$$

Observe that in $\tilde{\mathcal{O}}_{\mathbb{Z}_{2}}\left(S_{L}, \alpha_{L}\right)$ we have

$$
\begin{aligned}
g^{t_{i}} A\left(\tilde{S}_{h}, \tilde{\alpha}_{h}\right) & =g^{t_{i}} A D(\rho \circ \psi)^{n_{k(i)}} R_{i} \cdot\left(R_{i}^{-1} D(\rho \circ \psi)^{-n_{k(i)}}\left(\tilde{S}_{h}, \tilde{\alpha}_{h}\right)\right) \\
& =g^{t_{i}} A D(\rho \circ \psi)^{n_{k(i)}} R_{i}\left(\tilde{S}_{\tau_{*}^{-n} k_{k(i)}(h)}, \tilde{\alpha}_{\tau_{*}^{-n_{k(i)}(h)}}\right) .
\end{aligned}
$$


The $S L_{ \pm}(2, \mathbb{R})$ part of this last expression converges to $L$. The surface part converges to $\left(\tilde{S}_{\mathbf{0}}, \tilde{\alpha}_{\mathbf{0}}\right)$ because $\lim n_{k}=-\infty$ and $\lim _{m \rightarrow+\infty} \tau_{*}^{m}(h)=\mathbf{0}$. So, because $\tilde{O}_{\mathbb{Z}_{2}}\left(S_{L}, \alpha_{L}\right)$ is Hausdorff by Proposition 45, we must have

$$
B\left(\tilde{S}_{h^{\prime}}, \tilde{\alpha}_{h^{\prime}}\right)=\lim _{i \rightarrow \infty} g^{t_{i}} A\left(\tilde{S}_{h}, \tilde{\alpha}_{h}\right)=L\left(\tilde{S}_{\mathbf{0}}, \tilde{\alpha}_{\mathbf{0}}\right)
$$

In particular, $B\left(\tilde{S}_{h^{\prime}}, \tilde{\alpha}_{h^{\prime}}\right)$ is disconnected.

Now we will study devious covers by studying those $h$ so that $\tau_{*}^{m}(h)$ decays to $\mathbf{0}$. A formula for $\tau_{*}$ is given below.

Proposition 46. The action of translation by one on $\operatorname{Hom}\left(\Gamma, \mathbb{Z}_{2}\right)$ is given by

$$
\left(\tau_{*}(h)\right)(i)= \begin{cases}h(-i)+h(i-1)+h(i)+h(i+1) & \text { if } i<-2, \\ h(-i)+h(-2)+h(-3) & \text { if } i=-2, \\ h(-i)+h(-2) & \text { if } i=-1, \\ h(-i) & \text { if } i>0 .\end{cases}
$$

The action of translation by negative one is given by:

$$
\left(\tau_{*}^{-1}(h)\right)(i)= \begin{cases}h(-i) & \text { if } i<0, \\ h(-i)+h(2) & \text { if } i=1, \\ h(-i)+h(2)+h(3) & \text { if } i=2, \\ h(-i)+h(i-1)+h(i)+h(i+1) & \text { if } i>2 .\end{cases}
$$

Proof. This follows from the fact that $\tau=\delta \circ D(\rho \circ \psi)$ and $\tau^{-1}=\delta \circ D(\psi \circ \rho)$; see equation 31. The actions of $\psi$ and $\rho$ on $\operatorname{Hom}\left(\Gamma, \mathbb{Z}_{2}\right)$ are given in equations 26 and 27 , respectively.

To analyze the case when $\lim _{m \rightarrow+\infty} \tau_{*}^{m}(h)=\mathbf{0}$, we introduce the proximity function which measures how close a homomorphism is to the trivial homomorphism $\mathbf{0}$ :

$$
P: \operatorname{Hom}\left(\Gamma, \mathbb{Z}_{2}\right) \rightarrow\{1,2,3, \ldots, \infty\} ; \quad P(h)= \begin{cases}\infty & \text { if } h=\mathbf{0}, \\ \min \{|i|: h(i) \neq 0\} & \text { otherwise }\end{cases}
$$

Observe a sequence $h_{n}$ tends to 0 if and only if $P\left(h_{n}\right) \rightarrow \infty$.

For each integer $k \geq 0$, we define a cylinder set in $\operatorname{Hom}\left(\Gamma, \mathbb{Z}_{2}\right)$ by

$$
C_{k}=\{h: h(k)=h(-k-1)=1 \text { and } h(i)=0 \text { when }-k-1<i<k\} .
$$

Note that $h \in C_{k}$ implies $P(h)=k$. These cylinder sets turn out to be very important, for understanding which elements of $\operatorname{Hom}\left(\Gamma, \mathbb{Z}_{2}\right)$ are limit to the trivial homomorphism $\mathbf{0}$ under translation.

Lemma 47. The collection of $h \in \operatorname{Hom}\left(\Gamma, \mathbb{Z}_{2}\right)$ so that $\lim _{n \rightarrow+\infty} \tau_{*}^{n}(h)=\mathbf{0}$ is given by

$$
\{\mathbf{0}\} \cup \bigcup_{j \geq 0} \tau_{*}^{-j}(Z) \text { where } Z=\bigcup_{k \geq 2} \bigcap_{m \geq 0} \tau_{*}^{-m}\left(C_{k+m}\right) .
$$

Furthermore, for any function $f:\{i: i \geq 2\} \rightarrow \mathbb{Z}_{2}$ which is not identically zero, there is an $h \in Z$ so that $h(i)=f(i)$ for all $i \geq 2$. 
The second statement says that there are a number of elements of $\operatorname{Hom}\left(\Gamma, \mathbb{Z}_{2}\right)$ which limit on 0. (Informally, $Z$ has half the information entropy of $\operatorname{Hom}\left(\Gamma, \mathbb{Z}_{2}\right)$, or with an appropriate natural metric, $Z$ has half the Hausdorff dimension of $\operatorname{Hom}\left(\Gamma, \mathbb{Z}_{2}\right)$.) This together with Lemma 43 prove the existence of devious covers as stated in Theorem 42 , b

The following proposition is the main ingredient in the proof of the lemma above.

Proposition 48. (1) If $k \geq 2$ and $h \in C_{k}$, then $P\left(\tau_{*}(h)\right)=k+1$.

(2) If $k \geq 2$ is an integer, then $\tau_{*}^{-1}\left(C_{k+1}\right) \subset C_{k}$.

(3) If $k \geq 2, P(h)=k$ and $h \notin C_{k}$, then either $P\left(\tau_{*}(h)\right)=k-1$ and $\tau_{*}(h) \notin C_{k-1}$ or $P\left(\tau_{*}^{2}(h)\right)=k-1$ and $\tau_{*}^{2}(h) \notin C_{k-1}$.

Proof. We prove each of the statements below.

(1) Suppose that $h \in C_{k}$ and $k \geq 2$. Then $h(k)=h(-k-1)=1$ while $h(i)=0$ for $-k \leq i \leq k-1$. Then $P\left(\tau_{*}(h)\right) \leq k+1$, because $\tau_{*}(h)(k+1)=h(-k-1)=1$ by Proposition 46. For $0<i \leq k$, we have $\tau_{*}(h)(i)=h(-i)=0$. Also observe that

$$
\tau_{*}(h)(-k)=h(k)+h(-k-1)=1+1=0,
$$

while the other terms from Proposition 46 vanish. Finally, all terms in the expression for $\tau_{*}(h)(i)$ vanish when $-k<i<0$. Therefore, $P\left(\tau_{*}(h)\right)=k+1$ as claimed.

(2) Suppose $h \in C_{k+1}$ with $k \geq 2$. Then $h(k+1)=h(-k-2)=1$ while $h(i)=0$ for $-k-1 \leq i \leq k$. By Proposition 46, we see that $\tau_{*}^{-1}(h)(-k-1)=h(k+1)=1$. Also,

$$
\tau_{*}^{-1}(h)(k)= \begin{cases}h(-k)+h(2)+h(3)=0+0+1=1 & \text { if } k=2, \\ h(-k)+h(k-1)+h(k)+h(k+1)=0+0+0+1=1 & \text { if } k>2 .\end{cases}
$$

Now suppose $-k \leq i<0$. Here we have $\tau_{*}^{-1}(h)(i)=h(-i)=0$. Finally consider the case when $0<i<k$. All terms vanish from the expression for the expression $\tau_{*}^{-1}(h)(i)$ given in Proposition 46, so $\tau_{*}^{-1}(h)(i)=0$. Taken together, we see $\tau_{*}^{-1}(h) \in C_{k}$.

(3) Suppose $k \geq 2, P(h)=k$ and $h \notin C_{k}$. Since $P(h)=k$, we know that $h(i)=0$ when $|i|<k$. Observe that the set of $h$ with $P(h)=k$ and $h \notin C_{k}$ is the union of the two pieces:

$$
\begin{gathered}
A=\{h: P(h)=k \text { and } h(-k)=1\}, \\
B=\{h: P(h)=k, h(-k)=0, h(k)=1, \text { and } h(-k-1)=0\} .
\end{gathered}
$$

First assume that $h \in A$ so that $h(-k)=1$ but $h(i)=0$ when $|i|<k$. Then, $\tau_{*}^{1}(h)(-k+1)$ is given by one of the expressions

$$
\begin{cases}h(k-1)+h(-k)+h(-k+1)+h(-k+2)=0+1+0+0=1 & \text { if } k>3, \\ h(k-1)+h(-2)+h(-3)=0+0+1=1 & \text { if } k=3, \\ h(k-1)+h(-2)=0+1=1 & \text { if } k=2 .\end{cases}
$$

Since proximity can decrease by at most one when applying $\tau_{*}^{1}$, we see $P\left(\tau_{*}^{1}(h)\right)=k-1$. Also, we have by definition of $C_{k-1}$ that $\tau_{*}^{1}(h) \notin C_{k-1}$. Now consider the case when $h \in B$. Then $h(i)=0$ when $-k-1 \leq i<k$ and $h(k)=1$. In this case $\tau_{*}^{1}(h)(i)=0$ when $|i|<k-1$ since proximity can decrease by at most one. We observe $\tau_{*}^{1}(h)(k-1)=h(-k+1)=0$. All terms vanish in the expression for $\tau_{*}^{1}(h)(-k+1)$ given by Proposition 46 , so $\tau_{*}^{1}(h)(-k+1)=0$. On the other hand,

$$
\tau_{*}^{1}(h)(-k)= \begin{cases}h(k)+h(-k-1)+h(-k)+h(-k+1)=1+0+0+0=1 & \text { if } k>2, \\ h(k)+h(-2)+h(-3)=1+0+0=1 & \text { if } k=2 .\end{cases}
$$


Since $\tau_{*}^{1}(h)(-k)=1$, we see that $\tau_{*}^{1}(h) \in A$. Because of our discussion of what happens for elements of $A$, we see that $P\left(\tau_{*}^{2}(h)\right)=k-1$ and $\tau_{*}^{2}(h) \notin C_{k-1}$.

In the proof of Lemma 47, it is useful to note the following Corollary to the Proposition above.

Corollary 49. Let $k \geq 1$ be an integer. If $P(h)=k$ but $h \notin C_{k}$, then there is an $n \geq 0$ so that $P\left(\tau_{*}^{n}(h)\right)=1$.

Proof. We prove this by induction in $k$. This holds with $n=0$ when $k=1$. When $k=2$, this follows from statement (3) of Proposition 48. Now let $k \geq 3$ and suppose the conclusion holds when $P(h)=k-1$ and $h \notin C_{k-1}$. Suppose $P(h)=k$ and $h \notin C_{k}$. Again using statement (3) of Proposition 48, we see that either $P\left(\tau_{*}(h)\right)=k-1$ and $\tau_{*}(h) \notin C_{k-1}$ or $P\left(\tau_{*}^{2}(h)\right)=k-1$ and $\tau_{*}^{2}(h) \notin C_{k-1}$. Using our induction hypothesis applied to these cases, we see the conclusion holds, and the whole statement holds by induction.

Proof of Lemma 47. Let $Z=\bigcup_{k \geq 2} \bigcap_{m \geq 0} \tau_{*}^{-m}\left(C_{k+m}\right)$ as in the theorem. Let $h \in Z$. Then there is a $k \geq 2$ so that $\tau_{*}^{m}(h) \in C_{k+m}$ for all $m \geq 0$. Then $P\left(\tau_{*}^{m}(h)\right)=k+m$ tends to $\infty$ as $m \rightarrow \infty$. Thus, $\lim _{m \rightarrow \infty} \tau_{*}^{m}(h)=\mathbf{0}$. From this it follows that everything in the set listed in the theorem, $\{\mathbf{0}\} \cup \bigcup_{j \geq 0} \tau_{*}^{-j}(Z)$, limits to $\mathbf{0}$.

Conversely, suppose $h \in \operatorname{Hom}\left(\Gamma, \mathbb{Z}_{2}\right)$ satisfies $\lim _{n \rightarrow \infty} \tau_{*}^{n}(h)=\mathbf{0}$ and $h \neq \mathbf{0}$. We know that $P\left(\tau_{*}^{n}(h)\right) \rightarrow \infty$ as $n \rightarrow \infty$. In particular, we see that for any $k \geq 1$, there are only finitely many $n$ so that

$$
P\left(\tau_{*}^{n}(h)\right)=k .
$$

Let $K \geq 2$ be the smallest value of $k$ larger than one so that the above equation has a solution for $n$, and let $N$ be the maximal $n$ satisfying the equation when $k=K$. We claim that

$$
\tau_{*}^{N}(h) \in \bigcap_{n \geq 0} \tau_{*}^{-n}\left(C_{K+n}\right) .
$$

Otherwise, there is a smallest $n \geq 0$ so that $\tau_{*}^{N+n}(h) \notin C_{K+n}$. Then by the corollary above, we see that there is an $m \geq N+n$ so that $P\left(\tau_{*}^{m}(h)\right)=1$. But since $P\left(\tau_{*}^{n}(h)\right)$ tends to $\infty$ and as $n$ increases the proximity can increase by at most one, we see that there is an $m^{\prime}>m$ so that $(38)$ is satisfied for $n=m^{\prime}$. But this contradicts the definition of $N$. Therefore, (39) is true after all. Observe that the equation implies that $\tau_{*}^{N}(h) \in Z$, and thus $h \in \bigcup_{j \geq 0} \tau_{*}^{-j}(Z)$ as desired.

It remains to prove the last sentence of the theorem which guarantees there are a lot of $h$ so that $\lim _{n \rightarrow \infty} \tau_{*}^{n}(h)=\mathbf{0}$. A main point here is that for each $k \geq 2$, the set $A_{k}=\bigcap_{m>0} \tau_{*}^{-m}\left(C_{k+m}\right)$ is non-empty. Indeed, as continuous images of cylinder sets, each $\tau_{*}^{-m}\left(C_{k+m}\right)$ is compact. The sets being intersected are nested in the sense that

$$
\tau_{*}^{-m-1}\left(C_{k+m+1}\right) \subset \tau_{*}^{-m}\left(C_{k+m}\right)
$$

by statement (2) of the lemma. Therefore, we can make a choice of an $\tilde{h}_{k} \in A_{k}$ for every $k \geq 2$.

Now suppose that $f:\{i \in \mathbb{Z}: i \geq 2\} \rightarrow \mathbb{Z}_{2}$ is defined and not identically zero. Let $k=\min \{i: f(i) \neq 0\}$. We will inductively define a sequence of functions $h_{j} \in \operatorname{Hom}\left(\Gamma, \mathbb{Z}_{2}\right)$ for $j \geq k$ so that the sequence converges to an extension of $f$. Our functions will all lie in 
$A_{k}$. Since $A_{k}$ is closed, this will suffice to prove that the limiting function lies in $A_{k}$. We will also ensure that

$$
h_{j}(i)=f(i) \quad \text { when } 2 \leq i \leq j .
$$

To ensure convergence of the sequence $h_{j}$, we will also have that

$$
h_{j}(i)=h_{j-1}(i) \quad \text { when }|i|<j .
$$

Observe that $f(k)=1$, thus we can define $h_{k}=\tilde{h}_{k} \in A_{k}$. This serves as our base case. Now assume that $j>k$ and $h_{j-1}$ is defined and satisfies the hypotheses above. We will define $h_{j}$. If $h_{j-1}(j)=f(j)$, then we can take $h_{j}=h_{j-1}$. Otherwise, we define $h_{j}=h_{j-1}+\tilde{h}_{j}$. Since $\tilde{h}_{j} \in C_{j}$, we know that $\tilde{h}_{j}(j)=1$. Therefore, we must have $h_{j}(j)=f(j)$. When $|i|<j$, we have $\tilde{h}_{j}(i)=0$ because $h_{j} \in C_{j}$, therefore

$$
h_{j}(i)=h_{j-1}(i) \quad \text { when }|i|<j
$$

by inductive hypothesis. This simultaneously ensures both equation (41) holds and verifies equation (40) for $i<j$ since by hypothesis $h_{j-1}(i)=f(i)$ when $2 \leq i \leq j-1$. From the inductive hypothesis and definition of $\tilde{h}_{j}$, we have

$$
\tau_{*}^{m}\left(h_{j-1}\right) \in C_{k+m} \quad \text { and } \quad \tau_{*}^{m}\left(\tilde{h}_{j}\right) \in C_{j+m}
$$

for each integer $m \geq 0$. Observe that when $j>k$, the sum of an element in $C_{k+m}$ and $C_{j+m}$ lies in $C_{k+m}$. Therefore, we see by linearity of $\tau_{*}^{m}$ that

$$
\tau_{*}^{m}\left(h_{j}\right)=\tau_{*}^{m}\left(h_{j-1}\right)+\tau_{*}^{m}\left(\tilde{h}_{j}\right) \in C_{k+m}
$$

for every integer $m \geq 0$. Thus, $h_{j} \in A_{k}$ as desired. This completes the inductive step.

Proof of statement (L2) of Theorem 42. We will consider the translation flow on the devious double cover $A\left(\tilde{S}_{h}, \tilde{\alpha}_{h}\right)$ of $A\left(S_{L}, \alpha_{L}\right)$ under several assumptions. We assume the trajectory $g^{t} A V^{\prime}$ is non-divergent in $S L_{ \pm}(2, \mathbb{R}) / V^{\prime}$. From the geodesic $g^{t} A \tilde{V}^{\prime}$, we define the coding walk $\left\{n_{k}\right\}$. We assume that the number of visits to any integer is finite, but that the growth exponent of the visit count satisfies $v>\varphi^{2}$. As allowed by Remark 44, we assume that $\lim _{k \rightarrow \infty} n_{k}=-\infty$. By Lemma 43, we know that $\lim _{m \rightarrow+\infty} \tau_{*}^{m}(h)=\mathbf{0}$. In particular, $\lim _{k \rightarrow \infty} \tau_{*}^{-n_{k}}(h)=\mathbf{0}$.

We will prove ergodicity holds for the translation flow on $A\left(\tilde{S}_{h}, \tilde{\alpha}_{h}\right)$ by appealing to Theorem 19. (We upgrade to unique ergodicity at the end of the proof.) Let $\eta>0$. We will find subsurfaces of $X_{t}=g^{t} A\left(\tilde{S}_{h}, \tilde{\alpha}_{h}\right)$ and related geometric quantities so that the integral (14) is infinite.

Now recall the definitions of the coding walk; see Proposition 39. There is a sequence of times $t_{k}$ so that

$$
g^{t} A \tilde{V}^{\prime} \in F_{n_{k}} \quad \text { when } t_{k}<t<t_{k+1} .
$$

Let $U$ and $C_{d}$ be subsets of $O(2) \backslash S L_{ \pm}(2, \mathbb{R}) / V^{\prime}$ as in Lemma 40 . Let

$$
J_{k}=\left\{t \in\left(t_{k}, t_{k+1}\right): O(2) g^{t} A V^{\prime} \in C_{d} \backslash U\right\} .
$$

Since the geodesic $O(2) g^{t} A V^{\prime}$ is non-divergent it is asymptotic to the convex core and so there is a $\mathrm{j}>0$ so that the constant

$$
\mathrm{j} \geq \operatorname{length}\left(J_{k}\right) \quad \text { for } k \text { sufficiently large. }
$$




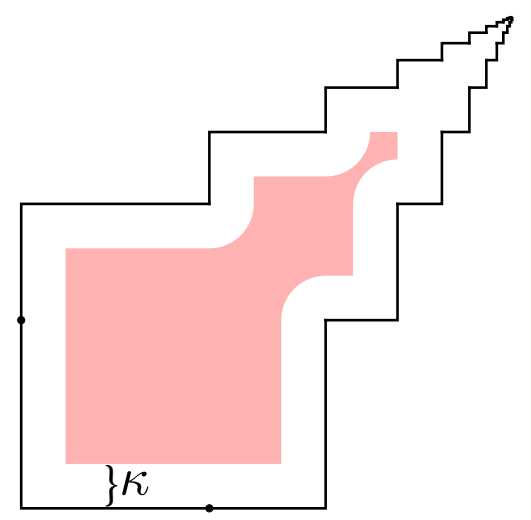

Figure 7. An example $U(\kappa)$ in the disk making up $\left(S_{L}, \alpha_{L}\right)$.

(In fact, length $\left(J_{k}\right)$ has a uniform lower bound once the geodesic is within distance $d$ from the convex core.) By Lemma 40 , there is a compact set $K \subset S L_{ \pm}(2, \mathbb{R})$ so that for any $t \in J_{k}$ there is a matrix $M_{t} \in K$ so that

$$
X_{t}=M_{t}\left(\tilde{S}_{\tau_{*}^{-n}(h)}, \tilde{\alpha}_{\tau_{*}^{-n_{k}}(h)}\right) \quad \text { as elements of } \tilde{\mathcal{O}}_{\mathbb{Z}_{2}}\left(S_{L}, \alpha_{L}\right) .
$$

We will now explain how to find subsurfaces. The surfaces will always be obtained by lifting two copies of a disk in $\left(S_{L}, \alpha_{L}\right)$; so we will always have $C_{t}=2$ in the language of the Theorem 19. We think of $\left(S_{L}, \alpha_{L}\right)$ as depicted by Figure 4 , the surface is a topological disk in the plane with edge identifications. For small $\kappa>0$, consider the subset $U_{0}(\kappa)$ of this disk consisting of points whose distance from the boundary of the disk is greater than $\kappa$. Then let $U(\kappa) \subset U_{0}(\kappa)$ be the subsurface of the largest area. Since these regions exhaust the disk as $\kappa \rightarrow 0$, we can choose a $\kappa$ so that $U=U(\kappa)$ contains more than a factor of $1-\eta$ of the disk's area. See Figure 7 for an example of $U(\kappa)$. We can think of $U$ as lying in the surface $\left(S_{L}, \alpha_{L}\right)$.

In order to define our surfaces $S_{t} \subset A\left(\tilde{S}_{h}, \tilde{\alpha}_{t}\right)$, observe that $A\left(\tilde{S}_{h}, \tilde{\alpha}_{t}\right)=g^{-t}\left(X_{t}\right)$ when $t \in \bigcup_{k} J_{k}$ and consider $X_{t}$ as in (43). (We will not bother to estimate the value being integrated when $t \notin \bigcup_{k} J_{k}$.) Let $U_{t}^{1}$ and $\tilde{U}_{t}^{2}$ be the two lifts of $U$ to $\left(\tilde{S}_{\tau_{*}^{-n} k_{(h)}}, \tilde{\alpha}_{\tau_{*}^{-n} k_{(h)}}\right)$. Then we define $S_{t}=g^{-t} M_{t}\left(\tilde{U}_{t}^{1} \cup \tilde{U}_{t}^{2}\right)$. Evaluating geometric quantities of $S_{t} \subset A\left(\tilde{S}_{h}, \tilde{\alpha}_{t}\right)$ with dist $_{t}$ is the same as evaluating the same quantities for the subsurface $M_{t}\left(\tilde{U}_{t}^{1} \cup \tilde{U}_{t}^{2}\right)$ of $X_{t}$. Observe that for $t \in \bigcup_{k} J_{k}$ :

- $C_{t}=2$.

- The distance from $M_{t}\left(\tilde{U}_{t}^{1} \cup \tilde{U}_{t}^{2}\right)$ to the singular set has a uniform lower bound, i.e., $\epsilon(t)$ can be taken to be a positive constant, since $\epsilon(t)$ is bounded by $\kappa$ divided by the operator norm of $M_{t}^{-1}$ which has a uniform upper bound since $M_{t} \in K$ and $K$ is compact.

- The diameters of the components have a uniform upper bound given by the diameter of $U$ times the maximal operator norm of $M \in K$. So $\mathcal{D}_{t}^{i}$ can be taken to be a constant independent of $t$ and $i$.

Finally, we need to consider the maximum over all curves joining our two subsurfaces of the

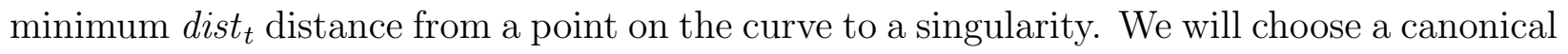
curve which depends mostly on the proximity of $\tau_{*}^{-n_{k}}(h)$ to zero, $P \circ \tau_{*}^{-n_{k}}(h)$, which was 


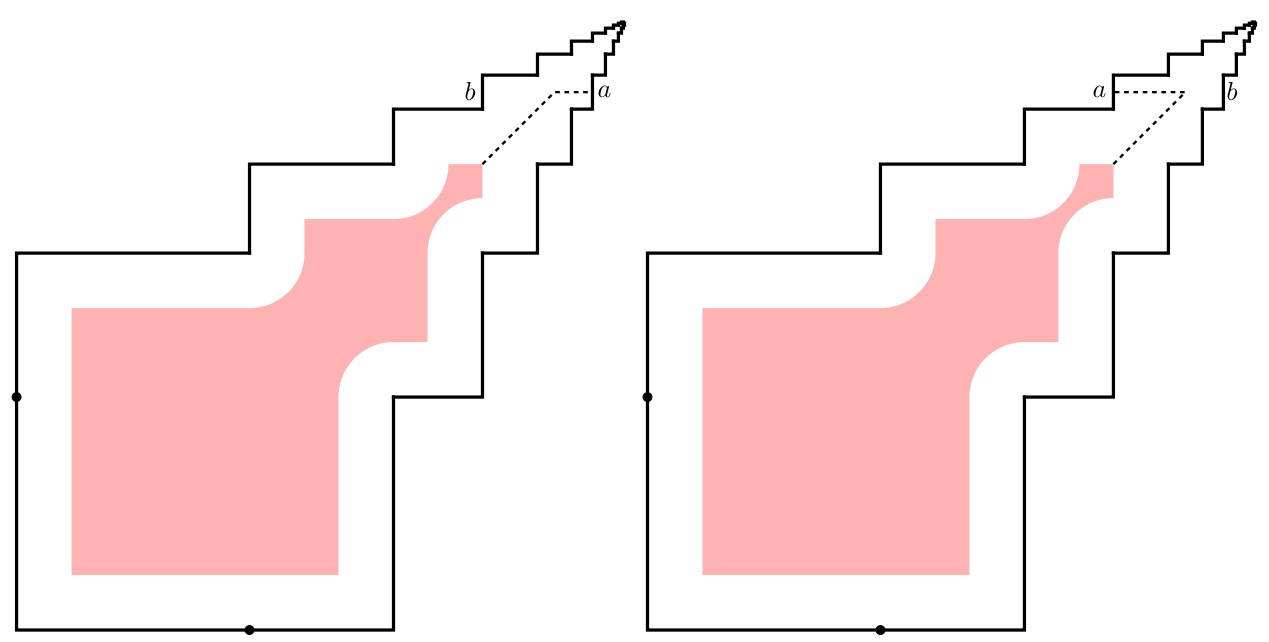

Figure 8. The path joining the two subsurfaces when $P\left(h_{*}\right)=5$ and $h_{*}(5)=$ 1. The letters $a$ and $b$ denote edges joining the two disks defining the cover in this case.

defined in equation (37). We will first define a curve joining $\tilde{U}_{t}^{1}$ and $\tilde{U}_{t}^{2}$ in $\left(\tilde{S}_{\tau_{*}^{-n_{k}(h)}}, \tilde{\alpha}_{\tau_{*}^{-n_{k}(h)}}\right)$, then we will push this curve under $M_{t}$ into $X_{t}$. (Again it suffices to measure things in $X_{t}$.)

Consider the cover $\left(\tilde{S}_{\tau_{*}^{-n_{k}(h)}}, \tilde{\alpha}_{\tau_{*}^{-n_{k}(h)}}\right)$ as a pair of infinite polygons with edges labeled as in Figure 4 and identified in some way. The edge labels with the smallest absolute values which are glued so as to join up the two polygons are of the form $p= \pm P \circ \tau_{*}^{-n_{k}}(h)$ in Figure 4. We choose a curve to leave the first subsurface and move upward along the slope 1 line of symmetry until it reaches the height of the edge $p$, then it changes trajectory by $\pm 45^{\circ}$ and travels through the midpoint of the edge connecting to the other disk. The curve continues until it hits the line of symmetry in the second disk, and it returns to the second subsurface along the line of symmetry of the disk. See Figure 8. It should be observed that there are positive constants $a$ and $b$ so that the distance from this curve to metric completion are of the form

$$
\min \left\{a, b \varphi^{-\left|P \circ \tau_{*}^{-n}(h)\right|}\right\} .
$$

(This is a consequence of the apparent self-similarity of the surface.) Then using the fact that $M_{t}$ is taken from a compact set, we see we can take

- $\delta_{t}=c \min \left\{a, b \varphi^{-\left|P \circ \tau_{*}^{-n}{ }_{k}(h)\right|}\right\}$ for some $c>0$.

We now have control of all the geometric quantities and can bound the integral. From the above definitions $d=\epsilon(t)^{-2} \sum_{i=1}^{2} \mathcal{D}_{t}^{i}$ is a positive constant. For $t \in J_{k}$, the quantity being integrated is of the form

$$
\left(d+\frac{1}{c \min \left\{a, b \varphi^{-\left|P \circ \tau_{*}^{-n_{k}}(h)\right|}\right\}}\right)^{-2} .
$$

Now we invoke the hypothesis that $\lim _{N \rightarrow \infty} \tau_{*}^{N}(h)=\mathbf{0}$. By Lemma 47 , we know that there is an $M>0$ and a $L \in \mathbb{Z}$ so that $\tau_{*}^{N}(h)$ lies in the cylinder set $C_{L+N}$ for all integers $N>M$. In particular, $P\left(\tau_{*}^{N}(h)\right)=L+N$. Then for sufficiently large values of $N$, say $N \geq N_{0}$, we can arrange that when $n_{k}=-N$,

$$
\min \left\{a, b \varphi^{-\left|P \circ \tau_{*}^{-n_{k}}(h)\right|}\right\}=b \varphi^{-N-L} .
$$


Let $V_{N}=\#\left\{k:-n_{k}=N\right\}$. Recall 42 , the length of each $J_{j}$ is bounded from below by $\mathrm{j}>0$. This allows us to write a lower bounds for the integral as

$$
\mathrm{J} \sum_{N=N_{0}}^{\infty} V_{N}\left(d+\frac{1}{b c} \varphi^{N+L}\right)^{-2} .
$$

An application of the root test tells us that this series diverges if

$$
v=\limsup _{N \rightarrow \infty} V_{N}>\varphi^{2} .
$$

This was precisely our hypothesis, and Theorem 19 gives us ergodicity.

We will now show that ergodicity implies unique ergodicity in statement (L2). By Corollary 3, we just need to know that the translation flow on $A\left(S_{L}, \alpha_{L}\right)$ is uniquely ergodic. By Theorem 34, we have unique ergodicity unless $O(2) g^{t} A V^{\prime}$ is asymptotic to the convex core boundary. But then the lifted geodesic $O(2) g^{t} A \tilde{V}^{\prime}$ on $O(2) \backslash S L_{ \pm}(2, \mathbb{R}) / \tilde{V}^{\prime}$ is asymptotic to the lifted convex core boundary, which is depicted as the boundary between the light and dark gray regions on the right side of Figure 6. But then the coding walk $\left\langle n_{k}\right\rangle$ must grow asymptotically linearly, i.e., there is a $K$ so that for all $k>K$ we have $n_{k+1}=n_{k}+1$ or for all $k>K$ we have $n_{k+1}=n_{k}+1$. But then the growth exponent is $v=1$ which is not allowed in case (L2).

Now we will consider how to obtain non-ergodic covers. We will make use of ideas of Masur and Smillie which first appeared in [MS91, Theorem 2.1]. The criterion developed there for non-ergodicity carries over from the closed surface case to the infinite type case. We will state the (only slightly different) version from [MT02, Theorem 3.3] in our setting. For the following theorem recall that the vertical holonomy of a curve $\gamma$ in a translation surface is the imaginary part of $\int_{\gamma} \alpha$.

Theorem 50 (Masur-Smillie [MS91]). Let $(S, \alpha)$ be a unit area translation surface of possibly infinite topological type, and assume the translation flow is defined for all time almost everywhere. Suppose there is a sequence of directions $\theta_{n}$ tending to the horizontal and a sequence of partitions of the surface into two pieces, $S=A_{n} \sqcup B_{n}$, so that the common boundary consists of a countable union of line segments in direction $\theta_{n}$. Assume further that the absolute values of the vertical holonomies of the segments sum to $h_{n}<\infty$. Suppose also that:

(i) $\lim _{n \rightarrow \infty} h_{n}=0$.

(ii) There are constants $c$ and $c^{\prime}$, so that $0<c<\mu\left(A_{n}\right)<c^{\prime}<1$ for each $n$, where $\mu$ is Lebesgue measure on $\left(S_{L}, \alpha_{L}\right)$.

(iii) $\sum_{n=1}^{\infty} \mu\left(A_{n} \Delta A_{n+1}\right)<\infty$, where $\Delta$ denotes symmetric difference. Then, the translation flow (horizontal straight-line flow) on $\left(S_{L}, \alpha_{L}\right)$ is not ergodic.

The proof as provided in [MT02, Theorem 3.3] works in our setting, so we only explain the main ideas of the proof. Through a measure theoretic argument, one can see that the set $A_{\infty}=\lim \inf A_{n}$ must be almost flow invariant in the sense that for any $t$ the symmetric difference of $A_{\infty}$ with its image under translation flow for time $t$ has Lebesgue measure zero. The set $A_{\infty}$ has measure bounded away from zero and one. An argument involving Fubini's theorem then produces an invariant set which is the same up to sets of Lebesgue measure zero which proves non-ergodicity. The arguments make no use of compactness or any other properties which do not hold in our setting. 
Proof of statement (L3) of Theorem 42. Let $A \in S L(2, \mathbb{R})$ and $h \in \operatorname{Hom}\left(\Gamma, \mathbb{Z}_{2}\right)$ determine a devious cover $A\left(\tilde{S}_{h}, \tilde{\alpha}_{h}\right)$ of $A\left(S_{L}, \alpha_{L}\right)$. We also make several other hypotheses. We consider the coding walk of the geodesic $g^{t} A \tilde{V}^{\prime}$ in $O(2) \backslash S L_{ \pm}(2, \mathbb{R}) / \tilde{V}^{\prime}$, and consider its coding walk $\left\{n_{k}\right\}$. Statement (L3) concerns the case when the coding walk diverges quickly in the sense that the growth exponent $v$ is smaller than $\varphi^{2}$. In light of Remark 44, we can assume that $\lim _{k \rightarrow \infty} n_{k}=-\infty$. By Lemma 43 , we know that $\lim _{m \rightarrow+\infty} \tau_{*}^{m}(h)=\mathbf{0}$.

We will prove that the translation flow on $A\left(\tilde{S}_{h}, \tilde{\alpha}_{h}\right)$ is not ergodic. To do this, we will find a sequence of partitions satisfying the criteria set out by Masur and Smillie (Theorem 50). Our partitions will be obtained by pulling back partitions of the deformed surface $g^{t} A\left(\tilde{S}_{h}, \tilde{\alpha}_{h}\right)$ along a sequence of times tending to $+\infty$.

It will be important for us to label the lifts of the basepoint to our double covers. We label the lifts by the elements of $\mathbb{Z}_{2}$. Then when we deform our surfaces or apply linear maps, we will respect the labels of the basepoint. (Note that all double covers are normal and so admit a translation automorphism swapping the lifts of the basepoint.)

We introduce the following setup. Consider the region $F_{0}$ in $O(2) \backslash S L_{ \pm}(2, \mathbb{R}) / \tilde{V}^{\prime}$. Since the geodesic $g_{t} A V^{\prime}$ is asymptotic to the convex core of $O(2) \backslash S L_{ \pm}(2, \mathbb{R}) / V^{\prime}$, there is a $d>0$ so that this geodesic is contained entirely in the $d$-neighborhood of this convex core. Let $F_{0}^{\prime} \subset F_{0}$ be the closed $d$-neighborhood of the lift of the convex core. Choose an $M_{0} \in S L_{ \pm}(2, \mathbb{R})$ so that $M_{0} \tilde{V}^{\prime}$ lies in $F_{0}^{\prime}$. Now consider another point $B \tilde{V}^{\prime}$ of $F_{0}^{\prime}$. Select a path $\gamma_{B}$ in $F_{0}^{\prime}$ joining $M_{0} \tilde{V}^{\prime}$ to $B \tilde{V}^{\prime}$. We can lift this path in $S L_{ \pm}(2, \mathbb{R}) / \tilde{V}^{\prime}$ to a path in $S L(2, \mathbb{R})$ beginning at $M_{0}$. We let $E(B) \in S L(2, \mathbb{R})$ denote the endpoint of this path. Observe that $E(B)$ lies in the coset $B \tilde{V}^{\prime}$. The selection of $E(B)$ is not quite canonical; it does not depend on the choice of $B$ from the class $B \tilde{V}^{\prime}$, but it depends on the homotopy class of the curve $\gamma_{B}$. Since $F_{0}^{\prime}$ is topologically an annulus, a different choice of path might give us a different $E(B)$, and difference is explained by monodromy around the cusp. Let $\Psi=\left\langle D(\psi)^{2}\right\rangle \subset S L(2, \mathbb{R})$ be the monodromy group of $F_{0}$. We see that while $E(B)$ is not canonical, the coset $E(B) \Psi$ is. We will make choices only depending on $E(B) \Psi$.

Now consider an affine image of our cover, $g^{t} A\left(\tilde{S}_{h}, \tilde{\alpha}_{h}\right)$, and suppose that $g^{t} A \tilde{V}^{\prime}$ lies in the region $F_{n}$. Select an element $R \in V^{\prime}$ which projects to $\tau^{-n} \in V^{\prime} / \tilde{V}^{\prime}$ so that $g^{t} A \tilde{V}^{\prime} R^{-1}=$ $g^{t} A R^{-1} \tilde{V}^{\prime}$ lies in the region $F_{0}^{\prime}$. The action of $R$ on $\operatorname{Hom}\left(\Gamma, \mathbb{Z}_{2}\right)$ is given by $\delta(R)=\tau_{*}^{-n}$. Therefore,

$$
g^{t} A\left(\tilde{S}_{h}, \tilde{\alpha}_{h}\right)=g^{t} A R^{-1}\left(\tilde{S}_{\tau_{*}^{-n}(h)}, \tilde{\alpha}_{\tau_{*}^{-n}(h)}\right),
$$

where equality denotes translation equivalence respecting marked points. Now consider $E\left(g^{t} A R^{-1}\right)$ as in the prior paragraph. We have $E\left(g^{t} A R^{-1}\right)=g^{t} A R^{-1} \tilde{R}^{-1}$ for some $\tilde{R} \in \tilde{V}^{\prime}$. Since $\tilde{V}^{\prime}$ acts trivially on $\operatorname{Hom}\left(\Gamma, \mathbb{Z}_{2}\right)$, we see that

$$
g^{t} A\left(\tilde{S}_{h}, \tilde{\alpha}_{h}\right)=E\left(g^{t} A R^{-1}\right)\left(\tilde{S}_{\tau_{*}^{-n}(h)}, \tilde{\alpha}_{\tau_{*}^{-n}(h)}\right) \quad \text { in } \tilde{\mathcal{O}}_{\mathbb{Z}_{2}}\left(S_{L}, \alpha_{L}\right)
$$

A partition of the cover $\left(\tilde{S}_{\tau_{*}^{-n}(h)}, \tilde{\alpha}_{\tau_{*}^{-n}(h)}\right)$ will then pull back under this translation isomorphism followed by $g^{-t}$ to a partition of our original surface $A\left(\tilde{S}_{h}, \tilde{\alpha}_{h}\right)$. The cover $\left(\tilde{S}_{\tau_{*}^{-n}(h)}, \tilde{\alpha}_{\tau_{*}^{-n}(h)}\right)$ can be thought of two copies of the infinite polygon used to define $\left(S_{L}, \alpha_{L}\right)$ labeled $\mathcal{R}_{0}$ and $\mathcal{R}_{1}$ and glued together according to $\tau_{*}^{-n}(h) \in \operatorname{Hom}\left(\Gamma, \mathbb{Z}_{2}\right)$. The index of the regions is determined by the index of the lift of the basepoint the region contains. We will partition the cover into two subsurfaces $\mathcal{A}$ and $\mathcal{B}$. From our point of view, there are three types of vertical cylinders on the cover $\left(\tilde{S}_{\tau_{*}^{-n}(h)}, \tilde{\alpha}_{\tau_{*}^{-n}(h)}\right)$ : 

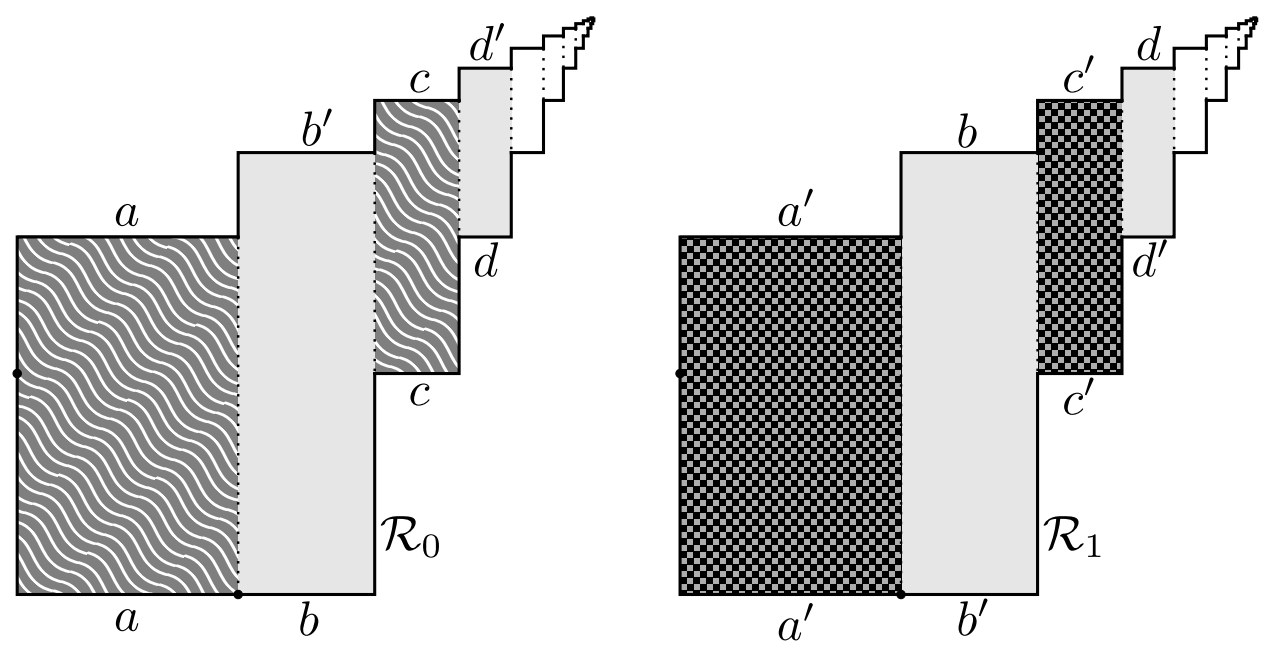

Figure 9. A double cover of $\left(S_{L}, \alpha_{L}\right)$ with some edge gluings of horizontal edges labeled. There are two vertical cylinders of type (1) shown in gray with white waves, two of type (2) with a checkerboard pattern, and two of type (3) shown in light gray.

(1) vertical cylinders that stay entirely in region $\mathcal{R}_{0}$.

(2) vertical cylinders that stay entirely in region $\mathcal{R}_{1}$.

(3) vertical cylinders that pass through both $\mathcal{R}_{0}$ and $\mathcal{R}_{1}$.

Figure 9 illustrates the two regions and the cylinder types. For any integer $j<0$, we have either $\tau_{*}^{-n}(h)(j)=0$ or $\tau_{*}^{-n}(h)(j)=1$. In the first case, we get vertical cylinders of types (1) and (2) passing through lifts of the edge labeled $j$ of the base surface (as depicted in Figure 4), and in the second case, there is a single vertical cylinder of type (3) which passes through both the lifts of edges labeled $j$. We define the surface $\mathcal{A}_{n} \subset\left(\tilde{S}_{\tau_{*}^{-n}(h)}, \tilde{\alpha}_{\tau_{*}^{-n}(h)}\right)$ to be the union of all vertical cylinders of type (1), and we define $\mathcal{B}_{n}$ to be the union of cylinders of the remaining two types. We can push this partition onto the surface $g^{t} A\left(\tilde{S}_{h}, \tilde{\alpha}_{h}\right)$ by applying the affine map $E\left(g^{t} A R^{-1}\right)$ and using the identification given in equation 44. This gives us a partition $\left(\mathcal{A}^{t}, \mathcal{B}^{t}\right)$ of $g^{t} A\left(\tilde{S}_{h}, \tilde{\alpha}_{h}\right)$. Note that while $E\left(g^{t} A R^{-1}\right)$ is not quite canonical as noted in the prior paragraph, it is well defined up to a power of $D(\psi)^{2}$. Note that the partition of $g^{t} A\left(\tilde{S}_{h}, \tilde{\alpha}_{h}\right)$ obtained is the same no mater which element of $E\left(g^{t} A R^{-1}\right) \Psi$ we choose, because the action of $D\left(\psi^{2}\right) \in \tilde{V}^{\prime}$ preserves each vertical cylinder.

For each $t$, we can pullback the partition $\left(\mathcal{A}^{t}, \mathcal{B}^{t}\right)$ of $g^{t} A\left(\tilde{S}_{h}, \tilde{\alpha}_{h}\right)$ to a partition $g^{-t}\left(\mathcal{A}^{t}, \mathcal{B}^{t}\right)$ of $A\left(\tilde{S}_{h}, \tilde{\alpha}_{h}\right)$. This is actually a sequence of partitions, as we now explain. When $g^{t} A \tilde{V}^{\prime}$ lies in the region $F_{n}$, we have that $t$ lies in some interval $\left(t_{k}, t_{k+1}\right)$ where $n_{k}=n$. We claim the partition $g^{-t}\left(\mathcal{A}^{t}, \mathcal{B}^{t}\right)$ is independent of the choice of $t$ from this interval. This is because the coset $g^{-t} E\left(g^{t} A R^{-1}\right) \Psi$ is constant on the interval, since given a path to $g^{t} A R^{-1}$ we can get a path to $g^{t_{*}} A R^{-1}$ by traveling along the geodesic, and this change is canceled by the application of $g^{-t}$. Thus the partition only depends on $k$, and we define $\mathcal{A}_{k}^{\prime}=g^{-t}\left(\mathcal{A}^{t}\right)$ and $\mathcal{B}_{k}^{\prime}=g^{-t}\left(\mathcal{A}^{t}\right)$ for any $t$ satisfying $t_{k}<t<t_{k+1}$. These subsurfaces partition $A\left(\tilde{S}_{h}, \tilde{\alpha}_{h}\right)$.

It remains to check that our sequence of partitions $\left(\mathcal{A}_{k}^{\prime}, \mathcal{B}_{k}^{\prime}\right)$ satisfy the criterion of Masur and Smillie.

Consider statement (i), we need to show the total vertical holonomy of $\partial \mathcal{A}_{k}^{\prime}$ tends to zero as $k \rightarrow \infty$. This can be based on the observation that the total length of the boundary of the 
partitions $\left(\mathcal{A}_{n}, \mathcal{B}_{n}\right)$ of the surface $\left(\tilde{S}_{\tau_{*}^{-n}(h)}, \tilde{\alpha}_{\tau_{*}^{-n}(h)}\right)$ can be bounded from above independent of $n$ and $\tau^{-n}(h)$. Indeed, for any double cover, the total length of all boundaries of all vertical cylinders is a finite constant independent of the cover. It is twice the corresponding constant for the base surface $\left(S_{L}, \alpha_{L}\right)$, and the constant there is finite because the cylinders decay in size exponentially. We can choose $R \in V^{\prime}$ as above so that $g^{t} A R^{-1} \tilde{V}^{\prime}$ lies in the region $F_{0}^{\prime}$ when $t_{k}<t<t_{k+1}$. Select $t$ so that $g^{t} A R^{-1} \tilde{V}^{\prime}$ lies in a fixed compact set of $F_{0}^{\prime}$, which can be taken to be a neighborhood of the convex core with a cusp neighborhood removed. Note then that by definition of $E$, the quantity $E\left(g^{t} A R^{-1}\right) \Psi$ is taken from a compact subset of $S L(2, \mathbb{R}) / \Psi$. So, in particular we get a uniform upper bound $L<\infty$ on the length of the boundary of the partition $\left(\mathcal{A}^{t}, \mathcal{B}^{t}\right)$ of the surface $g^{t} A\left(\tilde{S}_{h}, \tilde{\alpha}_{h}\right)$. (This uses the identification of equation 44.) In particular, the vertical component of this length is bounded from above by $L$. Pulling back via $g^{-t}$, we see that the vertical component of the length of the common boundary of $\mathcal{A}_{k}^{\prime}$ and $\mathcal{B}_{k}^{\prime}$ is bounded from above by $e^{-t} L$, which tends to zero as $k \rightarrow \infty$ because there is a uniform lower bound on $t_{k+1}-t_{k}$. This verifies statement (i).

To prove statement (ii), we need to make use of the assumptions that $\lim _{k \rightarrow \infty} n_{k}=+\infty$ and $\lim _{m \rightarrow \infty} \tau_{*}^{m}(h)=\mathbf{0}$. By Lemma 47, there is an $M \geq 0$ and an integer $j$ so that $m>M$ implies that $P\left(\tau_{*}^{m}(h)\right)=j+m$, where $P$ denotes proximity; see equation 37. Since $\left\{n_{k}\right\}$ tends to $+\infty$, there is a $K$ so that $k>K$ implies $P\left(\tau_{*}^{-n_{k}}(h)\right)>1$. Since the proximity is larger than one, $\tau_{*}^{-n_{k}}(h)(-1)=0$. This means that there are two vertical cylinders in the surface $\left(\tilde{S}_{\tau_{*}^{-n_{k}(h)}}, \tilde{\alpha}_{\tau_{*}^{-n_{k}(h)}}\right)$ passing through lifts of the edge labeled -1 . One of these cylinders lies in $\mathcal{A}_{n_{k}}$ and the other lies in $\mathcal{B}_{n_{k}}$. Thus when $k>K$, we obtain upper and lower bounds on the area of $\mathcal{A}_{n_{k}}$. Since the partition $\left(\mathcal{A}_{k}^{\prime}, \mathcal{B}_{k}^{\prime}\right)$ was obtained by pulling back along an area preserving map, the same bounds hold here. (We only need to verify statement (ii) holds only for all but finitely many $k$.)

In order to understand (iii), we need to investigate how our partition changes when the geodesic $g^{t} A \tilde{V}^{\prime}$ passes from a region $F_{n}$ to $F_{n+1}$. Consider a time $t$ so that $g^{t} A \tilde{V}^{\prime}$ lies in the common boundary between $F_{n}$ and $F_{n+1}$. This is the point at which the partition changes. First consider this point $g^{t} A \tilde{V}^{\prime}$ as part of $F_{n}$. Then, we build a partition $\left(\mathcal{A}_{n}, \mathcal{B}_{n}\right)$ of the cover $\left(\tilde{S}_{\tau_{*}^{-n}(h)}, \tilde{\alpha}_{\tau_{*}^{-n}(h)}\right)$ as described above, and apply $g^{-t} E\left(g^{t} A R_{n}^{-1}\right)$, where $R_{n} \in V^{\prime}$ is any Veech group element carrying $F_{0}$ to $F_{n}$, to partition our original surface. Here $E\left(g^{t} A R_{n}^{-1}\right) \in$ $S L(2, \mathbb{R})$ is determined by lifting a path joining the $M_{0} \tilde{V}^{\prime}$ to $g^{t} A R_{n}^{-1} \tilde{V}^{\prime}$ within $F_{0}^{\prime}$. Now we consider what happens when we consider $g^{t} A \tilde{V}^{\prime}$ as part of $F_{n+1}$. We construct a partition $\left(\mathcal{A}_{n+1}, \mathcal{B}_{n+1}\right)$ of the cover $\left(\tilde{S}_{\tau_{*}^{-n-1}(h)}, \tilde{\alpha}_{\tau_{*}^{-n-1}(h)}\right)$. We apply $g^{-t} E\left(g^{t} A R_{n+1}^{-1}\right)$, where $R_{n+1}$ is some Veech group element carrying $F_{0}$ to $F_{n+1}$, to partition our original surface. We need to understand the area of the symmetric difference of the pulled back partitions. It is equivalent to find the area of the symmetric difference between the partition $\left(\mathcal{A}_{n}, \mathcal{B}_{n}\right)$ and the other partition of $\left(\tilde{S}_{\tau_{*}^{-n}(h)}, \tilde{\alpha}_{\tau_{*}^{-n}(h)}\right)$ obtained as the image of $\left(\mathcal{A}_{n+1}, \mathcal{B}_{n+1}\right)$ under

$$
R_{*}=\left(g^{-t} E\left(g^{t} A R_{n}^{-1}\right)\right)^{-1} g^{-t} E\left(g^{t} A R_{n+1}^{-1}\right)=E\left(g^{t} A R_{n}^{-1}\right)^{-1} E\left(g^{t} A R_{n+1}^{-1}\right) .
$$

The elements $E\left(g^{t} A R_{n}^{-1}\right)$ and $E\left(g^{t} A R_{n+1}^{-1}\right)$ are determined based on lifting paths $\gamma_{n}$ and $\gamma_{n+1}$ in $S L_{ \pm}(2, \mathbb{R}) / \tilde{V}^{\prime}$ to paths $\tilde{\gamma}_{n}$ and $\tilde{\gamma}_{n+1}$ respectively. The value of $R_{*} \in S L(2, \mathbb{R})$ can then be determined by lifting the path obtained by first following $\gamma_{1}$ and then following the translated path $E\left(g^{t} A R_{n}^{-1}\right)\left(\gamma_{2}\right)$ backward. The result is a path which passes once through the common boundary between $F_{0}$ and $F_{1}$, and joins the equivalence class of the $M_{0}$ to $D(\rho \circ \psi) \tilde{V}^{\prime}$. The value of $R_{*}$ is the endpoint of this path lifted to $S L(2, \mathbb{R})$, which we see lies 
in the double coset

$$
\Psi D(\rho \circ \psi) \Psi \in \Psi \backslash S L(2, \mathbb{R}) / \Psi .
$$

As the action of an element $\Psi$ does not change our partitions, we can choose to work with the simplest element from our point of view, $R_{*}=D(\rho \circ \psi)$.

We need to estimate the area of the symmetric difference $\mathcal{A}_{n} \Delta R_{*}\left(\mathcal{A}_{n+1}\right)$. Here, we interpret $R_{*}$ as an affine homeomorphism

$$
R_{*}:\left(\tilde{S}_{\tau_{*}^{-n-1}(h)}, \tilde{\alpha}_{\tau_{*}^{-n-1}(h)}\right) \rightarrow\left(\tilde{S}_{\tau_{*}^{-n}(h)}, \tilde{\alpha}_{\tau_{*}^{-n}(h)}\right)
$$

which is characterized by its derivative and respects the labels of lifts of the basepoint. Observe that the action of the matrix $R_{*}$ carries vertical cylinders to horizontal cylinders and preserves their widths. It also respects the labeling of the lifts of the basepoints. Suppose that the proximity $P\left(\tau_{*}^{-n-1}(h)\right)=p$. For any integer $e<0$ with $-e<p$, we have $\tau_{*}^{-n-1}(h)(e)=0$, and thus the vertical cylinder in $\left(\tilde{S}_{\tau_{*}^{-n-1}(h)}, \tilde{\alpha}_{\tau_{*}^{-n-1}(h)}\right)$ starting in regions $\mathcal{R}_{0}$ and passing through a lift of the edge labeled $e$ in Figure 4 lies in the subsurface $\mathcal{A}_{n+1}$. Consider the union $U \subset \mathcal{A}_{n+1}$ of all such vertical cylinders in $\mathcal{A}_{n+1}$ with $-p<e<0$. We observe that because basepoint labels are preserved, $R_{*}(U) \subset \mathcal{A}_{n}$, with each vertical cylinder through $e$ being sent to a horizontal cylinder in $\mathcal{A}_{n}$ passing through a vertical edge labeled $-e$. Let $\nu$ be the Lebesgue probability measure on $\left(\tilde{S}_{\tau_{*}^{-n}(h)}, \tilde{\alpha}_{\tau_{*}^{-n}(h)}\right)$. We get the upper bound on the area of the symmetric difference

$$
\nu\left(\mathcal{A}_{n} \Delta R_{*}\left(\mathcal{A}_{n+1}\right)\right) \leq \nu\left(\mathcal{A}_{n}\right)+\nu\left(R_{*}\left(\mathcal{A}_{n+1}\right)\right)-2 \nu(U) \leq 1-2 \nu(U) .
$$

Observe that successively smaller cylinders in $\left(S_{L}, \alpha_{L}\right)$ decrease in area by a factor of $\varphi^{2}$. It follows that there is a constant $\alpha>0$ so that $1-2 \nu(U)<\alpha \varphi^{-2 p}$. So, we see

$$
\nu\left(\mathcal{A}_{n} \Delta R_{*}\left(\mathcal{A}_{n+1}\right)\right)<\alpha \varphi^{-2 p},
$$

where $p=P\left(\tau_{*}^{-n-1}(h)\right)$ as above.

Let $\mu$ be the normalized Lebesgue measure on the surface $A\left(\tilde{S}_{h}, \tilde{\alpha}_{h}\right)$. The prior paragraph gives an upper bound on $\mu\left(\mathcal{A}_{k}^{\prime} \Delta \mathcal{A}_{k+1}^{\prime}\right)$ when $n_{k+1}=n_{k}+1$ in terms of the proximity $p\left(n_{k+1}\right)=P\left(\tau_{*}^{-n_{k}-1}(h)\right)$. A similar bound holds for the case when $n_{k+1}=n_{k}-1$; there is a constant $\alpha^{\prime}>\alpha$ so that

$$
\mu\left(\mathcal{A}_{k}^{\prime} \Delta \mathcal{A}_{k+1}^{\prime}\right)<\alpha^{\prime} \varphi^{-2 p\left(n_{k+1}\right)}
$$

regardless if $n_{k+1}$ equals $n_{k}+1$ or $n_{k}-1$.

Now we will consider the total sum of the symmetric differences to verify statement (iii). Let $V_{N}=\#\left\{k: n_{k}=N\right\}$ for integers $N$. By assumption, each $V_{N}$ is finite, and $V_{N}=0$ for $N<N_{0}$ for some $N_{0} \in \mathbb{Z}$. Then,

$$
\sum_{k} \mu\left(\mathcal{A}_{k}^{\prime} \Delta \mathcal{A}_{k+1}^{\prime}\right) \leq \sum_{N=N_{0}}^{\infty} V_{N} \varphi^{-2 p(N)} .
$$

Now we incorporate the assumption that $\lim _{N \rightarrow+\infty} \tau_{*}^{N}(h)=\mathbf{0}$. From Lemma 47, we obtain an $M \geq 0$ and an integer $j$ so that $N>M$ implies that $P\left(\tau_{*}^{N}(h)\right)=j+N$. Let $X<\infty$ be the sum over the terms with $N \leq M$, then we see that

$$
\sum_{k} \mu\left(\mathcal{A}_{k}^{\prime} \Delta \mathcal{A}_{k+1}^{\prime}\right) \leq X+\sum_{N=M+1}^{\infty} V_{N} \varphi^{-2(j+N)} .
$$


An application of the root test tells us this sum converges if $\lim _{\sup } \operatorname{su}_{N \rightarrow \infty} V_{N}^{1 / N}<\varphi^{2}$. Since this was a hypothesis, we have verified statement (iii). As an application of Theorem 50 , we see that the translation flow on $A\left(\tilde{S}_{h}, \tilde{\alpha}_{h}\right)$ is not ergodic.

\section{REFERENCES}

[BM74] Alan F. Beardon and Bernard Maskit, Limit points of Kleinian groups and finite sided fundamental polyhedra, Acta Math. 132 (1974), 1-12. MR 0333164 (48 \#11489)

[Bow13] Joshua P. Bowman, The complete family of Arnoux-Yoccoz surfaces, Geometriae Dedicata 164 (2013), no. 1, 113-130 (English).

[Buf13] Aleksandr Igorevich Bufetov, Limit theorems for suspension flows over vershik automorphisms, Russian Mathematical Surveys 68 (2013), no. 5, 789-860.

[CE07] Yitwah Cheung and Alex Eskin, Unique ergodicity of translation flows., Forni, Giovanni (ed.) et al., Partially hyperbolic dynamics, laminations, and Teichmüller flow. Selected papers of the workshop, Toronto, Ontario, Canada, January 2006. Providence, RI: American Mathematical Society (AMS); Toronto: The Fields Institute for Research in Mathematical Sciences. Fields Institute Communications 51, 213-221 (2007)., 2007.

[CGL06] R. Chamanara, F. P. Gardiner, and N. Lakic, A hyperelliptic realization of the horseshoe and baker maps, Ergodic Theory Dynam. Systems 26 (2006), no. 6, 1749-1768. MR 2279264 (2008j:37088)

[Cha04] R. Chamanara, Affine automorphism groups of surfaces of infinite type, In the tradition of Ahlfors and Bers, III, Contemp. Math., vol. 355, Amer. Math. Soc., Providence, RI, 2004, pp. 123-145. MR 2145060 (2006b:30077)

[DEDML98] Mirko Degli Esposti, Gianluigi Del Magno, and Marco Lenci, An infinite step billiard, Nonlinearity 11 (1998), no. 4, 991-1013. MR 1632594 (99i:58092)

[Hoo13] W. Patrick Hooper, Immersions and the space of all translation structures, preprint, arXiv:1310.5193, 2013.

[Hoo15] W. Patrick Hooper, The invariant measures of some infinite interval exchange maps, Geom. Topol. 19 (2015), no. 4, 1895-2038. MR 3375521

[Hoo16] W. Patrick Hooper, Immersions and translation structures $i$ : The space of structures on the pointed disk, 2016, preprint, arXiv:1309.4795.

[HR16] F. Herrlich and A. Randecker, Notes on the Veech group of the Chamanara surface, ArXiv e-prints (2016).

[Hub06] John Hamal Hubbard, Teichmüller theory and applications to geometry, topology, and dynamics. Vol. 1, Matrix Editions, Ithaca, NY, 2006, Teichmüller theory, With contributions by Adrien Douady, William Dunbar, Roland Roeder, Sylvain Bonnot, David Brown, Allen Hatcher, Chris Hruska and Sudeb Mitra, With forewords by William Thurston and Clifford Earle. MR 2245223

[HW13] Pascal Hubert and Barak Weiss, Ergodicity for infinite periodic translation surfaces, Compos. Math. 149 (2013), no. 8, 1364-1380 (English).

[LTn16] Kathryn Lindsey and Rodrigo Treviño, Infinite type flat surface models of ergodic systems, Discrete Contin. Dyn. Syst. 36 (2016), no. 10, 5509-5553. MR 3543559

[Mas92] Howard Masur, Hausdorff dimension of the set of nonergodic foliations of a quadratic differential., Duke Math. J. 66 (1992), no. 3, 387-442 (English), Contains Masur's Criterion for ergodicity.

[MS91] Howard Masur and John Smillie, Hausdorff dimension of sets of nonergodic measured foliations, Ann. of Math. (2) 134 (1991), no. 3, 455-543. MR 1135877 (92j:58081)

[MT02] Howard Masur and Serge Tabachnikov, Rational billiards and flat structures, Handbook of dynamical systems, Vol. 1A, North-Holland, Amsterdam, 2002, pp. 1015-1089. MR 1928530 (2003j:37002)

[PSV11] Piotr Przytycki, Gabriela Schmithüsen, and Ferrán Valdez, Veech groups of Loch Ness monsters., Ann. Inst. Fourier 61 (2011), no. 2, 673-687 (English).

[Pud13] Doron Puder, Primitive words, free factors and measure preservation, Israel Journal of Mathematics (2013), 1-49 (English). 
[Rad25] Tibor Radó, Über den begriff der riemannschen fläche, Acta Litt. Sci. Szeged 2 (1925), no. 101$121,10$.

[Ran14] Anja Randecker, Wild translation surfaces and infinite genus, arXiv:1410.1501, 2014.

[Ric63] Ian Richards, On the classification of noncompact surfaces, Transactions of the American Mathematical Society 106 (1963), no. 2, 259-269.

[Rya97] Kay Ryan, Elephant rocks, Grove Press, 1997.

[Thu88] William P. Thurston, On the geometry and dynamics of diffeomorphisms of surfaces, Bull. Amer. Math. Soc. (N.S.) 19 (1988), no. 2, 417-431. MR 956596 (89k:57023)

[Tre14] Rodrigo Treviño, On the ergodicity of flat surfaces of finite area, Geometric and Functional Analysis (2014), 1-27 (English).

[Tre16] _ Flat surfaces, Bratteli diagrams, and unique ergodicity à la Masur, to appear in the Israel Journal of Mathematics, arXiv:1604.03572, 2016.

[Tro99] Serge Troubetzkoy, Billiards in infinite polygons, Nonlinearity 12 (1999), no. 3, 513-524. MR 1690190 (2000b:37040)

[Vee89] W. A. Veech, Teichmüller curves in moduli space, Eisenstein series and an application to triangular billiards, Invent. Math. 97 (1989), no. 3, 553-583. MR 1005006 (91h:58083a)

[VJ68] D. Vere-Jones, Ergodic properties of nonnegative matrices. II, Pacific J. Math. 26 (1968), 601620. MR 0236745 (38 \#5039)

The City College of New York, New York, NY, USA 10031

CUNy Graduate Center, New York, NY, USA 10016

E-mail address: whooper@ccny.cuny.edu

Department of Mathematics, University of Maryland, College Park

E-mail address: rodrigo@math.umd.edu 